\title{
Multi-frequency study of the B3-VLA sample ${ }^{\star}$
}

\author{
II. The database \\ M. Vigotti ${ }^{1}$, L. Gregorini ${ }^{1,2}$, U. Klein ${ }^{3}$, and K.-H. Mack ${ }^{1,3}$ \\ 1 Istituto di Radioastronomia del CNR, Via Gobetti 101, I-40129 Bologna, Italy \\ 2 Dipartimento di Fisica, Università di Bologna, Via Irnerio 46, I-40126 Bologna, Italy \\ 3 Radioastronomisches Institut der Universität Bonn, Auf dem Hügel 71, D-53121 Bonn, Germany
}

Received June 29; accepted July 19, 1999

\begin{abstract}
We present total flux densities of 1049 radio sources in the frequency range from $151 \mathrm{MHz}$ to $10.6 \mathrm{GHz}$. These sources belong to the B3-VLA sample, which is complete down to $100 \mathrm{mJy}$ at $408 \mathrm{MHz}$. The data constitute a homogeneous spectral database for a large sample of radio sources, 50 times fainter than the $3 \mathrm{C}$ catalogue, and will be used to perform a spectral ageing analysis, which is one of the critical points in understanding the physics and evolution of extragalactic radio sources.
\end{abstract}

Key words: surveys - radio continuum: general

\section{Introduction}

Homogeneous databases over a wide frequency range for a large sample of radio sources with intermediate or low flux densities are an important ingredient to modern astrophysics. We have therefore embarked on a project to obtain flux densities for the B3-VLA sample (Vigotti et al. 1989) over a frequency range as wide as possible. The aim is to study the spectral properties of a complete sample of radio sources.

The B3-VLA sample is composed of sources that are roughly equally distributed in five flux density intervals, i.e. 50 times fainter than the 3C survey (Bennett 1962). The sample now contains 1049 radio sources, instead of 1050 listed in the previous papers dealing with the B3-VLA sample: the source $2302+396$ which was already indicated as a possible spurious source close to a grating ring in the B3 catalogue (Ficarra et al. 1985) was deleted from the list since it was recognized as a CLEAN artifact. In fact it could not be found neither in the WENSS nor in the NVSS catalogue.

Send offprint requests to: M. Vigotti (vigotti@ira.bo.cnr.it)

* Table 2 is also available in electronic form at the CDS via anonymous ftp to cdsarc.u-strasbg.fr (130.79.128.5) or via http://cdsweb.u-strasbg.fr/Abstract.html
This paper is the second of a series describing the multifrequency properties of the B3-VLA sample. In the first paper we presented the radio continuum data at $10.6 \mathrm{GHz}$ obtained with the Effelsberg radio telescope (Gregorini et al. 1998, hereafter Paper I). We detected $99 \%$ of the radio sources, with a typical flux density error of about $1 \mathrm{mJy}$ for the fainter ones.

Here we present the spectral database of the whole sample consisting of flux densities at $151 \mathrm{MHz}, 327 \mathrm{MHz}$, $408 \mathrm{MHz}, 1.4 \mathrm{GHz}, 4.85 \mathrm{GHz}$, and $10.6 \mathrm{GHz}$. Additional observations were performed for 478 sources at $4.85 \mathrm{GHz}$, which were necessary to complete the information at this frequency and to measure also at $4.85 \mathrm{GHz}$ the polarization detected at $10.6 \mathrm{GHz}$.

Section 2 describes the observations and data reduction at $4.85 \mathrm{GHz}$. In Sect. 3 we present the database, with an accurate description of the method used to obtain the flux densities and the errors at each frequency. In Sect. 4 the data table is presented with a discussion of the data quality.

\section{Observations at $4.85 \mathrm{GHz}$}

The observations reported here have been carried out between July 1994 and March 1999. Until August 1995 the old $\lambda 6$-cm correlation receiver system was employed. This system had two feeds in the secondary focus of the 100-m telescope. The right-hand circular polarization outputs from each feed (obtained after the polarizers in the waveguides) were correlated via a 3-dB hybrid to yield a differential total-power signal of the two feeds. This double-beam ensured minimal atmospheric disturbance to the signal. Amplification in the first stage was achieved with cooled FET's. The main horn was connected to an IF-polarimeter to deliver the Stokes $U$ and $Q$ parameters for full linear polarization information. The system operated at a centre frequency of $4.75 \mathrm{GHz}$, with a 
Table 1. Measured flux densities of B3-VLA sources

\begin{tabular}{rlr}
\hline Frequency & Reference & $\%$ \\
\hline $151 \mathrm{MHz}$ & Hales et al. (1988) & 89 \\
$327 \mathrm{MHz}$ & Rengelink et al. (1997) & 100 \\
$408 \mathrm{MHz}$ & Ficarra et al. (1985) & 100 \\
$1.4 \mathrm{GHz}$ & Condon et al. (1998) & 100 \\
$4.85 \mathrm{GHz}$ & present paper & 100 \\
& Kulkarni et al. (1990) & 60 \\
& Gregory et al. (1996) & 83 \\
$10.6 \mathrm{GHz}$ & Gregorini et al. (1998) & 99 \\
\hline
\end{tabular}

bandwidth of $500 \mathrm{MHz}$. The receiver system temperature was $\sim 70 \mathrm{~K}$ on the sky (zenith, clear sky).

In August 1995 these receivers were replaced by two stable total-power systems, with HEMT amplifiers in the first stage. Here the differential signal is retrieved by subtracting the calibrated signals in the computer. Each of the two total-power receivers is connected to an IFpolarimeter. This system operates at $4.85 \mathrm{GHz}$, the bandwidth is $500 \mathrm{MHz}$. The receiver system temperature has been greatly improved to $30 \mathrm{~K}$ on the sky (zenith, clear sky).

The half-power beam width was $147^{\prime \prime}$ for the old and $143^{\prime \prime}$ for the new receiver system and the beam throw was 8.2 in both cases. The sources were observed by crossscanning the telescope in right ascension and declination, with a scan length of $15^{\prime}$. The scanning speed was $30^{\prime} / \mathrm{min}$, and the total number of scans was adjusted to the expected flux density of each source. Sources with angular extents significantly exceeding the beam size or exhibiting significant confusion in the cross-scans were mapped in the double-beam mode and subsequently restored to the equivalent single-beam images using the restoration algorithm of Emerson et al. (1979). The scan separation was $1^{\prime}$, and the map sizes adjusted such as to account for the source size and the beam separation. The total number of sources mapped this way is 6 .

Telescope pointing, focussing and polarimeter adjustments were regularly checked by cross-scanning the point sources NGC 7027, 3C 48, 3C 84, 3C 138, 3C 147, 3C 196, 3C 286 and $3 \mathrm{C} 295$. The latter two sources served also as flux density calibrators.

\section{Database}

In Table 1 we present the information available for the B3-VLA sample. Columns 1 and 2 list the frequency and reference to the relevant paper, Col. 3 gives the percentage of sources for which the data are available.

\section{1. $151 \mathrm{MHz}$ data}

These flux densities were obtained by cross-correlating the 6C survey (Hales et al. 1988) with the B3-VLA sample. The search radius used was $100^{\prime \prime}$, which corresponds to a combined $3-\sigma$ error for the fainter sources. We do not expect any chance coincidences, owing to the low source density at $151 \mathrm{MHz}$ (4.1 sources per square degree). The values quoted in Table 2 are the peak flux densities for sources with an angular extent $<100^{\prime \prime}$ (extents taken from Vigotti et al. 1989), and the integrated ones (listed in the $6 \mathrm{C}$, Hales et al. 1988) for larger sources. The error in the same table is computed as a constant term of $40 \mathrm{mJy}$, plus a $5 \%$ contribution due to the uncertainty of the flux density scale.

Since these data are on the flux scale of Roger et al. (1973, RBC), we used the spectral indices reported by these authors to calculate the flux density of their calibrator sources at $178 \mathrm{MHz}$. In this way we could compare the scale of Roger et al. (1973) with the one of Kellermann et al. $(1969, \mathrm{KPW})$. The ratio between these two flux density scales is $\mathrm{KPW} / \mathrm{RBC}=0.96$. Baars et al. (1977, $\mathrm{BGPW}$ ) report a ratio of $\mathrm{BGPW} / \mathrm{KPW}=1.051$. Thus, the ratio $\mathrm{BGPW} / \mathrm{RBC}$ turns out to be 1.008; therefore no correction was applied at $151 \mathrm{MHz}$.

\section{2. $327 \mathrm{MHz}$ data}

For sources with an angular extent $<50^{\prime \prime}$ we crosscorrelated the B3-VLA positions with the WENSS source list (Rengelink et al. 1997) using a window of $11^{\prime \prime}$ in right ascension and $22^{\prime \prime}$ in declination. For the more extended ones we used a window of $40^{\prime \prime}$ in right ascension and $80^{\prime \prime}$ in declination. The total area searched was 0.03 square degrees. The WENSS source density is about 21.3 per square degree so that the contamination by chance coincidences is negligible. For the flux density errors we used the formula given by Rengelink et al. (1997), with a noise contribution of $4.5 \mathrm{mJy}$ (which is the average value in the B3-VLA area), plus $4 \%$ due to the calibration uncertainty $\Delta_{\text {cal }}$.

Sources with a complex structure (as marked in the WENSS catalogue; Rengelink et al. 1997) were inspected directly on the WENSS maps, and their flux densities computed with the AIPS task TVSTAT. For these sources the errors $\Delta S$ were computed as follows:

$\Delta S=\sqrt{\left(\Delta_{\mathrm{cal}} \cdot S\right)^{2}+\sigma_{\mathrm{l}}^{2} \cdot \frac{A_{\mathrm{s}}}{A_{\mathrm{b}}}}$

Here $\sigma_{\mathrm{l}}$ is the local noise in the map, $A_{\mathrm{s}}$ the area covered by the radio source, and $A_{\mathrm{b}}$ is the beam area. The flux densities in the WENSS survey are on the scale of Baars et al. (1977).

\section{3. $408 \mathrm{MHz}$ data}

The flux densities were taken from the B3 survey, except for extended sources for which an integrated flux density was used (Vigotti et al. 1989). For the computation of the errors we used $35 \mathrm{mJy}$ as the constant term and $3 \%$ for the term proportional to the source flux density (Ficarra et al. 1985). The flux density scale of these data is based on 3C 123, and agrees with the scale of Baars et al. (1977) to within $2 \%$. Therefore, no correction was applied. 


\section{4. $1.4 \mathrm{GHz}$ data}

The flux densities were computed from the maps of the NRAO VLA SKY Survey (NVSS, Condon et al. 1998), centred on the B3-VLA positions using an automatic twocomponent Gaussian fit algorithm similar to the AIPS task JMFIT. For the unresolved sources the difference between our flux density and that listed in the NVSS catalogue is negligible $(<2 \%)$. The errors were calculated with the formula of Condon et al. (1998), where the noise and confusion term is $0.45 \mathrm{mJy} /$ beam and the calibration uncertainty is $3 \%$.

For the extended and complex sources the flux densities were computed using the AIPS task TVSTAT. Their errors were computed as above (Sect. 3.2). The flux densities are on the scale of Baars et al. (1977).

\subsection{85 GHz data}

All sources not available in the literature (Kulkarni et al. 1990; Gregory et al. 1996) have been observed as described in Sect. 2. The flux densities of Kulkarni et al. (1990), and those observed by us before August 1995 were shifted from $4.75 \mathrm{GHz}$ to $4.85 \mathrm{GHz}$ using the spectral index of the radio source. For the flux densities presented in this paper we adopted $1.0 \mathrm{mJy}$ as the noise contribution, and $2 \%$ as the contribution proportional to the flux density. Another $0.45 \mathrm{mJy}$ is added to account for source confusion (Reich 1993). For the data of Kulkarni et al. (1990) the errors are $2 \mathrm{mJy}$ and $2 \%$, respectively. The errors of the flux densities taken from Gregory et al. (1996) are listed in the GB6 catalogue. In 2 cases, $1412+397$ and $2341+396 \mathrm{~B}$, the sources could not be separated from a closeby confusing source. We used the flux densities from our measurements $(45.8 \%)$. In cases where those were not available the flux densities reported by Kulkarni et al. $(1990 ; 42.4 \%)$ or Gregory et al. $(1996 ; 11.8 \%)$ were taken.

The GB6 maps of the sources with extension larger than $70^{\prime \prime}$ were downloaded using SkyView. In addition, the most extended ones $(0136+396,0157+405 \mathrm{~A}, 0248+467$, $0703+426 \mathrm{~A}, 1141+374$, and $1309+412 \mathrm{~A}$ ) were mapped in Effelsberg. In all cases the flux densities were determined with AIPS task TVSTAT and the errors were calculated as described above (Sect. 3.2). The flux densities are on the scale of Baars et al. (1977).

\section{6. $10.6 \mathrm{GHz}$ data}

In Table 2 we list the integrated flux densities as well as the errors computed using the formula presented in Paper I. Here, the noise term is $0.8 \mathrm{mJy}$, confusion contributes $0.08 \mathrm{mJy}$, and the term proportional to the flux is $2 \%$. The flux densities are on the scale of Baars et al. (1977).

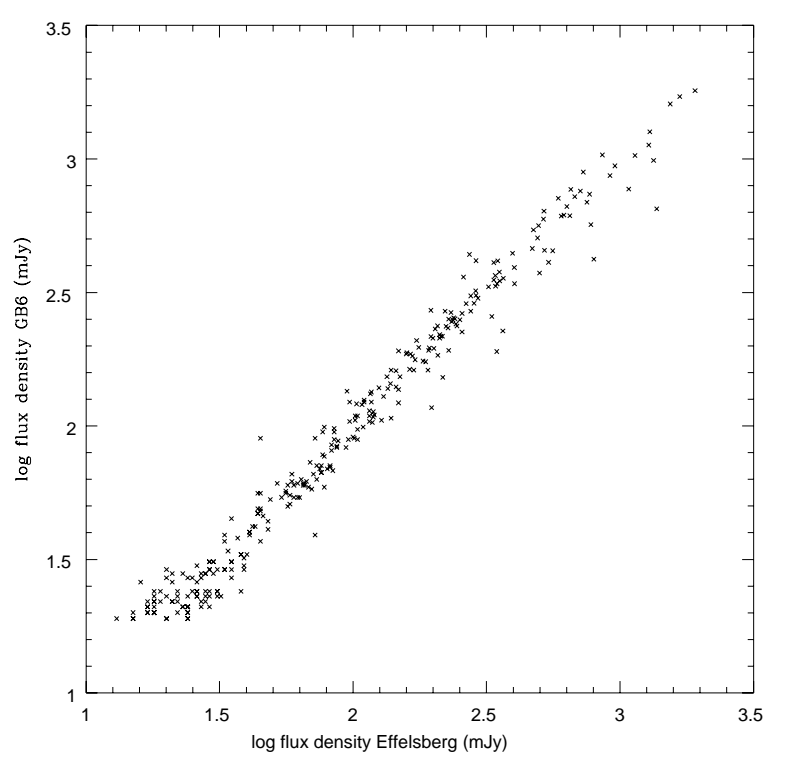

Fig. 1. Flux densities of the GB6 survey versus our measurement

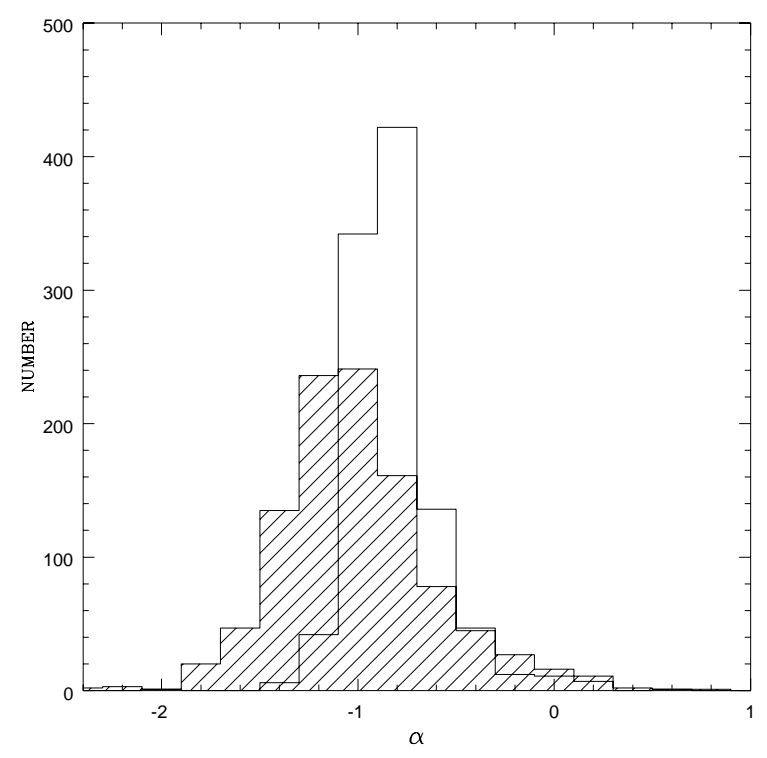

Fig. 2. Histogram of low $\left(\alpha_{1}\right)$ - and high-frequency $\left(\alpha_{\mathrm{h}}\right)$ spectral indices. The blank area corresponds to $\alpha_{1}$, the hashed one to $\alpha_{\mathrm{h}}$

\section{Discussion}

Table 2 presents the whole database. Column 1 lists the B3-VLA name, Cols. 2 and 3 the radio centroid (equinox J2000.0) from Vigotti et al. (1989; computed as the geometric mean of the source components). The following 12 columns list the flux densities and errors at $151 \mathrm{MHz}$, $327 \mathrm{MHz}, 408 \mathrm{MHz}, 1.4 \mathrm{GHz}, 4.85 \mathrm{GHz}$ and $10.6 \mathrm{GHz}$, respectively (all in mJy). The last column contains the sources' optical identifications, abbreviated as follows: g: radio galaxy identified on the POSS-I, most of which are $z \leq 0.5$; G: far radio galaxy with measured redshift 


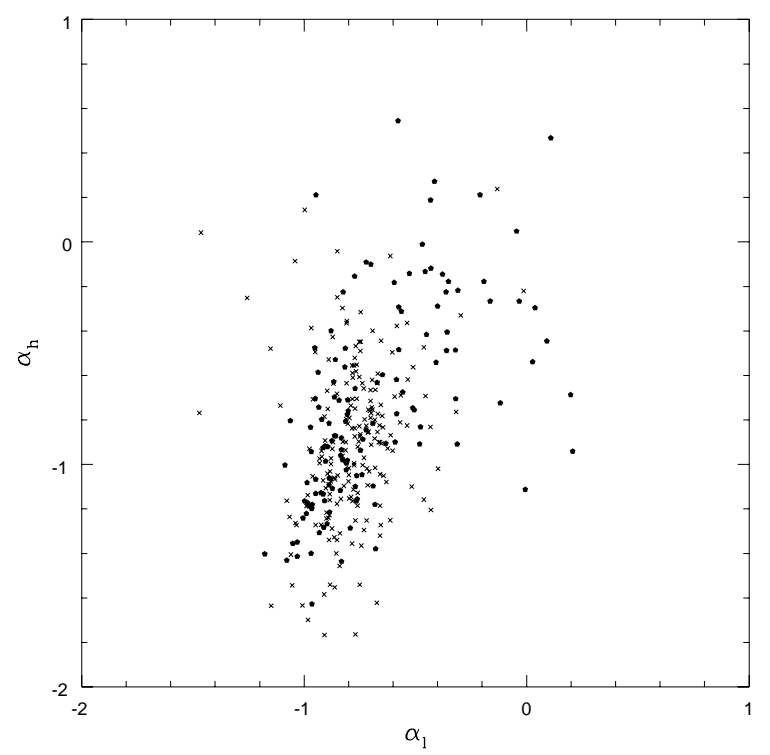

Fig. 3. Colour-colour diagram of B3VLA sources. Galaxies are symbolized by crosses, quasars by dots

$(0.5 \leq z \leq 3.5)$; Q: spectroscopically confirmed quasar; b: blue objects (i.e. non-confirmed quasars); BL: BL Lac; F: featureless spectrum; a blank means "empty field", i.e. it lacks any optical counterpart down to the POSS-I limit (more that $90 \%$ are distant radio galaxies, the remaining ones being quasars with magnitudes fainter than the POSS-I).

For 19 sources the $408 \mathrm{MHz}$ data are not reported. In 15 cases the flux density is affected by a nearby strong source. In four cases the B3-VLA sources were not resolved by the $408 \mathrm{MHz}$ beam.

In order to complete the spectral database we observed 164 sources at $4.85 \mathrm{GHz}$ whose flux densities were not available in the catalogues listed in Table 1; 314 sources with detected polarization at $10.6 \mathrm{GHz}$ were re-observed at $4.85 \mathrm{GHz}$ for future polarization studies. An analysis of the polarization data will be published in a forthcoming paper. In Fig. 1 we show the plot of our measurements versus the GB6 flux densities. Intrinsic source variability is likely to increase the scatter of the plot.

For each source we computed two spectral indices: a low-frequency index $\alpha_{1}(0.3-1.4 \mathrm{GHz})$ and a highfrequency one, $\alpha_{\mathrm{h}}(4.8-10.6 \mathrm{GHz})$. Figure 2 shows the histograms of $\alpha_{\mathrm{l}}$ and $\alpha_{\mathrm{h}}$ (shaded) of 1034 sources, for which four flux densities are available. The resulting median values for the two distributions are $\left\langle\alpha_{1}\right\rangle=-0.853$ and $\left\langle\alpha_{\mathrm{h}}\right\rangle=-1.053\left(S \propto \nu^{\alpha}\right)$.

In Fig. 3 we show a radio colour-colour diagram illustrating the different population areas of radio galaxies and quasars. As already evident in Fig. 2, $\alpha_{\mathrm{h}}$ covers a wider range of values (dispersion 0.40 ) than $\alpha_{1}$ (dispersion 0.23 ). This is to be expected if spectral steepening due to synchrotron and inverse Compton energy losses is important: it changes $\alpha_{\mathrm{h}}$ first, before the sources have aged sufficiently such as to affect $\alpha_{1}$ as well. The corresponding evolutionary track in the $\alpha_{1}-\alpha_{\mathrm{h}}$ diagramme is that populated by the radio galaxies in Fig. 3: if these sources commence their lives with flat (injection) spectra, they should gradually move downward at a faster rate than leftward. Also evident in Fig. 3 is that radio galaxies (crosses) have on average steeper high-frequency spectra than quasars; in particular, the radio galaxies dominate the lowest part of the diagramme.

Some sources (essentially quasars) exhibit extreme values, especially those with flat $\alpha_{1}$ and/or flat $\alpha_{\mathrm{h}}$ (populating the upper and right-hand portion of the plot). These may possess self-absorbed components that become visible in different frequency regimes, depending on their optical thickness. A thorough analysis and interpretation of our results will be presented in a forthcoming paper.

Acknowledgements. We thank Helge Rottmann for his help during the Effelsberg observations. We are grateful to Dr. Heinz Andernach whose comments on the manuscript helped to improve the paper significantly. Part of this work was supported by the Deutsche Forschungsgemeinschaft, grant KL533/4-2, and by the European Commission, TMR Programme, Research Network Contract ERBFMRXCT970034 "CERES". We thank the Italian Ministry for University and Scientic Research (MURST) for partial financial support (grant Cofin98-02-32). We acknowledge the use NASA's SkyView facility (http://skyview.gsfc.nasa.gov) located at NASA Goddard Space Flight Center.

\section{References}

Baars J.W.M., Genzel R., Pauliny-Toth I.I.K., Witzel A., 1977, A\&A 61, 99

Bennett A.S., 1962, Mem. R. Astr. Soc. 68, 163

Condon J.J., Cotton W.D., Greisen E.W., Yin Q.F., Perley R.A., Taylor G.B., Broderick J.J., 1998, AJ 115, 1693

Emerson D.T., Klein U., Haslam C.G.T., 1979, A\&A 76, 92

Ficarra A., Grueff G., Tomassetti G., 1985, A\&AS 59, 255

Gregory P.C., Scott W.K., Douglas K., Condon J.J., 1996, ApJS 103, 427

Gregorini L., Vigotti M., Mack K.-H., Zönnchen J., Klein U., 1998, A\&AS 133, 129 (Paper I)

Hales S.E.G., Baldwin J.E., Warner P.J., 1988, MNRAS 234, 919

Kellermann K.L., Pauliny-Toth I.I.K., Williams P.J.S., 1969, ApJ 157, 1

Kulkarni V.K., Mantovani F., Pauliny-Toth I.I.K., 1990, A\&AS 82,41

Reich W., 1993, in: Effelsberg News 2, MPIfR Bonn

Rengelink R., Tang Y., de Bruyn A.G., Miley G.K., Bremer M.N., Röttgering H.J.A., Bremer M.A.R., 1997, A\&AS 124,259

Roger R.S., Bridle A.H., Costain C.H., 1973, AJ 78, 1030

Vigotti M., Grueff G., Perley R., Clark B.G., Bridle A.J., 1989, AJ 98, 419 98, 419 
Table 2. B3 VLA flux densities

\begin{tabular}{|c|c|c|c|c|c|c|c|c|c|c|c|c|c|c|c|}
\hline B3 name & $\begin{array}{l}\mathrm{RA}(\mathrm{J} 2000) \\
{\left[\begin{array}{ccc}\mathrm{h} & \mathrm{m} & \mathrm{s}\end{array}\right]} \\
\end{array}$ & 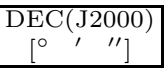 & $\begin{array}{r}S_{151} \\
{[\mathrm{mJy}]}\end{array}$ & $\begin{array}{r}\Delta S_{151} \\
{[\mathrm{mJy}]}\end{array}$ & $\begin{array}{r}S_{327} \\
{[\mathrm{mJy}]}\end{array}$ & $\begin{array}{r}\Delta S_{327} \\
{[\mathrm{mJy}]}\end{array}$ & $\begin{array}{r}S_{408} \\
{[\mathrm{mJy}]}\end{array}$ & $\begin{array}{r}S_{408} \\
{[\mathrm{mJy}]}\end{array}$ & $\begin{array}{l}S_{1400} \\
{[\mathrm{mJy}]}\end{array}$ & $\begin{array}{r}\Delta S_{1400} \\
{[\mathrm{mJy}]} \\
\end{array}$ & $\begin{array}{l}S_{4850} \\
{[\mathrm{mJy}]}\end{array}$ & $\begin{array}{r}\Delta S_{4850} \\
{[\mathrm{mJy}]} \\
\end{array}$ & $\begin{array}{r}\mathrm{c} S_{10550} \\
{[\mathrm{mJy}]}\end{array}$ & $\begin{array}{r}\Delta S_{10550} \\
{[\mathrm{mJy}]}\end{array}$ & Id. \\
\hline $0000+394$ & $\begin{array}{|lll|}00 & 02 & 42.4\end{array}$ & 394634 & 360 & 44 & 270 & 12 & 190 & 31 & 93 & 5 & 36 & 7 & 18 & 1 & \\
\hline $0000+399$ & 000332.0 & 401326 & & & 140 & 7 & 150 & 30 & 53 & 2 & 17 & 1 & 13 & 1 & \\
\hline $0001+395$ & $0004 \quad 15.9$ & 394952 & 530 & 48 & 200 & 9 & 130 & 30 & 33 & 1 & 6 & 1 & 2 & 1 & \\
\hline $0001+398$ & 000419.5 & 400609 & 390 & 45 & 220 & 10 & 220 & 31 & 76 & 2 & 30 & 4 & 11 & 1 & $\mathrm{~g}$ \\
\hline $0003+380$ & 000557.1 & $\begin{array}{lll}38 & 20 & 15\end{array}$ & 790 & 56 & 580 & 24 & 490 & 33 & 568 & 17 & 778 & 16 & 655 & 13 & $\mathrm{~g}$ \\
\hline $0003+387$ & 000620.8 & 390027 & 2910 & 151 & 1750 & 70 & 1360 & 51 & 488 & 15 & 107 & 2 & 30 & 1 & G \\
\hline $0004+380 \mathrm{~A}$ & $00 \quad 0636.4$ & $\begin{array}{llll}38 & 19 & 07\end{array}$ & 1450 & 83 & 980 & 39 & 790 & 38 & 284 & 9 & 83 & 2 & 27 & 1 & \\
\hline $0005+383 \mathrm{~B}$ & 000822.8 & $\begin{array}{llll}38 & 37 & 12\end{array}$ & 970 & 63 & 610 & 25 & 530 & 34 & 315 & 9 & 159 & 3 & 92 & 2 & $\mathrm{~g}$ \\
\hline $0006+397$ & $\begin{array}{llll}00 & 09 & 04.2\end{array}$ & 400146 & 1900 & 103 & 1290 & 52 & 1150 & 46 & 559 & 17 & 370 & 7 & 253 & 5 & $\mathrm{Q}$ \\
\hline $0008+392$ & $\begin{array}{llll}00 & 10 & 37.4\end{array}$ & $3934 \quad 15$ & 500 & 47 & 300 & 13 & 200 & 31 & 75 & 2 & 26 & 1 & 15 & 1 & \\
\hline $0010+392$ & $00 \quad 12 \quad 43.6$ & 393256 & 1090 & 68 & 610 & 25 & 440 & 33 & 151 & 5 & 42 & 1 & 19 & 1 & \\
\hline $0010+395$ & 001312.5 & 394807 & & & 110 & 21 & 140 & 30 & 25 & 1 & 6 & 1 & 3 & 1 & \\
\hline $0010+402$ & 001253.1 & 403248 & 2040 & 110 & 1170 & 47 & 1000 & 42 & 365 & 11 & 115 & 3 & 50 & 1 & $\mathrm{~g}$ \\
\hline $0010+405$ & 001331.0 & 405137 & 6890 & 347 & 4030 & 161 & 3260 & 102 & 1653 & 50 & 860 & 17 & 818 & 16 & $\mathrm{~g}$ \\
\hline $0013+387$ & 001600.0 & 390027 & 1690 & 93 & 940 & 38 & 740 & 37 & 268 & 8 & 82 & 2 & 42 & 1 & $\mathrm{Q}$ \\
\hline $0013+393$ & $00 \quad 1548.4$ & $\begin{array}{llll}39 & 37 & 07\end{array}$ & 720 & 54 & 410 & 17 & 270 & 31 & 106 & 3 & 31 & 1 & 16 & 1 & \\
\hline $0014+395$ & 001653.9 & $\begin{array}{lll}39 & 48 & 03\end{array}$ & 500 & 47 & 260 & 11 & 210 & 31 & 92 & 4 & 23 & 1 & 9 & 1 & \\
\hline $0015+399$ & $00 \quad 1741.5$ & 401629 & 280 & 42 & 140 & 7 & 140 & 30 & 63 & 2 & 35 & 1 & 32 & 1 & \\
\hline $0017+395$ & 001948.2 & 395154 & 390 & 45 & 200 & 9 & 150 & 30 & 60 & 2 & 24 & 4 & 9 & 1 & \\
\hline $0017+432$ & 002008.3 & 433010 & 2260 & 120 & 1100 & 44 & 800 & 38 & 256 & 8 & 63 & 6 & 27 & 1 & \\
\hline $0018+393$ & 002122.7 & 393833 & 430 & 45 & 280 & 12 & 200 & 31 & 88 & 3 & 20 & 1 & 18 & 1 & \\
\hline $0019+391$ & 002159.7 & 392546 & 790 & 56 & 400 & 17 & 290 & 31 & 87 & 3 & 18 & 1 & 6 & 1 & \\
\hline $0019+431$ & 002148.0 & 432825 & 5370 & 271 & 2880 & 115 & 2220 & 73 & 675 & 20 & 186 & 4 & 75 & 2 & Q \\
\hline $0020+437$ & 002331.3 & 440303 & 3180 & 164 & 1700 & 68 & 1220 & 47 & 391 & 12 & 106 & 2 & 44 & 1 & $\mathrm{~F}$ \\
\hline $0021+383$ & 002413.2 & $\begin{array}{llll}38 & 35 & 10\end{array}$ & 1210 & 73 & 730 & 30 & 590 & 35 & 208 & 6 & 65 & 2 & 29 & 1 & \\
\hline $0021+395$ & 002418.0 & 394906 & 450 & 46 & 200 & 9 & 160 & 30 & 63 & 2 & 16 & 1 & 10 & 1 & \\
\hline $22+390$ & 002526.2 & $39 \quad 1936$ & 1400 & 81 & 1230 & 49 & 1100 & 45 & 727 & 22 & 588 & 12 & 493 & 10 & Q \\
\hline $0022+394$ & 002503.9 & 394555 & 280 & 42 & 150 & 8 & 150 & 30 & 49 & 2 & 18 & 1 & 13 & 1 & \\
\hline $0022+399$ & 002442.9 & 401612 & & & 120 & 7 & 200 & 31 & 119 & 4 & 52 & 6 & 22 & 1 & Q \\
\hline $0022+424$ & 002535.2 & 424351 & 960 & 62 & 970 & 39 & 800 & 38 & 381 & 11 & 136 & 12 & 60 & 2 & $\mathrm{~g}$ \\
\hline $0023+382$ & 002609.5 & 383131 & 1220 & 73 & 770 & 31 & 650 & 36 & 273 & 8 & 94 & 2 & 48 & 1 & $\mathrm{~g}$ \\
\hline $0025+394$ & 002833.8 & 394441 & 350 & 44 & 140 & 7 & 120 & 30 & 39 & 1 & 12 & 1 & 6 & 1 & $\mathrm{~g}$ \\
\hline $0026+397$ & 002931.3 & 400314 & 390 & 45 & 160 & 8 & 160 & 30 & 32 & 1 & 8 & 1 & 4 & 1 & $\mathrm{~g}$ \\
\hline $0027+380$ & 003022.5 & $\begin{array}{llll}38 & 17 & 22\end{array}$ & 1560 & 88 & 760 & 31 & 580 & 35 & 186 & 6 & 47 & 1 & 22 & 1 & Q \\
\hline $27+395$ & 002944.0 & $3948 \quad 38$ & 920 & 61 & 560 & 23 & 410 & 32 & 192 & 6 & 76 & 2 & 38 & 1 & $\mathrm{Q}$ \\
\hline $28+390$ & 003137.8 & $\begin{array}{llll}39 & 19 & 04\end{array}$ & 810 & 57 & 550 & 22 & 470 & 33 & 160 & 5 & 50 & 1 & 27 & 1 & \\
\hline $28+394$ & 003111.3 & 394157 & 960 & 62 & 600 & 24 & 450 & 33 & 172 & 5 & 59 & 2 & 23 & 1 & \\
\hline $0028+409$ & 003048.8 & $41 \quad 1054$ & 3290 & 169 & 1560 & 63 & 1260 & 48 & 270 & 8 & 53 & 2 & 17 & 1 & G \\
\hline $28+450$ & 003050.8 & 452148 & 4240 & 216 & 2150 & 86 & 1620 & 57 & 461 & 14 & 114 & 3 & 38 & 1 & $\mathrm{~g}$ \\
\hline $0029+394$ & 003235.3 & $3942 \quad 15$ & 2240 & 119 & 1320 & 57 & 970 & 42 & 388 & 12 & 114 & 3 & 44 & 1 & \\
\hline $0029+398$ & $00 \quad 32 \quad 16.2$ & $4007 \quad 11$ & 410 & 45 & 340 & 14 & 290 & 31 & 143 & 4 & 62 & 6 & 57 & 2 & \\
\hline $0030+390$ & 003254.8 & 392016 & 480 & 47 & 250 & 11 & 200 & 31 & 47 & 1 & 12 & 1 & 6 & 1 & \\
\hline $0031+391$ & 003414.7 & 392414 & 14340 & 718 & 8180 & 327 & 6410 & 195 & 1881 & 56 & 419 & 8 & 139 & 3 & G \\
\hline $0031+393$ & 003411.6 & $\begin{array}{llll}39 & 36 & 14\end{array}$ & & & 250 & 11 & 260 & 31 & 86 & 4 & 28 & 5 & 20 & 1 & \\
\hline $32+390$ & 003511.0 & 391906 & 780 & 56 & 420 & 17 & 350 & 32 & 95 & 3 & 20 & 1 & 9 & 1 & \\
\hline $32+394$ & 003525.7 & 394046 & 820 & 57 & 470 & 19 & 350 & 32 & 149 & 5 & 52 & 2 & 20 & 1 & \\
\hline $32+423$ & 003505.9 & $4238 \quad 18$ & 1910 & 104 & 1140 & 46 & 920 & 41 & 353 & 11 & 110 & 2 & 60 & 2 & Q \\
\hline $33+397$ & 003601.5 & 400148 & 790 & 56 & 380 & 16 & 290 & 31 & 88 & 3 & 18 & 1 & 6 & 1 & \\
\hline $0033+425$ & 003638.5 & $4252 \quad 24$ & 2810 & 146 & 1520 & 61 & 1140 & 45 & 319 & 10 & 71 & 2 & 21 & 1 & \\
\hline $0034+387$ & $0037 \quad 19.3$ & $3859 \quad 16$ & 1230 & 73 & 640 & 26 & 540 & 34 & 168 & 5 & 51 & 1 & 22 & 1 & \\
\hline $0034+393$ & 003736.6 & $3938 \quad 12$ & & & 210 & 10 & 200 & 31 & 280 & 8 & 97 & 2 & 57 & 2 & Q \\
\hline $0034+444$ & 003653.5 & 444321 & 5370 & 271 & 2800 & 112 & 2250 & 74 & 679 & 20 & 192 & 4 & 69 & 2 & G \\
\hline $0035+385 \mathrm{~A}$ & 003746.9 & 384754 & 5410 & 273 & 3160 & 126 & 2550 & 82 & 827 & 25 & 258 & 28 & 127 & 3 & $\mathrm{~F}$ \\
\hline $6+398$ & 003932.9 & 400835 & 300 & 43 & 150 & 8 & 130 & 30 & 54 & 2 & 15 & 1 & 10 & 1 & \\
\hline $7+394$ & 004019.4 & 394451 & & & 90 & 6 & 120 & 30 & 55 & 2 & 20 & 1 & 12 & 1 & \\
\hline $37+396$ & $0040 \quad 17.2$ & 395505 & 350 & 44 & 170 & 8 & 140 & 30 & 66 & 2 & 18 & 1 & $<5$ & & \\
\hline $38+399$ & 004124.7 & 401231 & 540 & 48 & 220 & 10 & 220 & 31 & 86 & 3 & 28 & 4 & 15 & 1 & $\mathrm{~g}$ \\
\hline $0039+373$ & $\begin{array}{llll}00 & 42 & 07.1\end{array}$ & 373936 & 1650 & 92 & 2260 & 91 & 2000 & 67 & 943 & 28 & 258 & 5 & 89 & 2 & G \\
\hline $0039+391$ & 004155.0 & 392520 & 2210 & 118 & 1280 & 51 & 1020 & 43 & 317 & 10 & 76 & 2 & 24 & 1 & $\mathrm{G}$ \\
\hline $39+398$ & $00 \quad 42 \quad 17.4$ & $\begin{array}{lll}40 & 09 & 49\end{array}$ & 3000 & 155 & 2190 & 88 & 1980 & 67 & 753 & 23 & 221 & 5 & 86 & 2 & \\
\hline $39+412$ & $0042 \quad 18.7$ & 412927 & 1840 & 100 & 1190 & 48 & 910 & 41 & 377 & 11 & 117 & 3 & 61 & 2 & \\
\hline $10+470$ & 004324.2 & $47 \quad 1637$ & 4530 & 230 & 2410 & 97 & 1830 & 63 & 542 & 16 & 130 & 3 & 41 & 1 & G \\
\hline $1+382 \mathrm{~A}$ & 004349.4 & 383010 & 1440 & 82 & 830 & 34 & 660 & 36 & 224 & 7 & 56 & 2 & 18 & 1 & \\
\hline $1+393$ & 004358.4 & 393756 & 730 & 54 & 430 & 18 & 330 & 32 & 148 & 4 & 56 & 2 & 25 & 1 & \\
\hline $1+405$ & 004354.3 & 404635 & 790 & 56 & 520 & 21 & 420 & 33 & 168 & 5 & 67 & 2 & 37 & 1 & \\
\hline $41+425$ & 004439.2 & 424801 & 2550 & 134 & 1580 & 63 & 1260 & 48 & 467 & 14 & 125 & 3 & 56 & 1 & \\
\hline $0042+381 \mathrm{~A}$ & 004508.5 & $\begin{array}{lll}38 & 23 & 17\end{array}$ & & & 670 & 27 & 650 & 36 & 306 & 9 & 192 & 4 & 71 & 2 & \\
\hline $0042+381 \mathrm{~B}$ & $0045 \quad 17.5$ & $\begin{array}{lll}38 & 25 & 16\end{array}$ & & & 850 & 34 & 580 & 35 & 234 & 7 & 45 & 1 & 40 & 1 & \\
\hline $0042+386$ & $0044 \quad 45.3$ & 385700 & 1150 & 70 & 690 & 28 & 570 & 35 & 183 & 6 & 44 & 1 & 17 & 1 & \\
\hline $0043+398$ & 004555.7 & 400642 & & & 80 & 6 & 100 & 30 & 29 & 1 & 10 & 1 & 5 & 1 & $\mathrm{~g}$ \\
\hline $0045+393$ & $0048 \quad 02.0$ & 393726 & & & 200 & 9 & 220 & 31 & 215 & 6 & 104 & 2 & 77 & 2 & \\
\hline $45+395$ & 004755.3 & 394858 & 380 & 44 & 170 & 8 & 180 & 30 & 91 & 3 & 54 & 6 & 49 & 1 & BL \\
\hline $5+396$ & $0048 \quad 45.2$ & 395341 & 700 & 53 & 410 & 17 & 310 & 31 & 136 & 4 & 46 & 1 & 22 & 1 & g \\
\hline $45+400$ & $0048 \quad 12.8$ & 402153 & 3670 & 188 & 2120 & 85 & 1730 & 60 & 642 & 19 & 158 & 3 & 67 & 2 & $\mathrm{~g}$ \\
\hline $45+404$ & $0048 \quad 44.1$ & 404504 & 1320 & 77 & 670 & 27 & 560 & 34 & 223 & 7 & 76 & 2 & 28 & 1 & \\
\hline $46+439$ & 004850.7 & 441343 & 2550 & 134 & 1340 & 54 & 960 & 42 & 276 & 8 & 57 & 2 & 24 & 1 & $\mathrm{G}$ \\
\hline $0049+379$ & 005216.8 & $\begin{array}{llll}38 & 15 & 28\end{array}$ & 4520 & 230 & 3010 & 120 & 2550 & 82 & 797 & 24 & 158 & 3 & 56 & 2 & \\
\hline $0049+395$ & 005213.6 & 395124 & & & 40 & 5 & & & 26 & 4 & 5 & 1 & 5 & 1 & \\
\hline $0050+401$ & 005331.9 & 403106 & 4000 & 204 & 1450 & 58 & 1220 & 47 & 475 & 15 & 164 & 19 & 117 & 3 & $\mathrm{~g}$ \\
\hline
\end{tabular}


Table 2. continued

\begin{tabular}{|c|c|c|c|c|c|c|c|c|c|c|c|c|c|c|c|}
\hline B3 name & \begin{tabular}{|l}
$\mathrm{RA}(\mathrm{J} 2000)$ \\
{$\left[\begin{array}{ccc}\mathrm{h} & \mathrm{m} & \mathrm{s}\end{array}\right]$} \\
\end{tabular} & 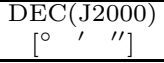 & $\begin{array}{r}S_{151} \\
{[\mathrm{mJy}]}\end{array}$ & $\begin{array}{r}\Delta S_{151} \\
{[\mathrm{mJy}]} \\
\end{array}$ & $\begin{array}{r}S_{327} \\
{[\mathrm{mJy}]}\end{array}$ & $\begin{array}{r}\Delta S_{327} \\
{[\mathrm{mJy}]} \\
\end{array}$ & $\begin{array}{r}S_{408} \\
{[\mathrm{mJy}]}\end{array}$ & $\begin{array}{r}\Delta S_{408} \\
{[\mathrm{mJy}]} \\
\end{array}$ & $\begin{array}{l}S_{1400} \\
{[\mathrm{mJy}]}\end{array}$ & $\begin{array}{r}S_{1400} \\
{[\mathrm{mJy}]} \\
\end{array}$ & $\begin{array}{l}S_{4850} \\
{[\mathrm{mJy}]}\end{array}$ & $\begin{array}{r}\Delta S_{4850} \\
{[\mathrm{mJy}]} \\
\end{array}$ & $\begin{array}{r}S_{10550} \\
{[\mathrm{mJy}]} \\
\end{array}$ & $\begin{array}{r}\Delta S_{10550} \\
{[\mathrm{mJy}]} \\
\end{array}$ & Id. \\
\hline $0051+397$ & \begin{tabular}{|llll}
00 & 54 & 00.1
\end{tabular} & 395860 & & & 160 & 8 & 150 & 30 & 58 & 2 & 18 & 1 & 8 & 1 & \\
\hline $0051+404$ & 005427.4 & 404211 & 5220 & 264 & 3100 & 124 & 2440 & 79 & 871 & 26 & 243 & 5 & 111 & 2 & \\
\hline $0052+380$ & 005456.0 & 382150 & 2760 & 144 & 1590 & 64 & 1250 & 48 & 396 & 12 & 100 & 2 & 32 & 1 & \\
\hline $0052+392$ & 005540.5 & 393336 & 1060 & 66 & 560 & 23 & 410 & 32 & 145 & 4 & 38 & 1 & 16 & 1 & \\
\hline $0052+395$ & 005540.6 & $\begin{array}{llll}39 & 48 & 16\end{array}$ & & & 430 & 18 & 280 & 31 & 146 & 4 & 41 & 1 & 24 & 1 & \\
\hline $0053+394$ & 005549.3 & 394501 & & & 780 & 32 & 600 & 35 & 255 & 8 & 70 & 2 & 26 & 1 & $\mathrm{~g}$ \\
\hline $0053+439$ & 005646.4 & 441324 & 3840 & 196 & 1940 & 78 & 1350 & 50 & 436 & 13 & 116 & 3 & 55 & 1 & \\
\hline $0054+396$ & 005729.4 & $\begin{array}{llll}39 & 57 & 17\end{array}$ & 590 & 50 & 360 & 15 & 280 & 31 & 98 & 3 & 26 & 1 & 14 & 1 & \\
\hline $0056+389$ & 005938.6 & 391022 & 610 & 50 & 330 & 14 & 220 & 31 & 97 & 4 & 24 & 1 & 8 & 1 & \\
\hline $0057+395$ & $\begin{array}{lll}01 & 00 & 05.3\end{array}$ & 394935 & 380 & 44 & 220 & 10 & 190 & 31 & 76 & 2 & 27 & 1 & 15 & 1 & \\
\hline $0057+397$ & 005951.0 & 395847 & 390 & 45 & 150 & 8 & 110 & 30 & 10 & 2 & $<3$ & 1 & $<3$ & & \\
\hline $0058+403$ & $\begin{array}{llll}01 & 00 & 53.2\end{array}$ & 403600 & 1530 & 86 & 1070 & 43 & 760 & 38 & 267 & 8 & 80 & 2 & 33 & 1 & \\
\hline $0059+397$ & $\begin{array}{lll}01 & 02 & 26.8\end{array}$ & 400421 & & & 160 & 8 & 110 & 30 & 45 & 1 & 15 & 1 & 8 & 1 & \\
\hline $0059+461$ & $\begin{array}{lll}01 & 02 & 46.6\end{array}$ & 462437 & 8600 & 432 & 4960 & 198 & 3920 & 121 & 1293 & 39 & 289 & 6 & 112 & 2 & \\
\hline $0100+388$ & $\begin{array}{llll}0 & 03 & 43.4\end{array}$ & 390440 & 1410 & 81 & 790 & 32 & 570 & 35 & 194 & 6 & 56 & 2 & 20 & 1 & \\
\hline $0100+397$ & $\begin{array}{llll}0 & 01 & 02 & 58.1\end{array}$ & 400002 & & & 140 & 7 & 110 & 30 & 43 & 1 & 8 & 1 & 6 & 1 & \\
\hline $0103+422$ & $\begin{array}{llll}01 & 06 & 42.4\end{array}$ & $42 \quad 2955$ & 5300 & 268 & 3100 & 124 & 2370 & 77 & 817 & 25 & 207 & 4 & 76 & 2 & \\
\hline $0105+441$ & $\begin{array}{llll}01 & 08 & 10.3\end{array}$ & 442501 & 2430 & 128 & 1480 & 59 & 1150 & 46 & 402 & 12 & 138 & 3 & 78 & 2 & \\
\hline $0106+380$ & $\begin{array}{lll}01 & 09 & 25.6\end{array}$ & $\begin{array}{lll}38 & 16 & 44\end{array}$ & 800 & 57 & 560 & 23 & 450 & 33 & 189 & 6 & 84 & 2 & 40 & 1 & Q \\
\hline $0106+397$ & $\mid \begin{array}{lll}01 & 09 & 25.2\end{array}$ & 400002 & 960 & 62 & 610 & 25 & 460 & 33 & 163 & 5 & 43 & 1 & 14 & 1 & \\
\hline $0107+397$ & $\begin{array}{llll}01 & 09 & 51.0\end{array}$ & 395828 & 1370 & 79 & 760 & 31 & 610 & 35 & 199 & 6 & 60 & 2 & 25 & 1 & \\
\hline $0107+398$ & $\begin{array}{llll}01 & 10 & 44.4\end{array}$ & $40 \quad 0722$ & & & 120 & 7 & 110 & 30 & 39 & 1 & 15 & 1 & 13 & 1 & Q \\
\hline $0107+399$ & $\begin{array}{lll}01 & 10 & 03.5\end{array}$ & $4011 \quad 13$ & 510 & 47 & 310 & 13 & 260 & 31 & 73 & 2 & 11 & 1 & 6 & 1 & \\
\hline $0108+396$ & $\begin{array}{llll}01 & 11 & 01.8\end{array}$ & 395206 & 860 & 59 & 440 & 18 & 320 & 31 & 113 & 3 & 30 & 4 & 11 & 1 & \\
\hline $0108+402$ & 011104.9 & 403343 & 1430 & 82 & 770 & 31 & 660 & 36 & 207 & 6 & 60 & 2 & 22 & 1 & \\
\hline $0109+390$ & $01 \quad 1235.9$ & $\begin{array}{llll}39 & 18 & 04\end{array}$ & 670 & 52 & 350 & 15 & 280 & 31 & 81 & 2 & 22 & 4 & 7 & 1 & \\
\hline $0109+415$ & $\begin{array}{llll}01 & 12 & 08.3\end{array}$ & 414660 & 2350 & 124 & 1390 & 56 & 1140 & 45 & 541 & 16 & 197 & 4 & 107 & 2 & $\mathrm{~g}$ \\
\hline $0109+416 \mathrm{~B}$ & $\begin{array}{llll}01 & 12 & 15.4\end{array}$ & 415513 & 4170 & 212 & 2620 & 105 & 1960 & 66 & 856 & 26 & 247 & 27 & 60 & 2 & $\mathrm{~g}$ \\
\hline $0110+386$ & $01 \quad 1257.4$ & $\begin{array}{lll}38 & 5317\end{array}$ & 1200 & 72 & 580 & 24 & 490 & 33 & 174 & 5 & 56 & 2 & 22 & 1 & \\
\hline $0110+395$ & 011337.6 & 395033 & 860 & 59 & 390 & 16 & 300 & 31 & 109 & 3 & 48 & 5 & 39 & 1 & \\
\hline $0110+398$ & $\begin{array}{lll}01 & 13 & 28.7\end{array}$ & 401028 & 300 & 43 & 190 & 9 & 130 & 30 & 46 & 1 & 14 & 1 & 6 & 1 & \\
\hline $0110+401$ & $\begin{array}{llll}01 & 13 & 17.7\end{array}$ & $40 \quad 2612$ & 1820 & 99 & 1290 & 52 & 1080 & 44 & 551 & 17 & 233 & 5 & 144 & 3 & Q \\
\hline $0112+400$ & $\begin{array}{llll}01 & 15 & 15.1\end{array}$ & 402011 & 1220 & 73 & 660 & 27 & 540 & 34 & 164 & 5 & 46 & 1 & 18 & 1 & \\
\hline $0112+432$ & $01 \quad 1457.2$ & 433216 & 2490 & 131 & 1400 & 56 & 1090 & 44 & 376 & 11 & 110 & 2 & 42 & 1 & \\
\hline $0113+400$ & 011624.6 & 401725 & 3870 & 198 & 2210 & 89 & 1850 & 63 & 684 & 21 & 199 & 4 & 93 & 2 & \\
\hline $0114+399$ & \begin{tabular}{l|lll}
01 & 17 & 27.8
\end{tabular} & 401250 & 990 & 64 & 470 & 19 & 440 & 33 & 126 & 4 & 38 & 1 & 15 & 1 & \\
\hline $0115+394$ & 011755.4 & 394433 & 960 & 62 & 420 & 17 & 290 & 31 & 78 & 2 & 18 & 1 & 7 & 1 & \\
\hline $0115+453 \mathrm{~A}$ & 011759.5 & 453622 & 11400 & 571 & 5740 & 230 & 4270 & 132 & 1268 & 38 & 346 & 7 & 144 & 3 & G \\
\hline $0115+469$ & $\begin{array}{llll}01 & 18 & 22.7\end{array}$ & $47 \quad 12 \quad 21$ & 4320 & 220 & 2240 & 90 & 1770 & 61 & 573 & 17 & 151 & 18 & 64 & 2 & \\
\hline $0116+397$ & $\begin{array}{llll}01 & 19 & 17.5\end{array}$ & 395851 & 480 & 47 & 230 & 10 & 210 & 31 & 68 & 2 & 17 & 1 & 7 & 1 & \\
\hline $0116+438$ & \begin{tabular}{l|lll}
01 & 19 & 22.8
\end{tabular} & 440739 & 2380 & 126 & 1420 & 57 & 1050 & 44 & 426 & 13 & 150 & 3 & 71 & 2 & $\mathrm{~g}$ \\
\hline $0119+395$ & 012227.5 & 394826 & 540 & 48 & 300 & 13 & 210 & 31 & 64 & 3 & 18 & 1 & 8 & 1 & \\
\hline $0119+397$ & 012234.0 & 400106 & 960 & 62 & 540 & 22 & 480 & 33 & 178 & 5 & 65 & 2 & 30 & 1 & \\
\hline $0120+380$ & 012252.0 & $\begin{array}{llll}38 & 18 & 20\end{array}$ & 1720 & 95 & 710 & 29 & 540 & 34 & 140 & 4 & 33 & 1 & 10 & 1 & \\
\hline $0120+405$ & 012326.0 & 404659 & 3520 & 180 & 2020 & 81 & 1680 & 59 & 614 & 18 & 157 & 3 & 65 & 2 & $\mathrm{G}$ \\
\hline $0121+389$ & 012408.8 & 391326 & 950 & 62 & 560 & 23 & 450 & 33 & 170 & 5 & 52 & 2 & 20 & 1 & \\
\hline $0122+395$ & 012528.3 & 394552 & 390 & 45 & 180 & 9 & 160 & 30 & 71 & 2 & 21 & 4 & 9 & 1 & \\
\hline $0123+385$ & $\begin{array}{llll}01 & 26 & 10.8\end{array}$ & 385046 & 1100 & 68 & 560 & 23 & 490 & 33 & 155 & 5 & 46 & 1 & 16 & 1 & G \\
\hline $0123+396$ & 012625.8 & 395413 & 590 & 50 & 380 & 16 & 330 & 32 & 174 & 5 & 77 & 2 & 40 & 1 & $\mathrm{~g}$ \\
\hline $0123+402$ & 012559.1 & 402836 & 1830 & 100 & 970 & 39 & 810 & 39 & 258 & 8 & 67 & 2 & 25 & 1 & \\
\hline $0124+387$ & $\begin{array}{llll}01 & 27 & 44.4\end{array}$ & 390011 & 840 & 58 & 530 & 22 & 430 & 33 & 157 & 5 & 48 & 1 & 16 & 1 & \\
\hline $0126+392 \mathrm{~A}$ & 012928.1 & 392956 & 920 & 61 & 400 & 17 & 320 & 31 & 92 & 3 & 17 & 1 & 7 & 1 & \\
\hline $0127+395$ & 013042.4 & 395015 & & & 130 & 7 & 100 & 30 & 48 & 2 & 14 & 1 & 6 & 1 & \\
\hline $0127+399$ & $\begin{array}{llll}01 & 30 & 49.4\end{array}$ & 401008 & 1040 & 66 & 450 & 19 & 340 & 32 & 96 & 3 & 28 & 1 & 9 & 1 & \\
\hline $0128+394$ & 013129.6 & 394258 & 1940 & 105 & 1140 & 46 & 920 & 41 & 326 & 10 & 84 & 2 & 25 & 1 & \\
\hline $0130+381$ & 013343.0 & 382260 & 2000 & 108 & 1260 & 51 & 1070 & 44 & 430 & 13 & 173 & 4 & 105 & 2 & $\mathrm{~g}$ \\
\hline $0130+384$ & 013259.8 & 384102 & 1270 & 75 & 660 & 27 & 530 & 34 & 185 & 6 & 66 & 2 & 33 & 1 & Q \\
\hline $0130+398$ & 013312.9 & 400322 & 520 & 48 & 310 & 13 & 280 & 31 & 119 & 4 & 47 & 5 & 25 & 1 & \\
\hline $0131+390$ & 013445.1 & 391953 & 1070 & 67 & 590 & 24 & 470 & 33 & 187 & 6 & 48 & 1 & 27 & 1 & $\mathrm{~g}$ \\
\hline $0132+376 \mathrm{~A}$ & 013529.5 & $3754 \quad 13$ & 10300 & 517 & 5390 & 216 & 4320 & 133 & 1335 & 40 & 357 & 37 & 197 & 4 & $\mathrm{~g}$ \\
\hline $0132+392$ & 013534.5 & $\begin{array}{llll}39 & 28 & 13\end{array}$ & 840 & 58 & 490 & 20 & 350 & 32 & 138 & 4 & 47 & 1 & 21 & 1 & \\
\hline $0133+381$ & 013638.5 & 382602 & 1330 & 78 & 560 & 23 & 470 & 33 & 142 & 4 & 45 & 1 & 21 & 1 & \\
\hline $0134+386$ & 013749.6 & 385128 & 2300 & 122 & 1420 & 57 & 1170 & 46 & 460 & 14 & 170 & 4 & 78 & 2 & $\mathrm{~g}$ \\
\hline $0134+389$ & $\begin{array}{llll}01 & 37 & 15.9\end{array}$ & 391136 & 610 & 50 & 360 & 15 & 310 & 31 & 112 & 3 & 50 & 6 & 17 & 1 & \\
\hline $0136+396$ & 013930.9 & 395708 & 10800 & 541 & 4960 & 198 & 3890 & 120 & 797 & 24 & 277 & 13 & 115 & 3 & $\mathrm{~g}$ \\
\hline $0137+385$ & $\begin{array}{lll}01 & 40 & 46.7\end{array}$ & 384848 & 1000 & 64 & 510 & 21 & 420 & 33 & 125 & 4 & 37 & 1 & 20 & 1 & \\
\hline $0137+401$ & \begin{tabular}{l|lll}
01 & 40 & 33.8
\end{tabular} & 402414 & 1790 & 98 & 970 & 39 & 820 & 39 & 289 & 9 & 99 & 2 & 50 & 1 & Q \\
\hline $0138+394$ & 014128.6 & 394038 & 990 & 64 & 500 & 21 & 430 & 33 & 118 & 4 & 50 & 1 & 14 & 1 & \\
\hline $0139+389 \mathrm{~A}$ & \begin{tabular}{|lll}
01 & 42 & 09.8
\end{tabular} & $\begin{array}{lll}39 & 12 \quad 40\end{array}$ & 710 & 53 & 510 & 21 & 430 & 33 & 216 & 7 & 85 & 2 & 42 & 1 & Q \\
\hline $0140+387$ & 014333.0 & $\begin{array}{lll}39 & 02 & 10\end{array}$ & 4860 & 246 & 2480 & 99 & 1810 & 62 & 430 & 13 & 69 & 2 & 18 & 1 & \\
\hline $0141+398$ & 014431.9 & 400313 & 770 & 56 & 360 & 15 & 290 & 31 & 91 & 3 & 24 & 1 & 11 & 1 & \\
\hline $0143+393$ & $\begin{array}{llll}01 & 46 & 00.4\end{array}$ & 393315 & 950 & 62 & 440 & 18 & 380 & 32 & 92 & 3 & 22 & 1 & 7 & 1 & \\
\hline $0143+446 \mathrm{~B}$ & $\begin{array}{llll}01 & 46 & 48.6\end{array}$ & 445523 & 4120 & 210 & 2240 & 90 & 1710 & 59 & 587 & 18 & 206 & 23 & 36 & 1 & Q \\
\hline $0144+391$ & 014722.6 & 392449 & 380 & 44 & 310 & 13 & 250 & 31 & 97 & 3 & 33 & 5 & 13 & 1 & \\
\hline $0144+399$ & $\begin{array}{llll}01 & 47 & 16.7\end{array}$ & $40 \quad 1349$ & 590 & 50 & 270 & 12 & 210 & 31 & 88 & 3 & 22 & 4 & 9 & 1 & \\
\hline $0144+430$ & 014757.7 & 431943 & 2210 & 118 & 1170 & 47 & 920 & 41 & 287 & 9 & 83 & 2 & 32 & 1 & $\mathrm{Q}$ \\
\hline $0144+432$ & 014755.7 & 433215 & 1580 & 89 & 1020 & 41 & 820 & 39 & 336 & 10 & 115 & 3 & 51 & 1 & Q \\
\hline $0146+394$ & $\begin{array}{lll}01 & 49 & 45.9\end{array}$ & 393912 & & & 380 & 16 & 240 & 31 & 112 & 5 & 28 & 5 & 15 & 1 & \\
\hline $0147+397$ & $\begin{array}{llll}01 & 50 & 15.4 \\
\end{array}$ & 395937 & 520 & 48 & 280 & 12 & 260 & 31 & 101 & 3 & 39 & 5 & 22 & 1 & $g$ \\
\hline
\end{tabular}


Table 2. continued

\begin{tabular}{|c|c|c|c|c|c|c|c|c|c|c|c|c|c|c|c|}
\hline B3 name & \begin{tabular}{|ccc}
$\mathrm{RA}(\mathrm{J} 2000)$ \\
{$\left[\begin{array}{lll}\mathrm{h} & \mathrm{m} & \mathrm{s}\end{array}\right]$} \\
\end{tabular} & 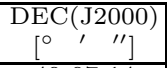 & $\begin{array}{r}S_{151} \\
{[\mathrm{mJy}]}\end{array}$ & $\begin{array}{r}\Delta S_{151} \\
{[\mathrm{mJy}]} \\
\end{array}$ & $\begin{array}{r}S_{327} \\
{[\mathrm{mJy}]}\end{array}$ & $\begin{array}{r}\Delta S_{327} \\
{[\mathrm{mJy}]}\end{array}$ & $\begin{array}{r}S_{408} \\
{[\mathrm{mJy}]}\end{array}$ & $\begin{array}{r}\Delta S_{408} \\
{[\mathrm{mJy}]}\end{array}$ & $\begin{array}{l}S_{1400} \\
{[\mathrm{mJy}]}\end{array}$ & $\begin{array}{r}\Delta S_{1400} \\
{[\mathrm{mJy}]} \\
\end{array}$ & $\begin{array}{l}S_{4850} \\
{[\mathrm{mJy}]}\end{array}$ & $\begin{array}{r}\Delta S_{4850} \\
{[\mathrm{mJy}]} \\
\end{array}$ & $\begin{array}{l}S_{10550} \\
{[\mathrm{mJy}]} \\
\end{array}$ & $\begin{array}{r}\Delta S_{10550} \\
{[\mathrm{mJy}]} \\
\end{array}$ & Id. \\
\hline $0147+398$ & 015036.3 & 400714 & & & 100 & 6 & 120 & 30 & 46 & 1 & 18 & 1 & 11 & 1 & $\overline{\mathrm{g}}$ \\
\hline $0147+400$ & 015019.6 & 401729 & 1880 & 102 & 1560 & 63 & 1540 & 55 & 723 & 22 & 277 & 6 & 163 & 3 & \\
\hline $0149+398$ & 015208.4 & 400434 & 970 & 63 & 650 & 26 & 590 & 35 & 270 & 8 & 117 & 3 & 79 & 2 & $\mathrm{~g}$ \\
\hline $0150+406$ & 015349.1 & 405553 & 2770 & 144 & 960 & 39 & 830 & 39 & 247 & 7 & 59 & 2 & 27 & 1 & \\
\hline $0152+382$ & $\begin{array}{llll}01 & 55 & 17.8\end{array}$ & 383119 & 1450 & 83 & 810 & 33 & 640 & 36 & 200 & 6 & 78 & 2 & 59 & 2 & \\
\hline $0152+435$ & 015530.5 & 434601 & 11800 & 591 & 6570 & 263 & 5270 & 161 & 1813 & 54 & 495 & 10 & 252 & 5 & G \\
\hline $0153+417$ & 015621.2 & $4202 \quad 28$ & 4790 & 243 & 2670 & 107 & 2140 & 71 & 753 & 23 & 203 & 4 & 100 & 2 & $\mathrm{~g}$ \\
\hline $0157+393 \mathrm{~A}$ & $\begin{array}{llll}02 & 00 & 09.6\end{array}$ & 393355 & 830 & 58 & 520 & 21 & 320 & 31 & 111 & 3 & 29 & 1 & 13 & 1 & $\mathrm{G}$ \\
\hline $0157+393 \mathrm{~B}$ & $\mid \begin{array}{lll}0 & 00 & 51.1\end{array}$ & 393448 & 2300 & 122 & 1820 & 83 & 1120 & 45 & 615 & 18 & 232 & 46 & 164 & 3 & $\mathrm{~g}$ \\
\hline $0157+405 \mathrm{~A}$ & $\mid \begin{array}{llll}02 & 00 & 30.1\end{array}$ & 404853 & 7070 & 356 & 4360 & 184 & 4390 & 135 & 220 & 10 & 418 & 10 & 95 & 2 & $\mathrm{~g}$ \\
\hline $0157+442$ & $\begin{array}{llll}02 & 00 & 38.9\end{array}$ & $4427 \quad 16$ & 6340 & 320 & 3900 & 156 & 3260 & 102 & 1273 & 38 & 348 & 7 & 147 & 3 & Q \\
\hline $0158+391$ & $\mid \begin{array}{lll}02 & 01 & 51.2\end{array}$ & 392424 & 450 & 46 & 280 & 12 & 260 & 31 & 94 & 3 & 35 & 1 & 18 & 1 & \\
\hline $0158+394$ & $\begin{array}{lll}02 & 02 & 01.7\end{array}$ & 394321 & & & 110 & 6 & 120 & 30 & 103 & 3 & 139 & 3 & 144 & 3 & Q \\
\hline $0159+390$ & $\begin{array}{lll}02 & 02 & 52.8\end{array}$ & 391640 & 470 & 46 & 260 & 11 & 240 & 31 & 98 & 4 & 33 & 1 & 20 & 1 & $\mathrm{~g}$ \\
\hline $0159+397$ & $\begin{array}{lll}02 & 02 & 20.0\end{array}$ & 395938 & 450 & 46 & 290 & 12 & 230 & 31 & 92 & 3 & 26 & 1 & 10 & 1 & \\
\hline $0200+393$ & 020350.3 & 393242 & 1170 & 71 & 740 & 30 & 590 & 35 & 283 & 9 & 104 & 2 & 50 & 1 & \\
\hline $0201+390$ & 020444.7 & 391925 & 550 & 49 & 340 & 14 & 270 & 31 & 122 & 4 & 39 & 5 & 15 & 1 & \\
\hline $0201+396$ & $\mid \begin{array}{lll}0 & 04 & 38.2\end{array}$ & 395519 & 780 & 56 & 330 & 14 & 280 & 31 & 60 & 2 & 13 & 1 & 4 & 1 & \\
\hline $0201+402$ & $\mid \begin{array}{lll}02 & 04 & 39.1\end{array}$ & $40 \quad 2942$ & 880 & 59 & 550 & 22 & 470 & 33 & 170 & 5 & 55 & 2 & 23 & 1 & \\
\hline $0202+380$ & $\mid \begin{array}{lll}02 & 05 & 53.2\end{array}$ & $38 \quad 1543$ & 1170 & 71 & 630 & 26 & 440 & 33 & 194 & 7 & 69 & 14 & 42 & 1 & $\mathrm{~g}$ \\
\hline $205+395$ & \begin{tabular}{|llll}
02 & 08 & 07.3
\end{tabular} & 394500 & 850 & 58 & 570 & 23 & 470 & 33 & 184 & 6 & 69 & 2 & 34 & 1 & \\
\hline $205+398$ & $\mid \begin{array}{lll}02 & 08 & 56.2\end{array}$ & 400701 & 310 & 43 & 110 & 6 & 100 & 30 & 31 & 1 & 11 & 1 & 3 & 1 & \\
\hline $207+389$ & $\mid \begin{array}{lll}02 & 10 & 09.5\end{array}$ & 391123 & 430 & 45 & 310 & 13 & 220 & 31 & 107 & 3 & 41 & 5 & 19 & 1 & $\mathrm{~g}$ \\
\hline $207+395$ & $\begin{array}{llll}02 & 10 & 10.6\end{array}$ & 395000 & 630 & 51 & 420 & 17 & 360 & 32 & 138 & 4 & 48 & 1 & 19 & 1 & $\mathrm{Q}$ \\
\hline $0207+397$ & $\mid \begin{array}{lll}02 & 10 & 56.1\end{array}$ & 400139 & 730 & 54 & 450 & 19 & 390 & 32 & 140 & 4 & 57 & 2 & 30 & 1 & $\mathrm{~g}$ \\
\hline $0207+399$ & $\mid \begin{array}{llll}02 & 10 & 16.3\end{array}$ & 401334 & & & 110 & 6 & 100 & 30 & 49 & 2 & 20 & 1 & 13 & 1 & \\
\hline $0209+386$ & \begin{tabular}{|lll}
02 & 12 & 43.8
\end{tabular} & 385052 & 1310 & 77 & 820 & 33 & 640 & 36 & 219 & 7 & 65 & 2 & 23 & 1 & \\
\hline $0209+390$ & $\begin{array}{lll}02 & 12 & 50.5\end{array}$ & $\begin{array}{lll}39 & 17 & 19\end{array}$ & 540 & 48 & 290 & 12 & 230 & 31 & 64 & 2 & 14 & 1 & 6 & 1 & \\
\hline $209+394$ & $\begin{array}{lll}02 & 12 & 37.5\end{array}$ & $3942 \quad 18$ & & & 160 & 8 & 130 & 30 & 76 & 2 & 76 & 7 & 42 & 1 & $\mathrm{Q}$ \\
\hline $210+396$ & $\mid \begin{array}{lll}02 & 13 & 34.2\end{array}$ & 395407 & 330 & 43 & 200 & 9 & 160 & 30 & 50 & 2 & 13 & 1 & 8 & 1 & \\
\hline $211+393$ & $\mid \begin{array}{lll}02 & 14 & 06.2\end{array}$ & 393300 & 1700 & 94 & 880 & 35 & 520 & 34 & 238 & 8 & 73 & 2 & 28 & 1 & $\mathrm{~g}$ \\
\hline $213+392$ & $\mid \begin{array}{lll}02 & 16 & 13.2\end{array}$ & 393054 & 880 & 59 & 400 & 17 & 310 & 31 & 105 & 3 & 30 & 1 & 14 & 1 & $\mathrm{G}$ \\
\hline $213+398$ & $\mid \begin{array}{lll}02 & 16 & 23.0\end{array}$ & 400533 & 470 & 46 & 240 & 11 & 210 & 31 & 66 & 2 & 18 & 1 & 10 & 1 & G \\
\hline $0213+407$ & $\mid \begin{array}{lll}02 & 16 & 52.1\end{array}$ & 410054 & 990 & 64 & 650 & 26 & 510 & 34 & 180 & 5 & 41 & 1 & 18 & 1 & $\mathrm{~g}$ \\
\hline $0213+412$ & $\mid \begin{array}{lll}0 & 16 & 30.3\end{array}$ & 413151 & 2160 & 115 & 1510 & 61 & 1260 & 48 & 544 & 16 & 196 & 4 & 116 & 3 & G \\
\hline $0214+393$ & $\begin{array}{llll}02 & 17 & 38.3\end{array}$ & 393618 & 1420 & 81 & 1150 & 50 & 770 & 38 & 367 & 11 & 132 & 3 & 86 & 2 & $\mathrm{~g}$ \\
\hline $0216+388$ & $\begin{array}{lll}02 & 19 & 53.0\end{array}$ & 390545 & 650 & 52 & 390 & 16 & 300 & 31 & 74 & 2 & 18 & 1 & 7 & 1 & \\
\hline $0216+393$ & $\begin{array}{lll}02 & 19 & 35.3\end{array}$ & 393237 & & & 160 & 8 & 200 & 31 & 130 & 4 & 65 & 2 & 34 & 1 & \\
\hline $216+403$ & $\begin{array}{lll}02 & 20 & 03.5\end{array}$ & 403543 & 1070 & 67 & 700 & 35 & 570 & 35 & 249 & 7 & 95 & 2 & 36 & 1 & \\
\hline $0216+423$ & $\mid \begin{array}{lll}02 & 19 & 09.3\end{array}$ & 423260 & 4950 & 251 & 3100 & 124 & 2300 & 75 & 786 & 24 & 221 & 5 & 117 & 3 & \\
\hline $0217+395$ & $\begin{array}{lll}02 & 20 & 08.3\end{array}$ & 394440 & & & 230 & 10 & 160 & 30 & 63 & 2 & 20 & 1 & 10 & 1 & \\
\hline $0217+417$ & $\mid \begin{array}{lll}02 & 20 & 14.4\end{array}$ & 415735 & 1880 & 102 & 1060 & 43 & 850 & 39 & 311 & 9 & 96 & 2 & 55 & 1 & Q \\
\hline $0218+396$ & $02 \quad 2148.4$ & 395533 & 6040 & 305 & 3420 & 137 & 2930 & 93 & 966 & 29 & 249 & 27 & 157 & 3 & \\
\hline $0218+399 \mathrm{~A}$ & 022146.7 & 400904 & 2290 & 121 & 1050 & 58 & 850 & 39 & 234 & 7 & 58 & 2 & 20 & 1 & \\
\hline $218+402 \mathrm{~A}$ & $\begin{array}{lll}02 & 22 & 01.9\end{array}$ & 402816 & 1130 & 69 & 730 & 30 & 690 & 36 & 233 & 7 & 77 & 2 & 22 & 1 & \\
\hline $19+397$ & $\begin{array}{lll}02 & 22 & 39.3\end{array}$ & 400040 & 830 & 58 & 500 & 21 & 440 & 33 & 118 & 4 & 44 & 1 & 19 & 1 & \\
\hline $0219+421$ & $\mid \begin{array}{lll}02 & 22 & 32.9\end{array}$ & $42 \quad 2055$ & 3300 & 170 & 2430 & 105 & 1180 & 46 & 286 & 9 & 350 & 36 & 152 & 3 & $\mathrm{~g}$ \\
\hline $219+428 \mathrm{~A}$ & $\mid \begin{array}{lll}02 & 22 & 39.7\end{array}$ & $4302 \quad 10$ & 10670 & 535 & 6580 & 264 & 5050 & 154 & 2378 & 71 & 1336 & 27 & 1235 & 25 & BL \\
\hline $219+443$ & $\begin{array}{lll}02 & 22 & 17.6\end{array}$ & 443257 & 2210 & 118 & 1130 & 45 & 890 & 40 & 283 & 9 & 93 & 2 & 64 & 2 & Q \\
\hline $0220+388$ & $\mid \begin{array}{lll}02 & 23 & 09.4\end{array}$ & $\begin{array}{lll}39 & 0308\end{array}$ & 980 & 63 & 670 & 27 & 540 & 34 & 159 & 5 & 41 & 1 & 13 & 1 & \\
\hline $0220+393 \mathrm{~A}$ & $02 \quad 2333.9$ & 393560 & 2490 & 131 & 1580 & 63 & 1040 & 43 & 529 & 16 & 241 & 5 & 170 & 4 & $\mathrm{~g}$ \\
\hline $0220+397$ & $\begin{array}{lll}02 & 23 & 42.8\end{array}$ & 400052 & 16940 & 848 & 10890 & 436 & 9080 & 274 & 3045 & 91 & 729 & 15 & 290 & 6 & $\mathrm{G}$ \\
\hline $0220+427 \mathrm{~A}$ & $02 \quad 23 \quad 16.5$ & 425939 & 20950 & 1048 & 21410 & 856 & 14830 & 446 & 6214 & 186 & 3060 & 306 & 1806 & 36 & $\mathrm{~g}$ \\
\hline $221+383$ & $02 \quad 2416.9$ & 383208 & 1080 & 67 & 570 & 23 & 430 & 33 & 153 & 5 & 55 & 11 & 29 & 1 & \\
\hline $221+393$ & $02 \quad 2443.2$ & 393247 & 640 & 51 & 270 & 12 & 210 & 31 & 53 & 4 & 7 & 2 & 8 & 1 & \\
\hline $221+396$ & $02 \quad 2450.7$ & 395516 & & & 180 & 9 & 220 & 31 & 58 & 2 & 32 & 4 & 33 & 1 & \\
\hline $222+403$ & $\begin{array}{lll}02 & 25 & 44.8\end{array}$ & 403135 & 4050 & 206 & 2490 & 100 & 2190 & 72 & 941 & 28 & 351 & 36 & 182 & 4 & $\mathrm{~g}$ \\
\hline $0222+422 \mathrm{~A}$ & $\mid \begin{array}{lll}02 & 25 & 32.2\end{array}$ & $42 \quad 2941$ & 2000 & 108 & 1130 & 45 & 810 & 39 & 262 & 8 & 66 & 2 & 23 & 1 & \\
\hline $0224+393$ & $\mid \begin{array}{lll}02 & 27 & 07.2\end{array}$ & 393142 & 1290 & 76 & 840 & 34 & 890 & 40 & 433 & 13 & 206 & 4 & 186 & 4 & Q \\
\hline $0224+396$ & $02 \quad 2758.8$ & 394952 & & & 200 & 9 & 180 & 30 & 70 & 3 & 31 & 1 & 12 & 1 & \\
\hline $0225+381$ & $\mid \begin{array}{lll}02 & 28 & 30.2\end{array}$ & 382113 & 960 & 62 & 590 & 24 & 470 & 33 & 219 & 7 & 67 & 2 & 30 & 1 & $\mathrm{~g}$ \\
\hline $0225+389$ & $\mid \begin{array}{lll}02 & 29 & 00.1\end{array}$ & $\begin{array}{lll}39 & 09 & 03\end{array}$ & 360 & 44 & 240 & 11 & 220 & 31 & 101 & 5 & 45 & 11 & 35 & 1 & $\mathrm{Q}$ \\
\hline $225+427$ & $02 \quad 2855.1$ & 430053 & 1490 & 85 & 1030 & 41 & 860 & 40 & 305 & 9 & 95 & 2 & 43 & 1 & G \\
\hline $226+394$ & $02 \quad 2947.7$ & 394248 & 880 & 59 & 470 & 19 & 400 & 32 & 153 & 5 & 62 & 2 & 31 & 1 & $\mathrm{~g}$ \\
\hline $226+396$ & $02 \quad 2956.9$ & 395306 & 630 & 51 & 280 & 12 & 210 & 31 & 41 & 1 & 6 & 1 & 2 & 1 & \\
\hline $226+467$ & $\begin{array}{lll}02 & 29 & 21.6\end{array}$ & $\begin{array}{lll}47 & 00 & 19\end{array}$ & 4990 & 253 & 3240 & 130 & 2450 & 79 & 893 & 27 & 279 & 6 & 108 & 2 & $\mathrm{Q}$ \\
\hline $0227+397$ & $\mid \begin{array}{lll}02 & 30 & 30.2\end{array}$ & 395845 & 460 & 46 & 300 & 13 & 230 & 31 & 87 & 3 & 34 & 5 & 8 & 1 & \\
\hline $0227+398$ & $\mid \begin{array}{lll}02 & 31 & 07.4\end{array}$ & 400331 & 1120 & 69 & 840 & 34 & 680 & 36 & 241 & 7 & 72 & 2 & 30 & 1 & \\
\hline $0228+392$ & $\begin{array}{llll}02 & 31 & 07.8\end{array}$ & 392721 & 1090 & 68 & 570 & 23 & 380 & 32 & 132 & 4 & 36 & 1 & 12 & 1 & \\
\hline $0228+393$ & $\mid \begin{array}{lll}02 & 31 & 49.1\end{array}$ & 393301 & 6270 & 316 & 3510 & 140 & 2620 & 84 & 777 & 23 & 212 & 4 & 86 & 2 & G \\
\hline $0228+409 \mathrm{~A}$ & 023138.8 & 410954 & 1740 & 96 & 1160 & 47 & 900 & 40 & 355 & 11 & 111 & 2 & 47 & 1 & \\
\hline $0231+385$ & 023428.4 & 384454 & 830 & 58 & 600 & 24 & 470 & 33 & 221 & 7 & 138 & 3 & 84 & 2 & \\
\hline $0231+391$ & 023452.7 & 392342 & 720 & 54 & 340 & 14 & 250 & 31 & 83 & 4 & 22 & 1 & 8 & 1 & \\
\hline $231+405 \mathrm{~A}$ & 023500.2 & 404358 & 1310 & 77 & 940 & 38 & 430 & 33 & 223 & 7 & 64 & 2 & 31 & 1 & \\
\hline $0232+411 \mathrm{~B}$ & 023556.5 & 412316 & 6510 & 328 & 3950 & 158 & 2750 & 88 & 971 & 29 & 353 & 37 & 157 & 3 & $\mathrm{Q}$ \\
\hline $0233+390$ & $\mid \begin{array}{lll}02 & 36 & 50.8\end{array}$ & $\begin{array}{lll}39 & 1303\end{array}$ & 660 & 52 & 420 & 17 & 340 & 32 & 121 & 4 & 35 & 1 & 13 & 1 & \\
\hline $0236+399$ & $\begin{array}{lll}02 & 40 & 09.9\end{array}$ & 401236 & 640 & 51 & 320 & 14 & 250 & 31 & 44 & 1 & 21 & 1 & 4 & 1 & \\
\hline $0236+438$ & $\begin{array}{lll}02 & 40 & 07.5\end{array}$ & 440130 & 3210 & 165 & 1660 & 67 & 1050 & 44 & 314 & 9 & 64 & 2 & 24 & 1 & \\
\hline $0237+389$ & $\begin{array}{lll}02 & 40 & 50.7\end{array}$ & 391133 & 530 & 48 & 280 & 12 & 240 & 31 & 82 & 3 & 22 & 1 & 10 & 1 & \\
\hline $0237+396$ & $\begin{array}{llll}02 & 40 & 55.4\end{array}$ & $\begin{array}{llll}39 & 50 & 37\end{array}$ & 280 & 42 & 120 & 7 & 120 & 30 & 43 & 1 & 18 & 1 & 8 & 1 & \\
\hline
\end{tabular}


Table 2. continued

\begin{tabular}{|c|c|c|c|c|c|c|c|c|c|c|c|c|c|c|c|}
\hline B3 name & $\begin{array}{l}\mathrm{RA}(\mathrm{J} 2000) \\
{\left[\begin{array}{ccc}\mathrm{h} & \mathrm{m} & \mathrm{s}\end{array}\right]}\end{array}$ & 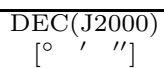 & $\begin{array}{r}S_{151} \\
{[\mathrm{mJy}]}\end{array}$ & $\begin{array}{r}\Delta S_{151} \\
{[\mathrm{mJy}]}\end{array}$ & $\begin{array}{r}S_{327} \\
{[\mathrm{mJy}]}\end{array}$ & $\begin{array}{r}\Delta S_{327} \\
{[\mathrm{mJy}]}\end{array}$ & $\begin{array}{r}S_{408} \\
{[\mathrm{mJy}]}\end{array}$ & $\begin{array}{r}\Delta S_{408} \\
{[\mathrm{mJy}]}\end{array}$ & $\begin{array}{l}S_{1400} \\
{[\mathrm{mJy}]}\end{array}$ & $\begin{array}{r}\Delta S_{1400} \\
{[\mathrm{mJy}]}\end{array}$ & $\begin{array}{l}S_{4850} \\
{[\mathrm{mJy}]}\end{array}$ & $\begin{array}{r}\Delta S_{4850} \\
{[\mathrm{mJy}]}\end{array}$ & $\begin{array}{c}S_{10550} \\
{[\mathrm{mJy}]}\end{array}$ & $\begin{array}{r}\Delta S_{10550} \\
{[\mathrm{mJy}]}\end{array}$ & Id. \\
\hline $0237+435$ & $\begin{array}{llll}02 & 40 & 28.7\end{array}$ & 434757 & 2700 & 141 & 1540 & 62 & 1250 & 48 & 445 & 13 & 149 & 3 & 64 & 2 & $\mathrm{G}$ \\
\hline $0239+395$ & $\begin{array}{lll}02 & 42 & 57.4\end{array}$ & 394409 & 1380 & 80 & 1060 & 47 & 640 & 36 & 234 & 7 & 72 & 2 & 27 & 1 & $\mathrm{~g}$ \\
\hline $0239+397$ & $02 \quad 4254.4$ & $3955 \quad 46$ & 670 & 52 & 450 & 19 & 370 & 32 & 142 & 4 & 50 & 1 & 18 & 1 & $\mathrm{Q}$ \\
\hline $0240+404$ & $\begin{array}{lll}02 & 43 & 37.7\end{array}$ & 404140 & 990 & 64 & 640 & 26 & 400 & 32 & 205 & 6 & 59 & 12 & 33 & 1 & $\mathrm{~g}$ \\
\hline $0241+393 \mathrm{~B}$ & 024431.3 & 393359 & 7060 & 355 & 4860 & 198 & 3230 & 101 & 1220 & 37 & 425 & 44 & 221 & 5 & $\mathrm{~g}$ \\
\hline $0241+395$ & $\mid \begin{array}{lll}02 & 44 & 39.1\end{array}$ & 394342 & 620 & 51 & 360 & 15 & 280 & 31 & 111 & 3 & 34 & 1 & 10 & 1 & \\
\hline $0242+395$ & $02 \quad 45 \quad 28.5$ & 394643 & 600 & 50 & 320 & 14 & 260 & 31 & 87 & 3 & 28 & 1 & 12 & 1 & \\
\hline $0243+439$ & $\mid \begin{array}{lll}02 & 46 & 18.8\end{array}$ & 441144 & 1810 & 99 & 1320 & 53 & 1010 & 43 & 480 & 14 & 159 & 3 & 80 & 2 & \\
\hline $0244+377$ & $\begin{array}{llll}02 & 47 & 31.9\end{array}$ & 375452 & 2980 & 154 & 2010 & 81 & 1730 & 60 & 738 & 22 & 244 & 5 & 100 & 2 & \\
\hline $0246+392$ & 024933.0 & $\begin{array}{lll}39 & 29 & 16\end{array}$ & 1130 & 69 & 630 & 26 & 460 & 33 & 217 & 7 & 83 & 2 & 36 & 1 & \\
\hline $0246+393$ & 025015.6 & 393435 & 12370 & 620 & 7420 & 297 & 6170 & 188 & 2041 & 61 & 585 & 59 & 381 & 8 & $\mathrm{~g}$ \\
\hline $0246+396$ & 024959.0 & 395142 & 480 & 47 & 280 & 12 & 250 & 31 & 95 & 3 & 70 & 7 & 46 & 1 & \\
\hline $0246+428 \mathrm{~A}$ & $\begin{array}{llll}02 & 49 & 30.9\end{array}$ & 430531 & 10190 & 511 & 5940 & 238 & 4970 & 152 & 1720 & 52 & 565 & 57 & 190 & 4 & $\mathrm{~g}$ \\
\hline $0247+395$ & 025020.9 & 394250 & & & 230 & 10 & 120 & 30 & 46 & 1 & 6 & 1 & 5 & 1 & \\
\hline $0247+404$ & $0251 \quad 10.6$ & 404213 & 1740 & 96 & 1120 & 45 & 940 & 41 & 343 & 10 & 103 & 2 & 46 & 1 & $\mathrm{G}$ \\
\hline $0248+392$ & 025157.1 & 392826 & 1240 & 74 & 730 & 30 & 610 & 35 & 216 & 7 & 72 & 2 & 33 & 1 & \\
\hline $0248+396$ & 025156.1 & 395131 & & & 200 & 9 & 130 & 30 & 37 & 1 & 12 & 1 & 6 & 1 & \\
\hline $0248+467$ & $\begin{array}{lll}02 & 51 & 00.1\end{array}$ & 465724 & 6700 & 337 & 3360 & 147 & 2400 & 78 & 630 & 20 & 551 & 12 & 379 & 8 & $\mathrm{~g}$ \\
\hline $0249+383$ & $\mid \begin{array}{lll}02 & 53 & 08.9\end{array}$ & 383525 & 1230 & 73 & 1130 & 45 & 950 & 41 & 669 & 20 & 559 & 11 & 382 & 8 & $\mathrm{Q}$ \\
\hline $0250+384$ & 025352.0 & 384139 & 4560 & 231 & 2250 & 90 & 1640 & 58 & 420 & 13 & 87 & 2 & 30 & 1 & \\
\hline $0250+396$ & 025319.4 & 395344 & 970 & 63 & 530 & 22 & 420 & 33 & 178 & 5 & 63 & 2 & 29 & 1 & \\
\hline $0251+393$ & 025443.7 & 393132 & & & 280 & 12 & 270 & 31 & 231 & 7 & 337 & 7 & 406 & 8 & $\mathrm{~g}$ \\
\hline $0252+385$ & $\mid \begin{array}{lll}02 & 56 & 10.4\end{array}$ & $3842 \quad 32$ & 1140 & 70 & 550 & 22 & 420 & 33 & 115 & 3 & 29 & 1 & 12 & 1 & \\
\hline $0252+388$ & 025601.0 & 390423 & 360 & 44 & 230 & 10 & 200 & 31 & 79 & 2 & 30 & 4 & 14 & 1 & \\
\hline $0252+399$ & 025547.4 & $40 \quad 06 \quad 19$ & & & 130 & 7 & 100 & 30 & 36 & 1 & 12 & 1 & 6 & 1 & \\
\hline $0253+396$ & $\begin{array}{lll}02 & 56 & 47.2\end{array}$ & 395155 & 380 & 44 & 170 & 8 & 140 & 30 & 47 & 2 & 15 & 1 & 5 & 1 & \\
\hline $0254+406$ & 025750.4 & 405033 & 2950 & 153 & 1830 & 73 & 1430 & 52 & 500 & 15 & 149 & 3 & 64 & 2 & $\mathrm{G}$ \\
\hline $0255+460$ & $0258 \quad 30.3$ & 461606 & 3220 & 166 & 2110 & 85 & 1740 & 60 & 719 & 22 & 219 & 5 & 97 & 2 & $\mathrm{Q}$ \\
\hline $0258+435$ & $\mid \begin{array}{lll}03 & 02 & 12.8\end{array}$ & 434247 & 2290 & 121 & 1760 & 71 & 1310 & 49 & 580 & 17 & 203 & 23 & 109 & 2 & $\mathrm{~g}$ \\
\hline $0258+443$ & \begin{tabular}{|lll}
0 & 03 & 0133.7
\end{tabular} & 443027 & 1940 & 105 & 1320 & 53 & 1090 & 44 & 439 & 13 & 139 & 3 & 69 & 2 & \\
\hline $0259+387$ & $\mid \begin{array}{lll}0 & 03 & 00.5\end{array}$ & 385642 & 1000 & 64 & 590 & 24 & 430 & 33 & 164 & 5 & 58 & 2 & 25 & 1 & $\mathrm{~g}$ \\
\hline $0259+391$ & $\begin{array}{llll}0 & 02 & 02 & 30.5\end{array}$ & 392057 & 590 & 50 & 380 & 16 & 280 & 31 & 113 & 3 & 37 & 5 & 14 & 1 & \\
\hline $0700+375$ & $\mid \begin{array}{llll}07 & 04 & 04.2\end{array}$ & 372658 & 3850 & 197 & 2600 & 104 & 2070 & 69 & 661 & 20 & 152 & 3 & 51 & 1 & $\mathrm{G}$ \\
\hline $0700+390$ & $\mid \begin{array}{llll}07 & 04 & 04.4\end{array}$ & 385831 & & & 530 & 22 & 230 & 31 & 177 & 5 & 55 & 11 & 35 & 1 & \\
\hline $0700+398$ & $\begin{array}{lll}07 & 04 & 05.2\end{array}$ & 394826 & & & 130 & 7 & 110 & 30 & 27 & 1 & 6 & 1 & 3 & 1 & \\
\hline $0700+399$ & $\mid \begin{array}{llll}0 & 04 & 24.2\end{array}$ & 394920 & & & 170 & 8 & 130 & 30 & 61 & 2 & 32 & 4 & 17 & 1 & \\
\hline $0701+392$ & $\begin{array}{llll}07 & 04 & 31.3\end{array}$ & 391123 & 1790 & 98 & 1380 & 55 & 1170 & 46 & 506 & 15 & 182 & 4 & 77 & 2 & $\mathrm{Q}$ \\
\hline $0701+397$ & $\mid \begin{array}{lll}07 & 05 & 11.1\end{array}$ & 393748 & & & 80 & 6 & 110 & 30 & 29 & 1 & 11 & 1 & 5 & 1 & \\
\hline $0701+401$ & $\mid \begin{array}{lll}07 & 05 & 21.2\end{array}$ & $\begin{array}{lll}40 & 02 & 27\end{array}$ & 2900 & 150 & 2050 & 82 & 1670 & 58 & 629 & 19 & 200 & 4 & 80 & 2 & \\
\hline $0702+396$ & $\mid \begin{array}{lll}0 & 05 & 51.1\end{array}$ & 393448 & & & 120 & 7 & 130 & 30 & 43 & 1 & 14 & 1 & 8 & 1 & \\
\hline $0703+390$ & $\mid \begin{array}{lll}07 & 06 & 48.4\end{array}$ & 385531 & 560 & 49 & 390 & 16 & 310 & 31 & 123 & 4 & 32 & 1 & 20 & 1 & \\
\hline $0703+426 \mathrm{~A}$ & $\mid \begin{array}{lll}07 & 06 & 42.3\end{array}$ & $42 \quad 32 \quad 11$ & 11040 & 553 & 5960 & 241 & 4300 & 132 & 2297 & 69 & 1078 & 22 & 608 & 12 & $\mathrm{~g}$ \\
\hline $0703+426 \mathrm{~B}$ & $\begin{array}{lll}07 & 07 & 03.3\end{array}$ & 423253 & & & 1880 & 75 & 1040 & 43 & 477 & 15 & 221 & 24 & 71 & 2 & \\
\hline $0703+468$ & 070648.0 & 464756 & 410 & 45 & 1970 & 79 & 1900 & 64 & 1598 & 48 & 637 & 13 & 281 & 6 & \\
\hline $0704+384$ & $\mid \begin{array}{llll}07 & 07 & 32.8\end{array}$ & $\begin{array}{lll}38 & 22 & 13\end{array}$ & 6890 & 347 & 3920 & 157 & 2870 & 91 & 929 & 28 & 288 & 6 & 111 & 2 & $\mathrm{Q}$ \\
\hline $0704+397$ & $\mid \begin{array}{llll}07 & 07 & 32.8\end{array}$ & 394140 & 240 & 42 & 170 & 8 & 140 & 30 & 38 & 1 & 10 & 1 & 5 & 1 & \\
\hline $0704+399$ & $\begin{array}{llll}07 & 07 & 45.0\end{array}$ & 394934 & 250 & 42 & 200 & 9 & 200 & 31 & 72 & 2 & 29 & 1 & 14 & 1 & \\
\hline $0704+418$ & $\mid \begin{array}{lll}0 & 08 & 20.5\end{array}$ & 414852 & 1990 & 107 & 1110 & 45 & 840 & 39 & 273 & 8 & 90 & 2 & 49 & 1 & \\
\hline $0705+398$ & $\mid \begin{array}{llll}07 & 09 & 19.4\end{array}$ & 394833 & 440 & 46 & 230 & 10 & 190 & 31 & 63 & 3 & 16 & 1 & 4 & 1 & \\
\hline $0706+396$ & $\mid \begin{array}{lll}07 & 10 & 01.2\end{array}$ & $3936 \quad 45$ & 890 & 60 & 500 & 21 & 350 & 32 & 127 & 4 & 35 & 1 & 14 & 1 & \\
\hline $0707+380 \mathrm{~A}$ & $\mid \begin{array}{lll}07 & 10 & 39.7\end{array}$ & $\begin{array}{lll}37 & 59 & 17\end{array}$ & 1390 & 80 & 670 & 27 & 430 & 33 & 166 & 5 & 45 & 1 & 18 & 1 & \\
\hline $0708+384$ & $\mid \begin{array}{llll}0 & 11 & 49.8\end{array}$ & 382353 & 980 & 63 & 640 & 26 & 460 & 33 & 142 & 4 & 37 & 1 & 10 & 1 & \\
\hline $0708+388$ & $\begin{array}{lll}07 & 12 & 08.1\end{array}$ & 384825 & 1400 & 81 & 830 & 34 & 660 & 36 & 254 & 8 & 117 & 3 & 56 & 2 & $\mathrm{~g}$ \\
\hline $0709+393$ & $\begin{array}{llll}07 & 13 & 05.6\end{array}$ & 391333 & 600 & 50 & 450 & 19 & 290 & 31 & 185 & 7 & 90 & 13 & 43 & 1 & \\
\hline $0709+398$ & $\begin{array}{llll}07 & 12 & 49.4\end{array}$ & $3948 \quad 45$ & 520 & 48 & 320 & 14 & 260 & 31 & 123 & 4 & 44 & 1 & 21 & 1 & \\
\hline $0709+405$ & $\mid \begin{array}{llll}07 & 13 & 00.8\end{array}$ & 402804 & 1050 & 66 & 640 & 26 & 490 & 33 & 181 & 5 & 63 & 2 & 22 & 1 & \\
\hline $0709+409$ & $\mid \begin{array}{lll}0 & 12 & 30.0\end{array}$ & 405126 & 3090 & 160 & 1670 & 67 & 1290 & 49 & 396 & 12 & 104 & 2 & 41 & 1 & $\mathrm{~g}$ \\
\hline $0710+403$ & $\mid \begin{array}{lll}07 & 14 & 24.4\end{array}$ & 401602 & 1210 & 73 & 660 & 27 & 540 & 34 & 213 & 6 & 69 & 2 & 29 & 1 & $\mathrm{~g}$ \\
\hline $0710+457$ & $\mid \begin{array}{llll}07 & 14 & 31.1\end{array}$ & 454006 & 5810 & 293 & 3710 & 148 & 3000 & 95 & 1242 & 37 & 473 & 10 & 248 & 5 & $\mathrm{~g}$ \\
\hline $0711+397$ & $\begin{array}{lll}07 & 15 & 17.9\end{array}$ & 393954 & 1530 & 86 & 1050 & 42 & 790 & 38 & 222 & 7 & 41 & 1 & 11 & 1 & \\
\hline $0711+399$ & $\begin{array}{lll}07 & 14 & 32.1\end{array}$ & 395247 & & & 90 & 6 & 100 & 30 & 34 & 2 & 13 & 1 & 5 & 1 & \\
\hline $0712+382$ & $\begin{array}{llll}07 & 15 & 50.3\end{array}$ & $\begin{array}{lll}38 & 08 & 07\end{array}$ & 400 & 45 & 510 & 21 & 500 & 34 & 238 & 7 & 56 & 2 & 14 & 1 & \\
\hline $0712+387$ & $\begin{array}{llll}07 & 15 & 34.3\end{array}$ & 383904 & 1370 & 79 & 920 & 37 & 710 & 37 & 239 & 7 & 72 & 2 & 31 & 1 & \\
\hline $0712+388$ & $\begin{array}{llll}07 & 15 & 49.3\end{array}$ & 384836 & 490 & 47 & 330 & 14 & 250 & 31 & 91 & 4 & 38 & 5 & 15 & 1 & \\
\hline $0712+390$ & $\mid \begin{array}{llll}0 & 15 & 37.1\end{array}$ & $\begin{array}{lll}39 & 00 & 05\end{array}$ & & & 250 & 11 & 250 & 31 & 86 & 3 & 35 & 1 & 13 & 1 & \\
\hline $0712+391$ & $\mid \begin{array}{llll}07 & 15 & 29.0\end{array}$ & $\begin{array}{lll}39 & 01 & 09\end{array}$ & & & 710 & 29 & 500 & 34 & 283 & 8 & 78 & 2 & 38 & 1 & \\
\hline $0714+405$ & $\mid \begin{array}{llll}07 & 18 & 11.0\end{array}$ & 402510 & 810 & 57 & 490 & 20 & 400 & 32 & 127 & 4 & 29 & 4 & 14 & 1 & \\
\hline $0717+393$ & $\begin{array}{lll}07 & 20 & 47.5\end{array}$ & $\begin{array}{lll}39 & 16 & 11\end{array}$ & 860 & 59 & 390 & 16 & 310 & 31 & 115 & 3 & 37 & 5 & 13 & 1 & \\
\hline $0720+381$ & $\mid \begin{array}{lll}07 & 24 & 05.9\end{array}$ & $\begin{array}{lll}38 & 03 & 25\end{array}$ & 1930 & 104 & 1200 & 48 & 900 & 40 & 320 & 10 & 70 & 2 & 38 & 1 & $\mathrm{~g}$ \\
\hline $0720+412$ & $\begin{array}{llll}07 & 23 & 40.0\end{array}$ & $\begin{array}{lll}4108 & 18\end{array}$ & 2400 & 126 & 1270 & 51 & 960 & 42 & 236 & 7 & 52 & 2 & 18 & 1 & \\
\hline $0721+394$ & $\begin{array}{lll}07 & 24 & 59.3\end{array}$ & 392122 & 450 & 46 & 290 & 12 & 200 & 31 & 88 & 3 & 39 & 1 & 19 & 1 & \\
\hline $0721+398$ & $\mid \begin{array}{lll}07 & 24 & 26.2\end{array}$ & 394354 & 480 & 47 & 280 & 12 & 220 & 31 & 72 & 3 & 15 & 1 & 6 & 1 & \\
\hline $0722+385$ & $07 \quad 2544.3$ & 382640 & 760 & 55 & 730 & 30 & 590 & 35 & 239 & 7 & 68 & 2 & 27 & 1 & \\
\hline $0722+393 \mathrm{~A}$ & $07 \quad 2550.0$ & $\begin{array}{lll}39 & 17 & 25\end{array}$ & 2840 & 148 & 3570 & 143 & 2910 & 92 & 1115 & 33 & 236 & 5 & 60 & 2 & \\
\hline $0723+397$ & $\mid \begin{array}{lll}07 & 26 & 53.9\end{array}$ & 393948 & 840 & 58 & 510 & 21 & 410 & 32 & 155 & 5 & 48 & 1 & 22 & 1 & \\
\hline $0724+396$ & $\begin{array}{lll}07 & 28 & 10.0\end{array}$ & 393024 & 610 & 50 & 330 & 14 & 240 & 31 & 68 & 2 & 23 & 1 & 10 & 1 & $\mathrm{Q}$ \\
\hline $0726+402$ & 073004.0 & 401102 & 1540 & 87 & 1000 & 40 & 790 & 38 & 312 & 9 & 103 & 2 & 47 & 1 & \\
\hline $0726+431$ & $07 \quad 2948.3$ & 430119 & 3010 & 156 & 1780 & 71 & 1290 & 49 & 425 & 13 & 126 & 3 & 50 & 1 & Q \\
\hline $0727+401$ & 073035.8 & 400123 & 290 & 43 & 390 & 16 & 400 & 32 & 451 & 14 & 144 & 3 & 49 & 1 & \\
\hline
\end{tabular}


Table 2. continued

\begin{tabular}{|c|c|c|c|c|c|c|c|c|c|c|c|c|c|c|c|}
\hline B3 name & $\begin{array}{l}\mathrm{RA}(\mathrm{J} 2000) \\
{\left[\begin{array}{ccc}\mathrm{h} & \mathrm{m} & \mathrm{s}\end{array}\right]}\end{array}$ & $\begin{array}{c}\mathrm{DEC}(\mathrm{J} 2000) \\
{\left[\begin{array}{lll}0 & \prime & \end{array}\right]}\end{array}$ & $\begin{array}{r}S_{151} \\
{[\mathrm{mJy}]}\end{array}$ & $\begin{array}{l}\Delta S_{151} \\
{[\mathrm{mJy}]}\end{array}$ & $\begin{array}{r}S_{327} \\
{[\mathrm{mJy}]}\end{array}$ & $\begin{array}{r}\Delta S_{327} \\
{[\mathrm{mJy}]}\end{array}$ & $\begin{array}{r}S_{408} \\
{[\mathrm{mJy}]}\end{array}$ & $\begin{array}{r}S_{408} \\
{[\mathrm{mJy}]}\end{array}$ & $\begin{array}{l}S_{1400} \\
{[\mathrm{mJy}]}\end{array}$ & $\begin{array}{r}\Delta S_{1400} \\
{[\mathrm{mJy}]}\end{array}$ & $\begin{array}{l}S_{4850} \\
{[\mathrm{mJy}]} \\
\end{array}$ & $\begin{array}{r}\Delta S_{4850} \\
{[\mathrm{mJy}]} \\
\end{array}$ & $\begin{array}{r}S_{10550} \\
{[\mathrm{mJy}]} \\
\end{array}$ & $\begin{array}{r}\Delta S_{10550} \\
{[\mathrm{mJy}]} \\
\end{array}$ & Id. \\
\hline $0728+389$ & 073157.9 & 385126 & 340 & 43 & 280 & 12 & 200 & 31 & 111 & 3 & 35 & 5 & 22 & 1 & \\
\hline $0728+395$ & 073155.3 & 392335 & 640 & 51 & 400 & 17 & 300 & 31 & 92 & 3 & 22 & 1 & 10 & 1 & \\
\hline $0729+391$ & 073320.9 & $\begin{array}{lll}39 & 0506\end{array}$ & 290 & 43 & 280 & 12 & 260 & 31 & 155 & 5 & 100 & 9 & 66 & 2 & $\mathrm{Q}$ \\
\hline $0729+395$ & 073300.9 & 392506 & 600 & 50 & 380 & 16 & 280 & 31 & 105 & 3 & 35 & 5 & 10 & 1 & $\mathrm{~g}$ \\
\hline $0729+397$ & 073258.1 & 393838 & 310 & 43 & 220 & 10 & 150 & 30 & 52 & 3 & 16 & 1 & 7 & 1 & \\
\hline $0729+437$ & 073243.6 & 433540 & 1510 & 85 & 1100 & 44 & 900 & 40 & 387 & 12 & 134 & 3 & 59 & 2 & \\
\hline $0730+396$ & 073349.7 & $\begin{array}{lll}39 & 35 & 19\end{array}$ & 570 & 49 & 290 & 12 & 210 & 31 & 72 & 2 & 26 & 4 & 9 & 1 & \\
\hline $0731+438$ & 073521.9 & 434419 & 5910 & 298 & 3400 & 136 & 2600 & 84 & 774 & 23 & 163 & 3 & 47 & 1 & G \\
\hline $0733+389$ & 073658.9 & $3852 \quad 28$ & 540 & 48 & 270 & 12 & 240 & 31 & 70 & 3 & 29 & 4 & 11 & 1 & \\
\hline $0735+388$ & 073855.4 & 384620 & 570 & 49 & 370 & 15 & 360 & 32 & 124 & 4 & 45 & 1 & 28 & 1 & $\mathrm{~g}$ \\
\hline $0735+390$ & $\begin{array}{lll}07 & 39 & 01.4\end{array}$ & $\begin{array}{lll}38 & 56 & 12\end{array}$ & 1050 & 66 & 600 & 24 & 440 & 33 & 168 & 5 & 52 & 2 & 19 & 1 & $\mathrm{~g}$ \\
\hline $0735+395$ & $\begin{array}{lll}07 & 39 & 04.9\end{array}$ & 392544 & 530 & 48 & 370 & 15 & 280 & 31 & 116 & 4 & 39 & 5 & 20 & 1 & \\
\hline $0736+386$ & $\begin{array}{lll}07 & 40 & 10.8\end{array}$ & $\begin{array}{llll}38 & 33 & 47\end{array}$ & 1180 & 71 & 930 & 37 & 770 & 38 & 295 & 9 & 89 & 2 & 38 & 1 & \\
\hline $0736+398$ & 073928.8 & $\begin{array}{llll}39 & 47 & 09\end{array}$ & 330 & 43 & 220 & 22 & 140 & 30 & 92 & 4 & 27 & 4 & 17 & 1 & \\
\hline $0736+400$ & $\begin{array}{lll}07 & 40 & 06.2\end{array}$ & 395450 & 1030 & 65 & 660 & 27 & 530 & 34 & 226 & 7 & 85 & 2 & 39 & 1 & $\mathrm{~g}$ \\
\hline $0739+396$ & 074252.0 & 392924 & 1210 & 73 & 640 & 26 & 490 & 33 & 145 & 4 & 29 & 1 & 12 & 1 & \\
\hline $0739+397 \mathrm{~A}$ & $\begin{array}{lll}07 & 43 & 09.9\end{array}$ & 393920 & & & 800 & 32 & & & 185 & 6 & 82 & 2 & 20 & 1 & \\
\hline $0739+397 \mathrm{~B}$ & $\begin{array}{lll}07 & 43 & 09.9\end{array}$ & 394132 & & & 640 & 33 & & & 484 & 15 & 289 & 6 & 251 & 5 & Q \\
\hline $0739+398$ & $\begin{array}{lll}07 & 42 & 37.3\end{array}$ & 394435 & 280 & 42 & 210 & 10 & 170 & 30 & 126 & 4 & 110 & 2 & 96 & 2 & $\mathrm{Q}$ \\
\hline $0740+380 \mathrm{C}$ & $0744 \quad 17.5$ & $\begin{array}{lll}37 & 53 \quad 17\end{array}$ & 15590 & 781 & 7460 & 298 & 5550 & 169 & 1347 & 40 & 276 & 6 & 92 & 2 & Q \\
\hline $0740+393$ & 074358.8 & $\begin{array}{lll}39 & 1501\end{array}$ & 4410 & 224 & 2630 & 105 & 2080 & 69 & 660 & 20 & 191 & 4 & 65 & 2 & \\
\hline $0740+474 \mathrm{~B}$ & 074428.0 & $47 \quad 18 \quad 42$ & 3600 & 184 & 2070 & 83 & 1630 & 57 & 610 & 18 & 196 & 4 & 62 & 2 & \\
\hline $0741+396$ & 074430.7 & 393432 & 280 & 42 & 160 & 8 & 140 & 30 & 48 & 3 & 12 & 1 & 5 & 1 & \\
\hline $0741+399$ & 074521.0 & 395103 & 280 & 42 & 180 & 9 & 140 & 30 & 58 & 2 & 24 & 1 & 15 & 1 & \\
\hline $0741+407$ & $\begin{array}{lll}07 & 45 & 18.3\end{array}$ & 403821 & 1170 & 71 & 640 & 26 & 490 & 33 & 157 & 5 & 44 & 1 & 16 & 1 & \\
\hline $0742+376$ & 074540.7 & 373134 & 4190 & 213 & 2600 & 104 & 2100 & 70 & 640 & 19 & 173 & 4 & 85 & 2 & \\
\hline $0742+394$ & $\begin{array}{lll}07 & 46 & 02.4\end{array}$ & 391658 & 1110 & 68 & 600 & 24 & 450 & 33 & 150 & 5 & 41 & 1 & 13 & 1 & \\
\hline $0743+392 \mathrm{~B}$ & $0747 \quad 16.4$ & 390953 & 1710 & 94 & 1110 & 45 & 870 & 40 & 294 & 9 & 87 & 2 & 27 & 1 & \\
\hline $0743+399$ & $\begin{array}{lll}07 & 46 & 30.9\end{array}$ & 395121 & & & 140 & 7 & 100 & 30 & 33 & 1 & 7 & 1 & 1 & 1 & \\
\hline $0744+399$ & $\begin{array}{lll}07 & 4733.2\end{array}$ & 395011 & & & 230 & 10 & 200 & 31 & 94 & 3 & 29 & 4 & 10 & 1 & \\
\hline $0744+464$ & $\begin{array}{lll}07 & 47 & 43.7\end{array}$ & 461858 & 3860 & 197 & 2180 & 87 & 1660 & 58 & 517 & 16 & 143 & 3 & 56 & 2 & $\mathrm{G}$ \\
\hline $0745+397$ & $\begin{array}{lll}07 & 49 & 02.1\end{array}$ & 393851 & & & 140 & 7 & 110 & 30 & 44 & 1 & 17 & 1 & 8 & 1 & \\
\hline $0745+398$ & $0748 \quad 41.9$ & 394107 & 1030 & 65 & 590 & 24 & 410 & 32 & 130 & 4 & 34 & 1 & 13 & 1 & \\
\hline $0746+399$ & 075023.1 & $3951 \quad 13$ & 1770 & 97 & 1030 & 41 & 750 & 37 & 232 & 7 & 68 & 2 & 24 & 1 & \\
\hline $0747+398 \mathrm{~A}$ & 075025.8 & 394134 & 2130 & 114 & 1300 & 52 & 970 & 42 & 346 & 10 & 98 & 2 & 38 & 1 & \\
\hline $0747+398 \mathrm{~B}$ & 075120.4 & 394406 & 1960 & 106 & 1130 & 45 & 880 & 40 & 292 & 9 & 90 & 2 & 24 & 1 & \\
\hline $0748+413 \mathrm{~B}$ & 075219.9 & 411552 & 2110 & 113 & 1120 & 45 & 830 & 39 & 236 & 7 & 75 & 2 & 24 & 1 & \\
\hline $0749+398$ & 075238.4 & 394416 & 2160 & 115 & 1330 & 53 & 1020 & 43 & 306 & 9 & 60 & 2 & 18 & 1 & \\
\hline $0749+399$ & 075251.6 & 394648 & & & 180 & 9 & 120 & 30 & 59 & 2 & 18 & 1 & 9 & 1 & \\
\hline $0750+400$ & 075421.3 & 395551 & 1040 & 66 & 610 & 25 & 440 & 33 & 135 & 4 & 35 & 1 & 16 & 1 & \\
\hline $0750+402$ & 075340.7 & 400450 & 1180 & 71 & 600 & 24 & 450 & 33 & 136 & 4 & 34 & 1 & 12 & 1 & \\
\hline $0751+392$ & 075427.4 & $\begin{array}{lll}39 & 08 & 11\end{array}$ & 1660 & 92 & 1010 & 41 & 800 & 38 & 284 & 9 & 96 & 2 & 46 & 1 & \\
\hline $0752+398$ & 075531.0 & 394321 & 390 & 45 & 270 & 12 & 210 & 31 & 69 & 2 & 26 & 4 & 10 & 1 & \\
\hline $0753+383$ & 075651.2 & 380948 & 3580 & 183 & 2020 & 81 & 1510 & 54 & 449 & 13 & 102 & 2 & 50 & 1 & \\
\hline $0753+391$ & 075627.1 & 390125 & 1190 & 72 & 620 & 25 & 480 & 33 & 170 & 5 & 54 & 2 & 36 & 1 & $\mathrm{~g}$ \\
\hline $0754+394$ & 075803.0 & 392043 & 350 & 44 & 150 & 8 & 130 & 30 & 32 & 1 & 9 & 1 & 5 & 1 & $\mathrm{Q}$ \\
\hline $0754+396$ & 075808.9 & 392927 & 3210 & 165 & 1990 & 80 & 1530 & 55 & 548 & 16 & 148 & 3 & 49 & 1 & G \\
\hline $0754+397$ & 075759.1 & 393638 & & & 210 & 22 & 170 & 30 & 25 & 2 & 3 & 1 & 3 & 1 & $\mathrm{~g}$ \\
\hline $0755+379 \mathrm{~B}$ & 075828.1 & $\begin{array}{lll}37 & 47 & 11\end{array}$ & 9220 & 463 & 6190 & 248 & 5420 & 165 & 2652 & 80 & 1260 & 126 & 732 & 15 & $\mathrm{~g}$ \\
\hline $0756+377$ & 075947.1 & 373850 & 8370 & 420 & 5550 & 222 & 4540 & 139 & 1631 & 49 & 469 & 9 & 172 & 4 & \\
\hline $0756+383$ & 075952.6 & $\begin{array}{llll}38 & 14 & 23\end{array}$ & 1860 & 101 & 1240 & 50 & 970 & 42 & 478 & 14 & 174 & 4 & 64 & 2 & $\mathrm{~g}$ \\
\hline $0756+406$ & $\begin{array}{lll}08 & 00 & 16.0\end{array}$ & 402955 & 1100 & 68 & 650 & 26 & 500 & 34 & 202 & 6 & 73 & 2 & 42 & 1 & Q \\
\hline $0757+395$ & $\begin{array}{lll}08 & 00 & 52.9\end{array}$ & 392436 & 640 & 51 & 370 & 15 & 290 & 31 & 156 & 6 & 58 & 12 & 27 & 1 & $\mathrm{~g}$ \\
\hline $0757+399$ & $\begin{array}{lll}08 & 00 & 32.8\end{array}$ & $\begin{array}{lll}39 & 47 & 07\end{array}$ & 430 & 45 & 420 & 17 & 320 & 31 & 117 & 4 & 29 & 4 & 14 & 1 & \\
\hline $0759+392$ & $\begin{array}{lll}08 & 02 & 23.6\end{array}$ & 390435 & 450 & 46 & 240 & 11 & 200 & 31 & 72 & 2 & 26 & 1 & 12 & 1 & \\
\hline $0759+397$ & $\begin{array}{lll}08 & 03 & 02.5\end{array}$ & 393636 & 720 & 54 & 490 & 20 & 390 & 32 & 145 & 4 & 35 & 1 & 15 & 1 & \\
\hline $0800+399$ & $\begin{array}{lll}08 & 04 & 01.1\end{array}$ & 395005 & 530 & 48 & 310 & 13 & 220 & 31 & 100 & 3 & 38 & 5 & 20 & 1 & $\mathrm{~b}$ \\
\hline $0800+472$ & $\begin{array}{llll}08 & 04 & 13.9\end{array}$ & 470442 & 3610 & 185 & 2570 & 105 & 2060 & 69 & 896 & 27 & 322 & 7 & 171 & 4 & $\mathrm{~b}$ \\
\hline $0801+394$ & $\begin{array}{lll}08 & 04 & 38.7\end{array}$ & 392034 & 360 & 44 & 180 & 9 & 180 & 30 & 55 & 2 & 17 & 1 & 6 & 1 & \\
\hline $0801+399$ & $\begin{array}{lll}08 & 04 & 32.9\end{array}$ & 394956 & 550 & 49 & 260 & 11 & 180 & 30 & 41 & 1 & 12 & 1 & $<2$ & & \\
\hline $0801+401$ & $\begin{array}{lll}08 & 05 & 20.8\end{array}$ & 395743 & 880 & 59 & 690 & 28 & 580 & 35 & 310 & 9 & 139 & 3 & 64 & 2 & \\
\hline $0802+398$ & $\begin{array}{lll}08 & 05 & 57.9\end{array}$ & 394044 & 1050 & 66 & 550 & 22 & 410 & 32 & 144 & 4 & 42 & 1 & 22 & 1 & Q \\
\hline $0802+406$ & $\begin{array}{lll}08 & 05 & 31.3\end{array}$ & 402760 & 940 & 62 & 620 & 25 & 490 & 33 & 205 & 6 & 71 & 2 & 29 & 1 & \\
\hline $0803+426$ & $\begin{array}{lll}08 & 06 & 42.4\end{array}$ & 423250 & & & 570 & 23 & 420 & 33 & 152 & 5 & 41 & 1 & 10 & 1 & $\mathrm{~g}$ \\
\hline $0803+427$ & $\begin{array}{lll}08 & 07 & 07.3\end{array}$ & 423437 & & & 740 & 30 & 580 & 35 & 208 & 6 & 38 & 1 & 6 & 1 & $\mathrm{~g}$ \\
\hline $0804+399$ & $\begin{array}{lll}08 & 07 & 46.4\end{array}$ & 394639 & 320 & 43 & 160 & 8 & 150 & 30 & 54 & 3 & 12 & 1 & 6 & 1 & $\mathrm{~g}$ \\
\hline $0805+391$ & $\begin{array}{lll}08 & 08 & 22.3\end{array}$ & $\begin{array}{lll}39 & 01 & 01\end{array}$ & 800 & 57 & 280 & 23 & 230 & 31 & 86 & 3 & 38 & 5 & 19 & 1 & $\mathrm{~g}$ \\
\hline $0805+392$ & $\begin{array}{lll}08 & 08 & 44.4\end{array}$ & 390648 & & & 380 & 16 & 230 & 31 & 70 & 3 & 23 & 1 & 11 & 1 & \\
\hline $0805+406$ & $\begin{array}{llll}08 & 09 & 03.1\end{array}$ & 403256 & 2810 & 146 & 1700 & 68 & 1330 & 50 & 534 & 16 & 201 & 4 & 110 & 2 & b \\
\hline $0806+399$ & $\begin{array}{lll}08 & 09 & 32.5\end{array}$ & 394929 & 810 & 57 & 620 & 25 & 490 & 33 & 247 & 7 & 90 & 2 & 37 & 1 & \\
\hline $0806+426$ & $\begin{array}{lll}08 & 10 & 03.6\end{array}$ & 422806 & 11350 & 569 & 7220 & 289 & 5680 & 173 & 2098 & 63 & 633 & 13 & 179 & 4 & $\mathrm{G}$ \\
\hline $0807+399$ & $\begin{array}{lll}08 & 10 & 43.1\end{array}$ & $\begin{array}{lll}39 & 50 & 10\end{array}$ & 630 & 51 & 290 & 12 & 230 & 31 & 76 & 3 & 24 & 1 & 13 & 1 & \\
\hline $0809+404$ & $\begin{array}{lll}08 & 12 & 53.1\end{array}$ & 401900 & 3470 & 178 & 2660 & 106 & 2310 & 76 & 1072 & 32 & 402 & 8 & 195 & 4 & $\mathrm{~g}$ \\
\hline $0810+460 \mathrm{~B}$ & $\begin{array}{lll}08 & 14 & 30.3\end{array}$ & 455640 & 10260 & 515 & 5370 & 215 & 4080 & 126 & 1119 & 34 & 280 & 6 & 113 & 2 & $\mathrm{~g}$ \\
\hline $0811+388$ & $\begin{array}{lll}08 & 15 & 12.4\end{array}$ & 384043 & 3120 & 161 & 1870 & 75 & 1510 & 54 & 532 & 16 & 148 & 3 & 52 & 1 & $\mathrm{~g}$ \\
\hline $0811+391$ & $\begin{array}{lll}08 & 14 & 27.0\end{array}$ & 385924 & 740 & 54 & 420 & 17 & 270 & 31 & 114 & 5 & 29 & 6 & 10 & 1 & $\mathrm{G}$ \\
\hline $0812+382$ & $\begin{array}{lll}08 & 16 & 01.6\end{array}$ & 380415 & 1810 & 99 & 1200 & 48 & 970 & 42 & 436 & 13 & 163 & 3 & 91 & 2 & $\mathrm{~g}$ \\
\hline $0812+398$ & 081559.0 & $3943 \quad 31$ & & & 80 & 6 & 100 & 30 & 28 & 1 & 8 & 1 & 3 & 1 & \\
\hline $0812+399$ & $\begin{array}{llll}08 & 15 & 58.4 \\
\end{array}$ & $\begin{array}{l}3949 \quad 12 \\
\end{array}$ & & & 80 & 6 & 120 & 30 & 31 & 1 & 10 & 1 & 5 & 1 & \\
\hline
\end{tabular}


Table 2. continued

\begin{tabular}{|c|c|c|c|c|c|c|c|c|c|c|c|c|c|c|c|}
\hline B3 name & $\begin{array}{l}\text { RA(J2000) } \\
{\left[\begin{array}{lll}\mathrm{h} & \mathrm{m} & \mathrm{s}\end{array}\right]}\end{array}$ & 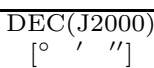 & $\begin{array}{r}S_{151} \\
{[\mathrm{mJy}]}\end{array}$ & $\begin{array}{l}\Delta S_{151} \\
{[\mathrm{mJy}]}\end{array}$ & $\begin{array}{r}S_{327} \\
{[\mathrm{mJy}]}\end{array}$ & $\begin{array}{r}\Delta S_{327} \\
{[\mathrm{mJy}]}\end{array}$ & $\begin{array}{r}S_{408} \\
{[\mathrm{mJy}]}\end{array}$ & $\begin{array}{r}\Delta S_{408} \\
{[\mathrm{mJy}]}\end{array}$ & $\begin{array}{l}S_{1400} \\
{[\mathrm{mJy}]}\end{array}$ & $\begin{array}{r}\Delta S_{1400} \\
{[\mathrm{mJy}]}\end{array}$ & $\begin{array}{l}S_{4850} \\
{[\mathrm{mJy}]}\end{array}$ & $\begin{array}{r}\Delta S_{4850} \\
{[\mathrm{mJy}]}\end{array}$ & $\begin{array}{r}S_{10550} \\
{[\mathrm{mJy}]}\end{array}$ & $\begin{array}{r}\Delta S_{10550} \\
{[\mathrm{mJy}]}\end{array}$ & Id. \\
\hline $0812+406$ & \begin{tabular}{|llll} 
& 16 & 08.3 \\
\end{tabular} & 402918 & 1610 & 90 & 910 & 37 & 700 & 37 & 245 & 7 & 70 & 2 & 32 & 1 & \\
\hline $0813+381$ & $08 \quad 1651.9$ & 375904 & 1030 & 65 & 550 & 22 & 440 & 33 & 145 & 4 & 35 & 1 & 13 & 1 & \\
\hline $0813+393$ & $08 \quad 1653.6$ & $3911 \quad 14$ & 450 & 46 & 250 & 11 & 210 & 31 & 77 & 2 & 29 & 4 & 22 & 1 & $\mathrm{~g}$ \\
\hline $0813+398$ & $\begin{array}{llll}08 & 16 & 46.4\end{array}$ & 394409 & 720 & 54 & 440 & 18 & 310 & 31 & 141 & 4 & 42 & 5 & 17 & 1 & $\mathrm{~g}$ \\
\hline $0814+383$ & $\begin{array}{llll}08 & 17 & 19.5\end{array}$ & 381128 & 1270 & 75 & 670 & 27 & 560 & 34 & 173 & 5 & 49 & 1 & 18 & 1 & \\
\hline $0814+425$ & $\begin{array}{llll}08 & 18 & 16.0\end{array}$ & $42 \quad 2246$ & 1510 & 85 & 1780 & 71 & 1420 & 52 & 1120 & 34 & 1867 & 37 & 1076 & 22 & BL \\
\hline $0814+441$ & $08 \quad 1735.5$ & 435935 & 2150 & 115 & 1160 & 47 & 840 & 39 & 282 & 8 & 85 & 2 & 39 & 1 & \\
\hline $0815+397$ & $\begin{array}{llll}08 & 18 & 44.6\end{array}$ & $3938 \quad 16$ & 430 & 45 & 210 & 10 & 160 & 30 & 48 & 1 & 10 & 1 & 4 & 1 & \\
\hline $0818+472 \mathrm{~A}$ & 082133.6 & 470236 & 10010 & 502 & 5960 & 238 & 4680 & 144 & 1753 & 53 & 616 & 12 & 197 & 4 & $\mathrm{~g}$ \\
\hline $0819+397$ & 082229.5 & 393548 & 440 & 46 & 230 & 10 & 190 & 31 & 66 & 3 & 24 & 4 & 8 & 1 & \\
\hline $0820+392$ & 082324.2 & 390626 & 1190 & 72 & 680 & 28 & 580 & 35 & 185 & 6 & 58 & 2 & 26 & 1 & $\mathrm{~g}$ \\
\hline $0820+431$ & 082327.5 & 425657 & 7120 & 358 & 3960 & 158 & 3060 & 97 & 947 & 28 & 251 & 5 & 103 & 2 & $\mathrm{~g}$ \\
\hline $0821+394$ & 082455.6 & $39 \quad 1643$ & 4410 & 224 & 3450 & 138 & 2590 & 83 & 1489 & 45 & 1138 & 23 & 1741 & 35 & $\mathrm{Q}$ \\
\hline $0821+395$ & 082450.4 & $\begin{array}{lll}39 & 23 & 32\end{array}$ & & & 250 & 11 & 130 & 30 & 56 & 2 & 20 & 1 & $<5$ & & \\
\hline $0821+447$ & $\begin{array}{lll}08 & 25 & 17.6\end{array}$ & 443628 & 5120 & 259 & 2880 & 117 & 2260 & 74 & 726 & 22 & 223 & 5 & 97 & 2 & Q \\
\hline $0822+390$ & $08 \quad 2530.1$ & $38 \quad 52 \quad 14$ & 560 & 49 & 320 & 14 & 220 & 31 & 108 & 3 & 33 & 5 & 16 & 1 & \\
\hline $0822+394$ & 082523.7 & $39 \quad 1945$ & & & 1590 & 64 & 1760 & 61 & 1180 & 35 & 300 & 6 & 127 & 3 & G \\
\hline $0822+398$ & $\begin{array}{lll}08 & 25 & 31.2\end{array}$ & 394105 & 340 & 43 & 140 & 7 & 150 & 30 & 25 & 2 & 7 & 1 & 4 & 1 & \\
\hline $0823+384 \mathrm{~B}$ & 082713.1 & $38 \quad 1958$ & 1120 & 69 & 530 & 22 & 410 & 32 & 142 & 4 & 45 & 1 & 22 & 1 & $\mathrm{~g}$ \\
\hline $0823+399$ & 082654.1 & $3948 \quad 44$ & 250 & 42 & 150 & 8 & 120 & 30 & 66 & 2 & 30 & 1 & 18 & 1 & $\mathrm{~g}$ \\
\hline $0824+397$ & $\begin{array}{llll}08 & 28 & 07.0\end{array}$ & 393542 & 670 & 52 & 320 & 14 & 270 & 31 & 86 & 3 & 32 & 1 & 15 & 1 & $\mathrm{Q}$ \\
\hline $0827+378$ & $\begin{array}{llll}08 & 31 & 10.1\end{array}$ & $\begin{array}{lll}37 & 42 \quad 10\end{array}$ & 9250 & 464 & 6200 & 248 & 5170 & 158 & 2270 & 68 & 936 & 19 & 494 & 10 & $\mathrm{Q}$ \\
\hline $0827+387$ & 083025.2 & 383751 & 1280 & 75 & 740 & 30 & 600 & 35 & 182 & 5 & 44 & 1 & 15 & 1 & \\
\hline $0827+395$ & $\begin{array}{llll}08 & 30 & 19.2\end{array}$ & 392347 & 290 & 43 & 150 & 8 & 110 & 30 & 58 & 2 & 26 & 1 & 13 & 1 & $\mathrm{~g}$ \\
\hline $0827+458$ & 083035.8 & 454330 & 6260 & 316 & 3050 & 122 & 2360 & 77 & 619 & 19 & 134 & 3 & 48 & 1 & \\
\hline $0828+381$ & 083151.0 & 375632 & 960 & 62 & 560 & 23 & 460 & 33 & 156 & 5 & 40 & 1 & 19 & 1 & $\mathrm{~g}$ \\
\hline $0829+395$ & $0833 \quad 15.4$ & 392118 & 1500 & 85 & 850 & 34 & 630 & 35 & 226 & 7 & 64 & 2 & 30 & 1 & \\
\hline $0829+425$ & 083248.5 & $42 \quad 2459$ & 1500 & 85 & 1030 & 41 & 820 & 39 & 458 & 14 & 229 & 5 & 135 & 3 & $\mathrm{Q}$ \\
\hline $0831+393$ & 083455.0 & 391052 & 330 & 43 & 240 & 11 & 210 & 31 & 76 & 2 & 27 & 4 & 15 & 1 & \\
\hline $0831+399$ & 083433.8 & 394453 & 690 & 53 & 360 & 15 & 250 & 31 & 103 & 3 & 27 & 4 & 17 & 1 & \\
\hline $0832+395$ & 083531.4 & 392246 & 580 & 49 & 300 & 13 & 200 & 31 & 115 & 3 & 74 & 7 & 36 & 1 & \\
\hline $0832+399$ & 083539.1 & 394440 & & & 100 & 6 & 110 & 30 & 34 & 1 & 12 & 1 & 6 & 1 & \\
\hline $0834+399$ & 083726.0 & 394723 & & & 140 & 7 & 100 & 30 & 43 & 1 & 16 & 1 & 8 & 1 & \\
\hline $0834+450 \mathrm{~A}$ & 083752.6 & 445023 & 7840 & 394 & 4660 & 186 & 3450 & 108 & 1510 & 45 & 535 & 54 & 301 & 6 & $\mathrm{~g}$ \\
\hline $0836+399$ & $\begin{array}{llll}08 & 40 & 11.8\end{array}$ & 394350 & 240 & 42 & 140 & 7 & 120 & 30 & 37 & 2 & 12 & 1 & 8 & 1 & \\
\hline $0836+402$ & $\begin{array}{llll}08 & 40 & 11.4\end{array}$ & 400359 & 4280 & 218 & 2370 & 95 & 1870 & 64 & 612 & 18 & 184 & 4 & 84 & 2 & $\mathrm{~g}$ \\
\hline $0836+426$ & 083956.6 & 422755 & 2020 & 109 & 1350 & 54 & 1120 & 45 & 526 & 16 & 331 & 7 & 208 & 4 & $\mathrm{Q}$ \\
\hline $0837+399$ & $\begin{array}{lll}08 & 41 & 07.9\end{array}$ & 394448 & 320 & 43 & 180 & 9 & 160 & 30 & 53 & 2 & 17 & 1 & 6 & 1 & \\
\hline $0838+396$ & $0842 \quad 10.5$ & $\begin{array}{lll}39 & 27 & 12\end{array}$ & 240 & 42 & 160 & 8 & 150 & 30 & 54 & 2 & 21 & 1 & 8 & 1 & \\
\hline $0840+400$ & 084406.8 & 395301 & 280 & 42 & 610 & 25 & 580 & 35 & 144 & 4 & 14 & 1 & 2 & 1 & \\
\hline $0840+424 \mathrm{~A}$ & 084331.6 & $42 \quad 1530$ & 1510 & 85 & 2530 & 101 & 2280 & 75 & 1420 & 43 & 552 & 11 & 272 & 6 & \\
\hline $0841+386$ & 084429.0 & 383055 & 730 & 54 & 540 & 22 & 490 & 33 & 430 & 13 & 214 & 4 & 245 & 5 & \\
\hline $0841+397$ & 084421.7 & 393410 & 360 & 44 & 160 & 8 & 130 & 30 & 24 & 1 & 3 & 1 & $<3$ & & \\
\hline $0841+403$ & 084455.9 & $40 \quad 08 \quad 14$ & 2720 & 142 & 1460 & 59 & 1120 & 45 & 401 & 12 & 128 & 3 & 55 & 1 & $\mathrm{~g}$ \\
\hline $0841+407$ & 084508.6 & 403116 & 870 & 59 & 450 & 19 & 400 & 32 & 163 & 5 & 66 & 7 & 38 & 1 & \\
\hline $0842+401$ & 084603.6 & 395656 & 1290 & 76 & 750 & 30 & 570 & 35 & 195 & 6 & 67 & 2 & 23 & 1 & \\
\hline $0843+425$ & $0847 \quad 18.6$ & $\begin{array}{lll}42 & 23 & 39\end{array}$ & 2420 & 127 & 1250 & 50 & 910 & 41 & 353 & 11 & 107 & 15 & 51 & 1 & $\mathrm{~g}$ \\
\hline $0844+396$ & 084750.6 & 393001 & 220 & 41 & 150 & 8 & 110 & 30 & 41 & 1 & 12 & 1 & 7 & 1 & \\
\hline $0847+406$ & 085040.9 & $40 \quad 2914$ & 1480 & 84 & 720 & 29 & 480 & 33 & 122 & 4 & 32 & 1 & 13 & 1 & \\
\hline $0849+394$ & 085239.0 & 391906 & 250 & 42 & 120 & 7 & 110 & 30 & 37 & 1 & 13 & 1 & 6 & 1 & \\
\hline $0849+424$ & $0852 \quad 34.1$ & $42 \quad 15 \quad 27$ & 3220 & 166 & 1730 & 69 & 1310 & 49 & 458 & 14 & 139 & 3 & 57 & 2 & $\mathrm{Q}$ \\
\hline $0850+383$ & 085353.6 & $38 \quad 11 \quad 15$ & 1240 & 74 & 530 & 22 & 400 & 32 & 116 & 4 & 27 & 4 & 12 & 1 & \\
\hline $0852+384$ & 085555.2 & $\begin{array}{llll}38 & 13 & 32\end{array}$ & 1060 & 66 & 580 & 24 & 440 & 33 & 175 & 5 & 51 & 1 & 24 & 1 & \\
\hline $0854+399 \mathrm{~B}$ & $\begin{array}{llll}08 & 57 & 42.8\end{array}$ & 394536 & 3170 & 163 & 1780 & 71 & 1460 & 53 & 501 & 16 & 140 & 17 & 71 & 2 & G \\
\hline $0855+397$ & $0858 \quad 15.5$ & 393055 & 390 & 45 & 130 & 7 & 100 & 30 & 44 & 2 & 12 & 1 & 4 & 1 & \\
\hline $0855+419$ & 085900.5 & $4143 \quad 19$ & 2290 & 121 & 1210 & 49 & 960 & 42 & 285 & 9 & 65 & 2 & 25 & 1 & \\
\hline $0856+397$ & 085938.6 & 393022 & 470 & 46 & 180 & 9 & 120 & 30 & 49 & 2 & 14 & 1 & 10 & 1 & \\
\hline $0856+406$ & 085959.5 & 402434 & 3010 & 156 & 1460 & 59 & 1070 & 44 & 241 & 7 & 41 & 1 & 10 & 1 & G \\
\hline $0857+391$ & $\begin{array}{llll}09 & 00 & 53.9\end{array}$ & $\begin{array}{lll}38 & 56 & 13\end{array}$ & 2710 & 141 & 1570 & 63 & 1280 & 49 & 508 & 15 & 188 & 4 & 86 & 2 & $\mathrm{~g}$ \\
\hline $0857+402$ & $\begin{array}{llll}09 & 00 & 29.7\end{array}$ & 400458 & 1230 & 73 & 710 & 29 & 560 & 34 & 215 & 6 & 57 & 2 & 22 & 1 & \\
\hline $0858+386$ & 090132.2 & $\begin{array}{llll}38 & 27 & 11\end{array}$ & 1510 & 85 & 1060 & 43 & 740 & 37 & 327 & 10 & 118 & 3 & 58 & 2 & \\
\hline $0858+388$ & $\begin{array}{llll}09 & 01 & 14.8\end{array}$ & 384208 & 1270 & 75 & 550 & 22 & 420 & 33 & 116 & 4 & 29 & 1 & 10 & 1 & \\
\hline $0858+452$ & $\begin{array}{lll}09 & 02 & 15.7\end{array}$ & 450053 & 4730 & 240 & 2770 & 111 & 2170 & 72 & 839 & 25 & 266 & 5 & 104 & 2 & \\
\hline $0859+470$ & $\begin{array}{llll}09 & 03 & 03.9\end{array}$ & 465103 & 4520 & 230 & 3430 & 137 & 2810 & 89 & 1783 & 53 & 1292 & 26 & 933 & 19 & Q \\
\hline $0900+380 \mathrm{~B}$ & 090400.0 & 375232 & 1030 & 65 & 530 & 22 & 450 & 33 & 159 & 5 & 49 & 1 & 16 & 1 & \\
\hline $0900+389$ & 090411.7 & $\begin{array}{lll}38 & 46 & 29\end{array}$ & 1530 & 86 & 680 & 28 & 550 & 34 & 177 & 5 & 53 & 2 & 24 & 1 & \\
\hline $0900+395$ & 090325.6 & 391841 & 440 & 46 & 190 & 9 & 150 & 30 & 40 & 1 & 10 & 1 & 9 & 1 & \\
\hline $0900+428$ & 090415.7 & 423805 & 5020 & 254 & 3010 & 120 & 2650 & 85 & 1253 & 38 & 939 & 19 & 451 & 9 & $\mathrm{~g}$ \\
\hline $0902+383$ & 090528.0 & 380726 & 1740 & 96 & 870 & 35 & 700 & 37 & 224 & 7 & 69 & 2 & 28 & 1 & \\
\hline $0902+384$ & 090513.0 & 381434 & 1600 & 89 & 840 & 34 & 610 & 35 & 163 & 5 & 31 & 1 & 11 & 1 & \\
\hline $0902+414$ & $\begin{array}{lll}09 & 06 & 02.5\end{array}$ & $41 \quad 1629$ & 4690 & 238 & 2600 & 104 & 2030 & 68 & 813 & 24 & 294 & 6 & 133 & 3 & $\mathrm{~g}$ \\
\hline $0902+416$ & $\begin{array}{llll}09 & 05 & 22.2\end{array}$ & $41 \quad 2839$ & 1740 & 96 & 1070 & 43 & 930 & 41 & 503 & 15 & 191 & 4 & 104 & 2 & \\
\hline $0903+428$ & 090626.2 & 423905 & 3140 & 162 & 1490 & 60 & 1200 & 47 & 292 & 9 & 69 & 2 & 27 & 1 & G \\
\hline $0904+386$ & $\begin{array}{llll}09 & 07 & 45.3\end{array}$ & $38 \quad 2739$ & 1120 & 69 & 540 & 22 & 430 & 33 & 161 & 5 & 83 & 2 & 27 & 1 & Q \\
\hline $0904+396$ & 090738.5 & 392429 & 720 & 54 & 340 & 14 & 260 & 31 & 76 & 2 & 13 & 1 & 6 & 1 & \\
\hline $0904+399$ & 090727.6 & 394420 & & & 190 & 9 & 160 & 30 & 87 & 3 & 48 & 5 & 36 & 1 & $\mathrm{~g}$ \\
\hline $0904+417 \mathrm{~B}$ & 090733.1 & 413442 & 9020 & 453 & 5940 & 239 & 4090 & 126 & 1467 & 44 & 456 & 9 & 200 & 4 & $\mathrm{~g}$ \\
\hline $0905+380 \mathrm{~A}$ & 090850.6 & 374818 & 15940 & 798 & 8970 & 359 & 7090 & 215 & 2102 & 63 & 469 & 9 & 161 & 3 & $\mathrm{G}$ \\
\hline $0905+399$ & $\begin{array}{llll}09 & 08 & 17.8\end{array}$ & $3943 \quad 15$ & 2820 & 147 & 1320 & 53 & 940 & 41 & 253 & 8 & 40 & 11 & 17 & 1 & G \\
\hline
\end{tabular}


Table 2. continued

\begin{tabular}{|c|c|c|c|c|c|c|c|c|c|c|c|c|c|c|c|}
\hline B3 name & $\begin{array}{l}\mathrm{RA}(\mathrm{J} 2000) \\
{\left[\begin{array}{ccc}\mathrm{h} & \mathrm{m} & \mathrm{s}\end{array}\right]}\end{array}$ & $\begin{array}{c}\mathrm{DEC}(\mathrm{J} 2000) \\
{\left[\begin{array}{lll} & 1 & \end{array}\right]}\end{array}$ & $\begin{array}{r}S_{151} \\
{[\mathrm{mJy}]}\end{array}$ & $\begin{array}{r}\Delta S_{151} \\
{[\mathrm{mJy}]} \\
\end{array}$ & $\begin{array}{r}S_{327} \\
{[\mathrm{mJy}]}\end{array}$ & $\begin{array}{r}S_{327} \\
{[\mathrm{mJy}]}\end{array}$ & $\begin{array}{r}S_{408} \\
{[\mathrm{mJy}]}\end{array}$ & $\begin{array}{r}S_{408} \\
{[\mathrm{mJy}]} \\
\end{array}$ & $\begin{array}{l}S_{1400} \\
{[\mathrm{mJy}]}\end{array}$ & $\begin{array}{r}\Delta S_{1400} \\
{[\mathrm{mJy}]}\end{array}$ & $\begin{array}{l}S_{4850} \\
{[\mathrm{mJy}]}\end{array}$ & $\begin{array}{r}\Delta S_{4850} \\
{[\mathrm{mJy}]} \\
\end{array}$ & $\begin{array}{r}S_{10550} \\
{[\mathrm{mJy}]} \\
\end{array}$ & $\begin{array}{r}S_{10550} \\
{[\mathrm{mJy}]}\end{array}$ & Id. \\
\hline $0906+383$ & $\begin{array}{lllll}0 & 10 & 02.8\end{array}$ & $38 \quad 06 \quad 17$ & 1510 & 85 & 770 & 31 & 610 & 35 & 197 & 6 & 56 & 2 & 20 & 1 & \\
\hline $0906+421$ & 090945.6 & 415720 & 3370 & 173 & 1620 & 65 & 1290 & 49 & 267 & 8 & 30 & 1 & 5 & 1 & $\mathrm{~g}$ \\
\hline $0906+430$ & 090933.5 & 425346 & 27750 & 1388 & 15160 & 606 & 11900 & 358 & 4221 & 127 & 1541 & 31 & 1128 & 23 & $\mathrm{Q}$ \\
\hline $0907+381$ & $09 \quad 1054.2$ & 375915 & 560 & 49 & 510 & 21 & 430 & 33 & 285 & 9 & 134 & 3 & 107 & 2 & Q \\
\hline $0908+380 \mathrm{~B}$ & $\begin{array}{llll}09 & 11 & 48.5\end{array}$ & $\begin{array}{lll}37 & 50 & 18\end{array}$ & & & 550 & 22 & & & 159 & 5 & 66 & 2 & 26 & 1 & $\mathrm{G}$ \\
\hline $0908+380 \mathrm{C}$ & \begin{tabular}{|lll}
09 & 12 & 02.9
\end{tabular} & 375134 & 13140 & 658 & 6500 & 260 & 4580 & 141 & 1124 & 34 & 294 & 6 & 109 & 2 & $\mathrm{G}$ \\
\hline $0909+395$ & $\begin{array}{llll}09 & 12 & 40.7\end{array}$ & 392245 & 250 & 42 & 110 & 6 & 100 & 30 & 41 & 1 & 13 & 1 & 6 & 1 & \\
\hline $0909+432$ & 091300.7 & 430521 & 3360 & 173 & 1600 & 64 & 1210 & 47 & 366 & 11 & 93 & 2 & 36 & 1 & \\
\hline $910+392$ & $\begin{array}{lll}09 & 13 & 51.7\end{array}$ & $\begin{array}{lll}39 & 02 & 10\end{array}$ & 990 & 64 & 520 & 21 & 410 & 32 & 143 & 4 & 44 & 1 & 19 & 1 & $\mathrm{Q}$ \\
\hline $911+384$ & $\begin{array}{lll}09 & 14 & 36.9\end{array}$ & $\begin{array}{lll}38 & 16 & 42\end{array}$ & 1580 & 89 & 850 & 34 & 650 & 36 & 235 & 7 & 70 & 2 & 30 & 1 & \\
\hline $11+395$ & 091438.5 & 392240 & 910 & 61 & 500 & 21 & 390 & 32 & 134 & 4 & 36 & 1 & 16 & 1 & \\
\hline $11+418$ & 091445.6 & 413708 & 2160 & 115 & 1220 & 49 & 1000 & 42 & 454 & 14 & 192 & 4 & 114 & 2 & $\mathrm{~g}$ \\
\hline $12+388$ & $\begin{array}{llll}09 & 15 & 34.3\end{array}$ & 383756 & 770 & 56 & 470 & 19 & 370 & 32 & 133 & 4 & 41 & 1 & 15 & 1 & \\
\hline $12+392$ & \begin{tabular}{|llll}
09 & 16 & 05.1
\end{tabular} & $\begin{array}{lll}39 & 00 & 19\end{array}$ & 560 & 49 & 250 & 11 & 210 & 31 & 58 & 2 & 19 & 1 & 7 & 1 & $\mathrm{Q}$ \\
\hline $913+385$ & $\begin{array}{llll}09 & 16 & 47.9\end{array}$ & $\begin{array}{llll}38 & 18 & 07\end{array}$ & 1500 & 85 & 920 & 37 & 700 & 37 & 324 & 10 & 139 & 3 & 63 & 2 & $\mathrm{~g}$ \\
\hline $0913+391$ & $\begin{array}{llll}09 & 16 & 48.9\end{array}$ & 385428 & 2270 & 120 & 1660 & 67 & 1650 & 58 & 1042 & 31 & 712 & 14 & 487 & 10 & $\mathrm{Q}$ \\
\hline $14+390$ & $\begin{array}{llll}09 & 17 & 41.8\end{array}$ & $3849 \quad 17$ & 830 & 58 & 400 & 17 & 330 & 32 & 87 & 3 & 21 & 1 & 9 & 1 & \\
\hline $17+458 \mathrm{~A}$ & $\begin{array}{llll}09 & 21 & 08.4\end{array}$ & 453901 & 55650 & 2783 & 29940 & 1198 & 24300 & 730 & 8124 & 244 & 2571 & 257 & 1279 & 26 & $\mathrm{~g}$ \\
\hline $918+381$ & $\begin{array}{llll}09 & 21 & 46.1\end{array}$ & 375408 & 5370 & 271 & 3120 & 125 & 2450 & 79 & 835 & 25 & 233 & 5 & 114 & 2 & $\mathrm{Q}$ \\
\hline $18+395$ & 092133.6 & $\begin{array}{llll}39 & 17 & 43\end{array}$ & 240 & 42 & 140 & 7 & 120 & 30 & 42 & 1 & 14 & 1 & 11 & 1 & $\mathrm{~g}$ \\
\hline $18+444$ & 092131.5 & 441346 & 2420 & 127 & 1430 & 57 & 1030 & 43 & 466 & 14 & 159 & 19 & 85 & 2 & $\mathrm{~g}$ \\
\hline $19+381$ & $\begin{array}{lll}09 & 22 & 15.2\end{array}$ & 375402 & 2720 & 142 & 1340 & 54 & 1050 & 44 & 292 & 9 & 80 & 2 & 28 & 1 & $\mathrm{G}$ \\
\hline $20+390$ & $\begin{array}{llll}09 & 23 & 14.4\end{array}$ & 384940 & 330 & 43 & 680 & 28 & 430 & 33 & 377 & 11 & 254 & 5 & 299 & 6 & \\
\hline $920+408$ & 092402.6 & 403460 & 2280 & 121 & 1090 & 44 & 860 & 40 & 328 & 10 & 108 & 2 & 54 & 1 & $\mathrm{~g}$ \\
\hline $921+400$ & 092449.9 & 395030 & 970 & 63 & 570 & 23 & 470 & 33 & 161 & 5 & 49 & 1 & 21 & 1 & \\
\hline $22+397$ & 092552.7 & 393506 & 770 & 56 & 340 & 14 & 240 & 31 & 56 & 2 & 8 & 1 & 2 & 1 & \\
\hline $22+407$ & 092600.4 & 402949 & 860 & 59 & 540 & 22 & 480 & 33 & 288 & 9 & 282 & 6 & 327 & 7 & $\mathrm{Q}$ \\
\hline $922+422$ & 092559.5 & 420335 & 2700 & 141 & 1270 & 51 & 970 & 42 & 265 & 8 & 64 & 2 & 21 & 1 & $\mathrm{Q}$ \\
\hline $22+425$ & 092524.1 & $42 \quad 1728$ & 3570 & 183 & 1470 & 59 & 1150 & 46 & 319 & 10 & 88 & 2 & 30 & 1 & Q \\
\hline $23+392$ & 092703.0 & $\begin{array}{lll}39 & 02 & 21\end{array}$ & 6610 & 333 & 3920 & 157 & 3330 & 104 & 2894 & 87 & 10205 & 204 & 12041 & 241 & $\mathrm{Q}$ \\
\hline $923+398$ & $\begin{array}{llll}09 & 26 & 46.8\end{array}$ & 393745 & 680 & 52 & 440 & 18 & 350 & 32 & 170 & 5 & 107 & 2 & 76 & 2 & $\mathrm{~g}$ \\
\hline $0926+388$ & 092941.2 & $\begin{array}{llll}38 & 36 & 02\end{array}$ & 1120 & 69 & 640 & 26 & 500 & 34 & 143 & 4 & 31 & 1 & 11 & 1 & $\mathrm{Q}$ \\
\hline $926+392$ & 092915.6 & 385914 & 460 & 46 & 310 & 13 & 270 & 31 & 100 & 3 & 30 & 1 & 13 & 1 & \\
\hline $29+395$ & $\begin{array}{llll}09 & 32 & 25.2\end{array}$ & 391835 & 480 & 47 & 280 & 12 & 240 & 31 & 96 & 3 & 32 & 4 & 19 & 1 & \\
\hline $30+389$ & 093306.9 & 384151 & 2210 & 118 & 1160 & 47 & 920 & 41 & 286 & 9 & 73 & 2 & 26 & 1 & G \\
\hline $30+395$ & 093341.8 & 392149 & 730 & 54 & 400 & 17 & 340 & 32 & 147 & 4 & 61 & 6 & 27 & 1 & \\
\hline $31+399$ & $\begin{array}{lll}09 & 35 & 06.7\end{array}$ & 394206 & 7330 & 369 & 3940 & 158 & 3160 & 99 & 1033 & 31 & 406 & 8 & 191 & 4 & $\mathrm{~g}$ \\
\hline $32+397$ & 093512.0 & 393301 & 880 & 59 & 410 & 17 & 280 & 31 & 117 & 4 & 31 & 5 & 12 & 1 & \\
\hline $34+387$ & 093753.3 & 383242 & 1630 & 91 & 840 & 34 & 670 & 36 & 218 & 7 & 63 & 2 & 32 & 1 & \\
\hline $0935+397$ & 093839.1 & 393420 & 460 & 46 & 360 & 15 & 320 & 31 & 154 & 5 & 83 & 8 & 45 & 1 & $\mathrm{Q}$ \\
\hline $0935+428 \mathrm{~A}$ & $0938 \quad 16.5$ & 423835 & 3150 & 163 & 1660 & 67 & 1350 & 50 & 442 & 13 & 119 & 3 & 42 & 1 & $\mathrm{G}$ \\
\hline $36+398$ & 093924.0 & 393944 & 720 & 54 & 390 & 16 & 330 & 32 & 107 & 3 & 28 & 4 & 12 & 1 & \\
\hline $36+405$ & $\begin{array}{lll}09 & 39 & 20.5\end{array}$ & 401651 & 5650 & 285 & 2770 & 111 & 2120 & 70 & 598 & 18 & 139 & 3 & 42 & 1 & $\mathrm{~g}$ \\
\hline $37+391$ & 094103.7 & 385354 & 4530 & 230 & 2460 & 99 & 1880 & 64 & 669 & 20 & 214 & 4 & 104 & 2 & $\mathrm{Q}$ \\
\hline $37+396$ & $0940 \quad 44.3$ & 392835 & & & 160 & 8 & 150 & 30 & 42 & 1 & 15 & 1 & 6 & 1 & \\
\hline $38+399 \mathrm{~B}$ & 094124.1 & 394443 & 10340 & 519 & 7240 & 291 & 4860 & 149 & 2035 & 61 & 828 & 83 & 342 & 7 & $\mathrm{~g}$ \\
\hline $942+394$ & 094556.2 & 391457 & 260 & 42 & 140 & 7 & 100 & 30 & 48 & 2 & 17 & 1 & 8 & 1 & \\
\hline $942+399$ & $\begin{array}{lll}09 & 46 & 01.4\end{array}$ & 394251 & & & 130 & 7 & 190 & 31 & 73 & 2 & 30 & 1 & 15 & 1 & \\
\hline $0943+399$ & 094618.7 & 394417 & 2310 & 122 & 1170 & 47 & 950 & 41 & 288 & 9 & 77 & 2 & 22 & 1 & \\
\hline $0944+390 \mathrm{~A}$ & $0947 \quad 10.9$ & 385115 & & & 290 & 12 & 240 & 31 & 79 & 2 & 26 & 4 & 8 & 1 & \\
\hline $944+390 \mathrm{~B}$ & 094727.1 & 384651 & 620 & 51 & 340 & 14 & 270 & 31 & 97 & 4 & 35 & 4 & 15 & 1 & $\mathrm{~g}$ \\
\hline $44+397$ & 094749.1 & 393311 & 660 & 52 & 350 & 15 & 250 & 31 & 72 & 2 & 18 & 1 & 5 & 1 & \\
\hline $45+408$ & 094855.4 & 403944 & 3240 & 167 & 2700 & 108 & 2450 & 79 & 1606 & 48 & 1910 & 38 & 1392 & 28 & $\mathrm{Q}$ \\
\hline $45+419$ & 094830.2 & 414143 & 5420 & 274 & 2960 & 118 & 2210 & 73 & 613 & 18 & 138 & 3 & 41 & 1 & \\
\hline $47+405$ & 095028.2 & 401756 & 1030 & 65 & 530 & 22 & 440 & 33 & 127 & 4 & 35 & 1 & 16 & 1 & \\
\hline $947+424$ & 095018.5 & 421258 & 3780 & 193 & 2020 & 81 & 1480 & 54 & 532 & 16 & 147 & 3 & 60 & 2 & \\
\hline $48+390$ & 095201.3 & 385032 & 800 & 57 & 500 & 21 & 400 & 32 & 144 & 4 & 46 & 1 & 15 & 1 & $\mathrm{~g}$ \\
\hline $950+402$ & 095402.6 & 395951 & 1460 & 83 & 680 & 28 & 550 & 34 & 146 & 4 & 37 & 1 & 13 & 1 & \\
\hline $951+398$ & 095430.3 & 393759 & 590 & 50 & 310 & 13 & 270 & 31 & 97 & 3 & 34 & 5 & 15 & 1 & $\mathrm{~g}$ \\
\hline $51+408$ & 095443.2 & 403644 & 2180 & 116 & 1210 & 49 & 920 & 41 & 321 & 10 & 93 & 2 & 34 & 1 & $\mathrm{Q}$ \\
\hline $0951+422$ & 095412.6 & 420108 & 2210 & 118 & 1310 & 53 & 1080 & 44 & 429 & 13 & 159 & 3 & 110 & 2 & $\mathrm{~g}$ \\
\hline $0953+382$ & 095644.3 & $\begin{array}{lll}38 & 03 & 02\end{array}$ & 1390 & 80 & 770 & 31 & 650 & 36 & 216 & 7 & 67 & 2 & 29 & 1 & \\
\hline $53+398$ & 095608.5 & 393515 & 860 & 59 & 590 & 24 & 500 & 34 & 295 & 9 & 247 & 5 & 129 & 3 & $\mathrm{Q}$ \\
\hline $54+436$ & 095745.6 & 432649 & 2460 & 129 & 1420 & 60 & 1110 & 45 & 419 & 13 & 142 & 3 & 51 & 1 & $\mathrm{~g}$ \\
\hline $0955+380$ & 095830.7 & $3747 \quad 16$ & 1090 & 68 & 590 & 24 & 460 & 33 & 138 & 4 & 28 & 1 & 11 & 1 & \\
\hline $0955+387$ & $\begin{array}{llll}09 & 58 & 02.7\end{array}$ & 382959 & 3450 & 177 & 1820 & 73 & 1420 & 52 & 448 & 13 & 116 & 3 & 46 & 1 & $\mathrm{Q}$ \\
\hline $0955+390$ & 095844.2 & 384822 & 1910 & 104 & 1290 & 52 & 1050 & 44 & 462 & 14 & 156 & 3 & 59 & 2 & \\
\hline $0955+396$ & 095856.8 & 392230 & 1130 & 69 & 700 & 28 & 490 & 33 & 168 & 5 & 41 & 1 & 18 & 1 & \\
\hline $0956+391$ & 095943.6 & $\begin{array}{llll}38 & 57 & 02\end{array}$ & 690 & 53 & 390 & 26 & 280 & 31 & 98 & 3 & 29 & 4 & 12 & 1 & \\
\hline $0956+404$ & 095937.4 & 401528 & 1160 & 70 & 590 & 24 & 470 & 33 & 125 & 4 & 25 & 1 & 7 & 1 & \\
\hline $0956+475$ & 095918.8 & 472114 & 6130 & 309 & 2820 & 113 & 2100 & 70 & 509 & 15 & 94 & 2 & 30 & 1 & $\mathrm{G}$ \\
\hline $0957+399$ & $\begin{array}{llll}10 & 00 & 46.1\end{array}$ & 394046 & 620 & 51 & 300 & 13 & 240 & 31 & 69 & 2 & 14 & 1 & 4 & 1 & \\
\hline $0958+390 \mathrm{~A}$ & $\begin{array}{llll}10 & 01 & 24.5\end{array}$ & 384756 & & & 440 & 18 & 340 & 32 & 116 & 4 & 32 & 4 & 16 & 1 & \\
\hline $0958+391$ & $\begin{array}{llll}10 & 01 & 18.5\end{array}$ & 385147 & & & 280 & 12 & 220 & 31 & 68 & 3 & 9 & 1 & 3 & 1 & \\
\hline $1004+446$ & $\begin{array}{llll}10 & 07 & 19.5\end{array}$ & 442513 & 8690 & 436 & 5000 & 200 & 4220 & 130 & 1384 & 42 & 411 & 8 & 168 & 4 & \\
\hline $1007+417$ & $\begin{array}{lll}10 & 10 & 27.5\end{array}$ & 413229 & 8450 & 424 & 4820 & 193 & 4100 & 127 & 1688 & 51 & 649 & 13 & 335 & 7 & $\mathrm{Q}$ \\
\hline $1007+422$ & $\begin{array}{lll}10 & 10 & 24.7\end{array}$ & 415932 & 1580 & 89 & 1120 & 45 & 1000 & 42 & 436 & 13 & 146 & 3 & 73 & 2 & \\
\hline $1008+395$ & 101155.2 & 392004 & 410 & 45 & 180 & 9 & 170 & 30 & 67 & 2 & 24 & 1 & 8 & 1 & \\
\hline $1008+423$ & 101154.1 & 420433 & 1050 & 66 & 1070 & 43 & 1120 & 45 & 596 & 18 & 237 & 5 & 101 & 2 & \\
\hline
\end{tabular}


Table 2. continued

\begin{tabular}{|c|c|c|c|c|c|c|c|c|c|c|c|c|c|c|c|}
\hline B3 name & $\begin{array}{l}\mathrm{RA}(\mathrm{J} 2000) \\
{\left[\begin{array}{ccc}\mathrm{h} & \mathrm{m} & \mathrm{s}\end{array}\right]}\end{array}$ & 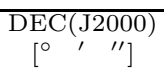 & $\begin{array}{r}S_{151} \\
{[\mathrm{mJy}]}\end{array}$ & $\begin{array}{r}\Delta S_{151} \\
{[\mathrm{mJy}]}\end{array}$ & $\begin{array}{r}S_{327} \\
{[\mathrm{mJy}]}\end{array}$ & $\begin{array}{r}\Delta S_{327} \\
{[\mathrm{mJy}]}\end{array}$ & $\begin{array}{r}S_{408} \\
{[\mathrm{mJy}]}\end{array}$ & $\begin{array}{r}\Delta S_{408} \\
{[\mathrm{mJy}]}\end{array}$ & $\begin{array}{l}S_{1400} \\
{[\mathrm{mJy}]}\end{array}$ & $\begin{array}{r}\Delta S_{1400} \\
{[\mathrm{mJy}]}\end{array}$ & $\begin{array}{l}S_{4850} \\
{[\mathrm{mJy}]}\end{array}$ & $\begin{array}{r}\Delta S_{4850} \\
{[\mathrm{mJy}]}\end{array}$ & $\begin{array}{r}S_{10550} \\
{[\mathrm{mJy}]}\end{array}$ & $\begin{array}{r}\Delta S_{10550} \\
{[\mathrm{mJy}]}\end{array}$ & Id. \\
\hline $1008+467 \mathrm{~A}$ & \begin{tabular}{|llll}
10 & 11 & 45.6
\end{tabular} & 462820 & 18030 & 902 & 8530 & 341 & 6550 & 199 & 1556 & 47 & 321 & 7 & 93 & 2 & $\bar{G}$ \\
\hline $1009+389 \mathrm{~A}$ & $\begin{array}{lll}10 & 12 & 24.4\end{array}$ & 384405 & 640 & 51 & 380 & 16 & 290 & 31 & 114 & 3 & 32 & 5 & 17 & 1 & \\
\hline $1009+434$ & $\begin{array}{lll}10 & 12 & 09.7\end{array}$ & $43 \quad 1305$ & 2890 & 150 & 1280 & 56 & 910 & 41 & 195 & 6 & 37 & 1 & 9 & 1 & G \\
\hline $1012+389$ & 101556.8 & 384152 & 700 & 53 & 390 & 16 & 330 & 32 & 102 & 3 & 35 & 5 & 19 & 1 & \\
\hline $1012+395$ & $\begin{array}{lll}10 & 15 & 50.8\end{array}$ & 392007 & 360 & 44 & 220 & 10 & 180 & 30 & 55 & 2 & 14 & 1 & 9 & 1 & \\
\hline $1012+425$ & $1015 \quad 32.5$ & $42 \quad 1948$ & 2390 & 126 & 1200 & 48 & 900 & 40 & 273 & 8 & 70 & 2 & 24 & 1 & \\
\hline $1013+410$ & $\begin{array}{lll}10 & 15 & 57.7\end{array}$ & 404648 & 6580 & 331 & 3480 & 139 & 2860 & 91 & 1075 & 32 & 344 & 36 & 186 & 4 & $\mathrm{~g}$ \\
\hline $1014+392$ & $\begin{array}{llll}10 & 17 & 14.1\end{array}$ & $\begin{array}{lll}39 & 01 & 23\end{array}$ & 5550 & 280 & 3640 & 146 & 3080 & 97 & 1398 & 42 & 517 & 10 & 229 & 5 & g \\
\hline $1014+397 \mathrm{~A}$ & $\begin{array}{llll}10 & 17 & 17.6\end{array}$ & 393132 & 5560 & 281 & 2860 & 114 & 2150 & 71 & 692 & 21 & 196 & 22 & 110 & 2 & $\mathrm{~g}$ \\
\hline $1015+383$ & $\begin{array}{lll}10 & 18 & 25.6\end{array}$ & $\begin{array}{lll}38 & 05 & 28\end{array}$ & 1270 & 75 & 720 & 29 & 640 & 36 & 271 & 8 & 108 & 2 & 66 & 2 & $\mathrm{Q}$ \\
\hline $1016+388 \mathrm{~B}$ & $\begin{array}{lll}10 & 19 & 47.2\end{array}$ & 383335 & 1120 & 69 & 640 & 26 & 430 & 33 & 149 & 4 & 73 & 12 & 19 & 1 & \\
\hline $1016+396$ & $\begin{array}{llll}10 & 19 & 27.0\end{array}$ & 392241 & 400 & 45 & 190 & 9 & 140 & 30 & 23 & 1 & 3 & 1 & $<3$ & & \\
\hline $1016+397$ & $\begin{array}{lll}10 & 1955.0\end{array}$ & 393020 & 200 & 41 & 150 & 8 & 110 & 30 & 57 & 2 & 19 & 4 & 9 & 1 & $\mathrm{~g}$ \\
\hline $1016+443$ & $\begin{array}{lll}10 & 19 & 48.2\end{array}$ & 440825 & 1050 & 66 & 1260 & 51 & 1000 & 42 & 359 & 11 & 71 & 2 & 21 & 1 & $\mathrm{~g}$ \\
\hline $1018+393$ & 102137.8 & 390355 & 280 & 42 & 240 & 11 & 240 & 31 & 75 & 2 & 24 & 1 & 10 & 1 & \\
\hline $1018+405$ & $\begin{array}{lll}10 & 21 & 44.9\end{array}$ & $40 \quad 1939$ & 1620 & 90 & 890 & 36 & 670 & 36 & 213 & 6 & 57 & 2 & 21 & 1 & \\
\hline $1019+382 \mathrm{~A}$ & $\begin{array}{lll}10 & 22 & 23.0\end{array}$ & 380252 & 1130 & 69 & 630 & 26 & 560 & 34 & 212 & 6 & 79 & 2 & 40 & 1 & \\
\hline $1019+394$ & 102255.2 & $3908 \quad 49$ & 2990 & 155 & 1720 & 69 & 1400 & 52 & 480 & 14 & 141 & 3 & 45 & 1 & $\mathrm{G}$ \\
\hline $1019+395$ & $1022 \quad 15.6$ & $\begin{array}{lll}39 & 17 & 29\end{array}$ & 510 & 47 & 360 & 15 & 280 & 31 & 94 & 4 & 31 & 4 & 8 & 1 & \\
\hline $1019+397$ & $\begin{array}{lll}10 & 22 & 38.2\end{array}$ & 393151 & 240 & 42 & 200 & 9 & 170 & 30 & 96 & 3 & 48 & 6 & 27 & 1 & $\mathrm{Q}$ \\
\hline $1020+400$ & 102311.6 & $3948 \quad 17$ & 3020 & 156 & 2060 & 83 & 1770 & 61 & 1129 & 34 & 689 & 14 & 852 & 17 & $\mathrm{Q}$ \\
\hline $1021+384$ & 102356.4 & $\begin{array}{lll}38 & 08 & 47\end{array}$ & 990 & 64 & 680 & 28 & 570 & 35 & 250 & 8 & 82 & 2 & 41 & 1 & \\
\hline $1022+432$ & $\begin{array}{lll}10 & 25 & 29.8\end{array}$ & 425743 & 6400 & 322 & 3430 & 137 & 2680 & 86 & 858 & 26 & 224 & 5 & 76 & 2 & \\
\hline $1023+393$ & $\begin{array}{lll}10 & 26 & 04.4\end{array}$ & 390523 & 700 & 53 & 390 & 16 & 320 & 31 & 131 & 4 & 54 & 6 & 16 & 1 & $\mathrm{~g}$ \\
\hline $1024+463$ & $\begin{array}{lll}10 & 27 & 14.9\end{array}$ & 460251 & 8690 & 436 & 5170 & 208 & 4090 & 126 & 1408 & 42 & 386 & 8 & 215 & 4 & $\mathrm{G}$ \\
\hline $1025+390 \mathrm{~B}$ & 102844.3 & 384436 & 2970 & 154 & 1830 & 73 & 1540 & 55 & 669 & 20 & 301 & 6 & 220 & 5 & $\mathrm{~g}$ \\
\hline $1025+394$ & 102851.2 & $\begin{array}{lll}39 & 10 & 47\end{array}$ & 760 & 55 & 350 & 15 & 290 & 31 & 82 & 3 & 24 & 4 & 13 & 1 & \\
\hline $1027+383$ & $\begin{array}{lll}10 & 30 & 40.3\end{array}$ & $\begin{array}{lll}38 & 0322\end{array}$ & 1830 & 100 & 970 & 39 & 720 & 37 & 287 & 9 & 83 & 2 & 32 & 1 & \\
\hline $1027+390$ & $\begin{array}{llll}10 & 30 & 26.3\end{array}$ & $\begin{array}{llll}38 & 47 & 01\end{array}$ & 530 & 48 & 280 & 12 & 230 & 31 & 69 & 2 & 24 & 1 & 12 & 1 & \\
\hline $1027+392$ & $\begin{array}{lll}10 & 30 & 16.5\end{array}$ & 385754 & 1260 & 75 & 940 & 38 & 830 & 39 & 389 & 12 & 150 & 3 & 81 & 2 & \\
\hline $1028+400$ & $\begin{array}{llll}10 & 31 & 17.0\end{array}$ & 394656 & 780 & 56 & 500 & 21 & 420 & 33 & 159 & 5 & 50 & 1 & 20 & 1 & \\
\hline $1028+402$ & $\begin{array}{llll}10 & 31 & 02.8\end{array}$ & $40 \quad 0044$ & 2050 & 110 & 1030 & 41 & 820 & 39 & 250 & 8 & 66 & 2 & 26 & 1 & \\
\hline $1030+398$ & 103322.1 & 393551 & 640 & 51 & 830 & 34 & 700 & 37 & 410 & 12 & 711 & 14 & 382 & 8 & $\mathrm{G}$ \\
\hline $1030+415$ & $\begin{array}{lll}10 & 33 & 03.7\end{array}$ & 411606 & 1320 & 77 & 770 & 31 & 1020 & 43 & 491 & 15 & 273 & 6 & 230 & 5 & $\mathrm{Q}$ \\
\hline $1033+387 \mathrm{~A}$ & $\begin{array}{llll}10 & 36 & 23.4\end{array}$ & 383133 & & & 600 & 24 & & & 132 & 6 & 31 & 10 & 22 & 1 & $\mathrm{~g}$ \\
\hline $1033+387 \mathrm{~B}$ & 103645.8 & 382645 & 700 & 53 & 570 & 23 & 360 & 32 & 120 & 4 & 22 & 1 & 8 & 1 & \\
\hline $1033+388$ & 103634.9 & 383533 & & & 510 & 45 & & & 184 & 7 & 63 & 12 & 32 & 1 & $\mathrm{~g}$ \\
\hline $1033+408$ & 103625.9 & 403527 & 2270 & 120 & 1180 & 47 & 950 & 41 & 295 & 9 & 78 & 2 & 27 & 1 & \\
\hline $1034+397$ & 103734.0 & 392800 & & & 90 & 6 & 100 & 30 & 27 & 1 & 12 & 1 & 7 & 1 & \\
\hline $1034+404$ & $\begin{array}{llll}10 & 37 & 12.4\end{array}$ & 401138 & 4260 & 217 & 2560 & 103 & 2150 & 71 & 769 & 23 & 231 & 5 & 84 & 2 & $\mathrm{G}$ \\
\hline $1035+398$ & $1038 \quad 16.9$ & 393255 & 460 & 46 & 260 & 11 & 210 & 31 & 90 & 3 & 33 & 5 & 17 & 1 & \\
\hline $1037+399$ & $\begin{array}{lll}10 & 40 & 12.3\end{array}$ & $3942 \quad 26$ & 870 & 59 & 390 & 16 & 280 & 31 & 84 & 4 & 24 & 1 & 9 & 1 & \\
\hline $1038+398$ & $\begin{array}{lll}10 & 41 & 29.2\end{array}$ & 393356 & 890 & 60 & 460 & 19 & 360 & 32 & 124 & 4 & 40 & 1 & 16 & 1 & \\
\hline $1039+397$ & $\begin{array}{lll}10 & 42 & 01.2\end{array}$ & 392659 & 1370 & 79 & 830 & 34 & 660 & 36 & 294 & 9 & 116 & 3 & 49 & 1 & $\mathrm{~g}$ \\
\hline $1039+424$ & $\begin{array}{lll}10 & 42 & 06.2\end{array}$ & $42 \quad 1030$ & 1770 & 97 & 1060 & 43 & 870 & 40 & 281 & 8 & 66 & 2 & 23 & 1 & \\
\hline $1040+395$ & 104305.3 & $3914 \quad 16$ & 1690 & 93 & 970 & 39 & 760 & 38 & 284 & 9 & 83 & 13 & 50 & 1 & \\
\hline $1040+397$ & $1043 \quad 17.5$ & 392938 & 370 & 44 & 190 & 9 & 130 & 30 & 46 & 1 & 17 & 1 & 8 & 1 & \\
\hline $1040+398$ & 104336.0 & 393412 & & & 90 & 6 & 110 & 30 & 46 & 2 & 23 & 1 & 9 & 1 & $\mathrm{~g}$ \\
\hline $1041+392$ & 104426.2 & 390050 & 1170 & 71 & 740 & 30 & 600 & 35 & 226 & 7 & 76 & 2 & 30 & 1 & \\
\hline $1042+392$ & $1045 \quad 15.0$ & 385638 & 2680 & 140 & 1920 & 77 & 1500 & 54 & 643 & 19 & 236 & 5 & 88 & 2 & \\
\hline $1042+393$ & $\begin{array}{lll}10 & 45 & 21.2\end{array}$ & $\begin{array}{lll}39 & 04 & 19\end{array}$ & & & 270 & 12 & 280 & 31 & 74 & 2 & 20 & 1 & 8 & 1 & \\
\hline $1042+397$ & 104540.3 & 393008 & 270 & 42 & 220 & 10 & 170 & 30 & 108 & 3 & 46 & 5 & 27 & 1 & \\
\hline $1043+394$ & 104557.9 & $\begin{array}{lll}39 & 1307\end{array}$ & 660 & 52 & 350 & 15 & 300 & 31 & 110 & 3 & 26 & 4 & 14 & 1 & \\
\hline $1044+454$ & 104733.7 & 450853 & 3120 & 161 & 1730 & 69 & 1670 & 58 & 444 & 13 & 87 & 2 & 24 & 1 & \\
\hline $1047+387$ & 105046.0 & 383148 & 460 & 46 & 420 & 17 & 420 & 33 & 198 & 6 & 69 & 2 & 28 & 1 & \\
\hline $1047+396$ & $\begin{array}{lll}10 & 50 & 13.7\end{array}$ & 392543 & 450 & 46 & 220 & 10 & 160 & 30 & 57 & 3 & 16 & 1 & 6 & 1 & \\
\hline $1049+384$ & $\begin{array}{lll}10 & 52 & 11.8\end{array}$ & $\begin{array}{llll}38 & 11 & 42\end{array}$ & 840 & 58 & 1380 & 55 & 1240 & 48 & 693 & 21 & 203 & 4 & 71 & 2 & \\
\hline $1050+391$ & 105333.7 & 385348 & 860 & 59 & 480 & 20 & 370 & 32 & 146 & 4 & 45 & 1 & 19 & 1 & \\
\hline $1052+380$ & 105544.5 & 374601 & 1790 & 98 & 1080 & 43 & 820 & 39 & 222 & 7 & 40 & 1 & 11 & 1 & \\
\hline $1052+389$ & 105525.0 & 384018 & 640 & 51 & 300 & 13 & 240 & 31 & 68 & 2 & 17 & 1 & 5 & 1 & \\
\hline $1053+384$ & $1056 \quad 12.4$ & $\begin{array}{llll}38 & 08 & 43\end{array}$ & 1510 & 85 & 910 & 37 & 750 & 37 & 298 & 9 & 105 & 2 & 52 & 1 & \\
\hline $1053+394$ & 105648.8 & 391125 & 650 & 52 & 350 & 15 & 280 & 31 & 111 & 3 & 37 & 5 & 16 & 1 & \\
\hline $1054+396$ & 105726.8 & $\begin{array}{lll}39 & 25 & 15\end{array}$ & 270 & 42 & 210 & 10 & 180 & 30 & 77 & 2 & 27 & 4 & 17 & 1 & \\
\hline $1055+381$ & $1058 \quad 08.3$ & 375415 & 1170 & 71 & 770 & 31 & 600 & 35 & 253 & 8 & 93 & 2 & 47 & 1 & $G$ \\
\hline $1055+396$ & $1058 \quad 40.3$ & $39 \quad 1946$ & 420 & 45 & 330 & 14 & & & 120 & 6 & 16 & 3 & 20 & 1 & \\
\hline $1055+404 \mathrm{~A}$ & 105800.3 & $40 \quad 1019$ & 1910 & 104 & 1140 & 46 & 940 & 41 & 371 & 11 & 124 & 3 & 49 & 1 & \\
\hline $1055+404 \mathrm{~B}$ & 105837.9 & 401407 & 2360 & 125 & 1320 & 53 & 1080 & 44 & 370 & 11 & 117 & 3 & 49 & 1 & \\
\hline $1056+387$ & $\begin{array}{llll}10 & 59 & 16.3\end{array}$ & 382554 & 1930 & 104 & 1110 & 45 & 860 & 40 & 277 & 8 & 81 & 2 & 26 & 1 & \\
\hline $1056+396$ & $\begin{array}{llll}10 & 59 & 11.3\end{array}$ & 392505 & 1700 & 94 & 1070 & 43 & 820 & 39 & 288 & 9 & 76 & 2 & 22 & 1 & $\mathrm{G}$ \\
\hline $1056+399$ & $\begin{array}{lll}10 & 59 & 03.7\end{array}$ & 394402 & 210 & 41 & 170 & 8 & 140 & 30 & 87 & 3 & 45 & 1 & 24 & 1 & \\
\hline $1056+432 \mathrm{~A}$ & 105858.8 & 430124 & 12960 & 649 & 8400 & 336 & 7010 & 212 & 2889 & 87 & 906 & 18 & 329 & 7 & $G$ \\
\hline $1058+393$ & $\begin{array}{lll}11 & 0130.0\end{array}$ & 390432 & & & 260 & 11 & 340 & 32 & 338 & 10 & 266 & 24 & 177 & 4 & \\
\hline $1100+398$ & $\begin{array}{lll}11 & 03 & 23.8\end{array}$ & 393346 & 300 & 43 & 190 & 9 & 140 & 30 & 48 & 2 & 14 & 1 & 6 & 1 & \\
\hline $1101+384$ & $\begin{array}{llll}11 & 04 & 27.2\end{array}$ & $\begin{array}{llll}38 & 12 & 32\end{array}$ & 1680 & 93 & 1370 & 55 & 1140 & 45 & 891 & 27 & 676 & 14 & 522 & 10 & $\mathrm{~g}$ \\
\hline $1101+395$ & $\begin{array}{llll}11 & 04 & 37.7\end{array}$ & 391451 & 470 & 46 & 250 & 11 & 190 & 31 & 54 & 2 & 10 & 1 & 5 & 1 & \\
\hline $1101+396$ & $\begin{array}{lll}11 & 04 & 02.8\end{array}$ & 392055 & 350 & 44 & 170 & 8 & 130 & 30 & 27 & 1 & 3 & 1 & $<5$ & & \\
\hline $1103+393$ & $\begin{array}{lll}11 & 06 & 08.2\end{array}$ & 390604 & 1170 & 71 & 660 & 27 & 520 & 34 & 190 & 6 & 62 & 2 & 28 & 1 & \\
\hline $1104+390$ & $\begin{array}{lll}11 & 07 & 40.3\end{array}$ & $\begin{array}{lll}38 & 49 & 03\end{array}$ & 370 & 44 & 230 & 10 & 200 & 31 & 71 & 2 & 25 & 1 & 15 & 1 & \\
\hline $1104+397$ & $\begin{array}{llll}11 & 07 & 01.0\end{array}$ & $\begin{array}{lll}39 & 29 & 13\end{array}$ & 350 & 44 & 160 & 8 & 120 & 30 & 30 & 2 & 5 & 1 & $<4$ & & \\
\hline
\end{tabular}


Table 2. continued

\begin{tabular}{|c|c|c|c|c|c|c|c|c|c|c|c|c|c|c|c|}
\hline B3 name & $\begin{array}{c}\text { RA(J2000) } \\
{\left[\begin{array}{lll}\mathrm{h} & \mathrm{m} & \mathrm{s}\end{array}\right]}\end{array}$ & $\begin{array}{c}\mathrm{DEC}(\mathrm{J} 2000) \\
{\left[{ }^{\circ},{ }^{\prime}\right]}\end{array}$ & $\begin{array}{r}S_{151} \\
{[\mathrm{mJy}]}\end{array}$ & $\begin{array}{r}\Delta S_{151} \\
{[\mathrm{mJy}]}\end{array}$ & $\begin{array}{r}S_{327} \\
{[\mathrm{mJy}]}\end{array}$ & $\begin{array}{r}\Delta S_{327} \\
{[\mathrm{mJy}]}\end{array}$ & $\begin{array}{r}S_{408} \\
{[\mathrm{mJy}]}\end{array}$ & $\begin{array}{r}\Delta S_{408} \\
{[\mathrm{mJy}]}\end{array}$ & $\begin{array}{l}S_{1400} \\
{[\mathrm{mJy}]}\end{array}$ & $\begin{array}{r}\Delta S_{1400} \\
{[\mathrm{mJy}]}\end{array}$ & $\begin{array}{l}S_{4850} \\
{[\mathrm{mJy}]}\end{array}$ & $\begin{array}{r}\Delta S_{4850} \\
{[\mathrm{mJy}]}\end{array}$ & $\begin{array}{r}S_{10550} \\
{[\mathrm{mJy}]}\end{array}$ & $\begin{array}{r}\Delta S_{10550} \\
{[\mathrm{mJy}]}\end{array}$ & Id. \\
\hline $1105+390$ & $\begin{array}{llll}11 & 08 & 22.5 \\
\end{array}$ & $38 \quad 4552$ & 470 & 46 & 280 & 12 & 230 & 31 & 91 & 3 & 29 & 5 & 19 & 1 & $\mathrm{~g}$ \\
\hline $1105+392$ & $\begin{array}{llll}11 & 08 & 37.9\end{array}$ & $38 \quad 58 \quad 39$ & 4990 & 253 & 2900 & 116 & 2310 & 76 & 898 & 27 & 276 & 6 & 128 & 3 & Q \\
\hline $1106+380$ & $\begin{array}{lll}11 & 09 & 28.8\end{array}$ & 374432 & 1920 & 104 & 1370 & 55 & 1290 & 49 & 1226 & 37 & 769 & 15 & 502 & 10 & G \\
\hline $1107+379$ & $\begin{array}{lll}11 & 09 & 49.6\end{array}$ & 373829 & 10510 & 527 & 7030 & 281 & 5630 & 172 & 2045 & 61 & 666 & 13 & 293 & 6 & $\mathrm{~g}$ \\
\hline $1108+394$ & $\begin{array}{lll}11 & 11 & 14.3\end{array}$ & $39 \quad 12 \quad 33$ & 300 & 43 & 90 & 6 & 100 & 30 & 25 & 2 & 6 & 1 & $<5$ & & \\
\hline $1108+399$ & 111119.5 & $3940 \quad 14$ & 2100 & 112 & 1200 & 48 & 930 & 41 & 369 & 11 & 148 & 3 & 79 & 2 & $\mathrm{~g}$ \\
\hline $1108+411 \mathrm{~B}$ & $\begin{array}{lll}11 & 1139.0\end{array}$ & 405016 & 7880 & 396 & 3980 & 159 & 3200 & 101 & 844 & 25 & 256 & 5 & 97 & 2 & $\mathrm{~g}$ \\
\hline $1109+437$ & $\begin{array}{lll}11 & 12 & 39.2\end{array}$ & 432602 & 9810 & 492 & 6000 & 240 & 4770 & 146 & 1429 & 43 & 337 & 7 & 145 & 3 & Q \\
\hline $1110+391$ & $11 \quad 1326.6$ & 385325 & 800 & 57 & 400 & 17 & 320 & 31 & 98 & 3 & 24 & 1 & 14 & 1 & \\
\hline $1111+391$ & $\begin{array}{lll}11 & 14 & 29.3\end{array}$ & 385035 & 600 & 50 & 450 & 19 & 370 & 32 & 174 & 5 & 61 & 2 & 24 & 1 & \\
\hline $1111+396 \mathrm{~A}$ & $11 \quad 1437.9$ & $\begin{array}{lll}39 & 27 & 14\end{array}$ & & & 110 & 6 & & & 39 & 1 & 13 & 1 & 5 & 1 & \\
\hline $1111+408$ & 111438.4 & 403720 & 27610 & 1381 & 13990 & 560 & 10920 & 329 & 3125 & 94 & 752 & 15 & 249 & 5 & $\mathrm{Q}$ \\
\hline $1112+435$ & $\begin{array}{lll}11 & 15 & 20.7\end{array}$ & 431443 & 1710 & 94 & 990 & 40 & 830 & 39 & 330 & 10 & 128 & 3 & 64 & 2 & $\mathrm{~g}$ \\
\hline $1115+380 \mathrm{~A}$ & $11 \quad 1804.5$ & $\begin{array}{lll}37 & 48 & 07\end{array}$ & 1600 & 89 & 1080 & 43 & 700 & 37 & 225 & 7 & 70 & 2 & 35 & 1 & \\
\hline $1115+399$ & $\begin{array}{lll}11 & 1757.0\end{array}$ & 394044 & 350 & 44 & 170 & 8 & 150 & 30 & 61 & 2 & 30 & 4 & 19 & 1 & \\
\hline $1116+388$ & $\begin{array}{lll}11 & 1855.0\end{array}$ & 383425 & & & 270 & 12 & 200 & 31 & 97 & 3 & 35 & 5 & 13 & 1 & \\
\hline $1116+392$ & $\begin{array}{lll}11 & 19 & 03.4\end{array}$ & 385852 & 1060 & 66 & 520 & 21 & 360 & 32 & 141 & 4 & 42 & 1 & 16 & 1 & Q \\
\hline $1117+441$ & $\begin{array}{lll}11 & 20 & 16.0\end{array}$ & 435451 & 2190 & 117 & 1290 & 52 & 1010 & 43 & 396 & 12 & 134 & 3 & 63 & 2 & \\
\hline $1118+390$ & $\begin{array}{lll}11 & 21 & 12.7\end{array}$ & 384408 & 530 & 48 & 250 & 11 & 200 & 31 & 73 & 3 & 22 & 4 & 7 & 1 & \\
\hline $1121+399$ & 112347.1 & 393749 & 750 & 55 & 540 & 22 & 460 & 33 & 218 & 7 & 82 & 2 & 39 & 1 & \\
\hline $1121+435$ & 112432.1 & $43 \quad 1540$ & 2590 & 136 & 1460 & 59 & 1200 & 47 & 411 & 12 & 120 & 3 & 62 & 2 & \\
\hline $1121+444$ & 112354.9 & 440834 & 3690 & 189 & 1830 & 73 & 1390 & 51 & 383 & 12 & 69 & 2 & 31 & 1 & \\
\hline $1122+390$ & 112443.5 & 384547 & 400 & 45 & 270 & 12 & 220 & 31 & 138 & 4 & 52 & 6 & 36 & 1 & $\mathrm{~g}$ \\
\hline $1122+397$ & 112542.4 & 392531 & 630 & 51 & 380 & 16 & 290 & 31 & 126 & 4 & 44 & 1 & 22 & 1 & \\
\hline $1123+395$ & 112628.3 & $\begin{array}{llll}39 & 18 & 43\end{array}$ & 1900 & 103 & 490 & 20 & 360 & 32 & 145 & 4 & 63 & 2 & 26 & 1 & Q \\
\hline $1127+380$ & 112955.5 & 374804 & 1820 & 99 & 1080 & 43 & 780 & 38 & 247 & 7 & 75 & 2 & 31 & 1 & \\
\hline $1128+385$ & 113053.3 & $\begin{array}{lll}38 & 15 & 18\end{array}$ & 760 & 55 & 610 & 25 & 520 & 34 & 715 & 21 & 655 & 13 & 944 & 19 & Q \\
\hline $1128+392$ & $\begin{array}{lll}11 & 31 & 12.7\end{array}$ & 390034 & 1110 & 68 & 690 & 28 & 560 & 34 & 204 & 6 & 60 & 2 & 21 & 1 & \\
\hline $1128+396$ & $\begin{array}{lll}11 & 31 & 29.2\end{array}$ & 392132 & 760 & 55 & 370 & 15 & 250 & 31 & 81 & 2 & 19 & 1 & 9 & 1 & \\
\hline $1128+436$ & 113047.6 & 432504 & 3600 & 184 & 1860 & 75 & 1550 & 55 & 417 & 13 & 97 & 2 & 38 & 1 & \\
\hline $1128+455$ & 113138.9 & $45 \quad 1449$ & 6330 & 319 & 5250 & 210 & 4370 & 134 & 2016 & 60 & 649 & 13 & 231 & 5 & $\mathrm{~g}$ \\
\hline $1130+387$ & 113257.3 & 382657 & 750 & 55 & 580 & 24 & 480 & 33 & 192 & 6 & 65 & 2 & 28 & 1 & \\
\hline $1131+388$ & $\begin{array}{llll}11 & 34 & 03.8\end{array}$ & 383549 & 1530 & 86 & 930 & 37 & 750 & 37 & 313 & 9 & 124 & 3 & 63 & 2 & $\mathrm{~g}$ \\
\hline $1131+391$ & 113413.2 & $3851 \quad 17$ & 690 & 53 & 400 & 17 & 310 & 31 & 144 & 4 & 100 & 2 & 74 & 2 & \\
\hline $1131+437$ & 113438.4 & 432801 & 7210 & 363 & 4750 & 190 & 3900 & 121 & 1576 & 47 & 521 & 10 & 216 & 4 & $\mathrm{G}$ \\
\hline $1132+396$ & 113502.1 & 392302 & 330 & 43 & 170 & 8 & 110 & 30 & 35 & 1 & 6 & 1 & 4 & 1 & \\
\hline $1132+406$ & 113441.0 & 402116 & 1960 & 106 & 1050 & 42 & 770 & 38 & 271 & 8 & 88 & 2 & 41 & 1 & $\mathrm{~g}$ \\
\hline $1132+410$ & 113445.7 & 404353 & 2730 & 142 & 1420 & 57 & 1100 & 45 & 324 & 10 & 84 & 2 & 29 & 1 & \\
\hline $1133+395$ & 113626.0 & $3917 \quad 15$ & 300 & 43 & 160 & 8 & 140 & 30 & 67 & 2 & 26 & 4 & 13 & 1 & \\
\hline $1133+432$ & 113556.0 & 425844 & & & 580 & 24 & 880 & 40 & 1455 & 44 & 503 & 10 & 172 & 4 & \\
\hline $1134+406$ & 113726.8 & 402230 & 1000 & 64 & 660 & 27 & 530 & 34 & 207 & 6 & 65 & 2 & 30 & 1 & \\
\hline $1135+390$ & 113758.0 & 384508 & 620 & 51 & 280 & 12 & 220 & 31 & 74 & 2 & 21 & 1 & 8 & 1 & \\
\hline $1135+401$ & 113740.6 & 395048 & 340 & 43 & 700 & 28 & 660 & 36 & 361 & 11 & 125 & 3 & 34 & 1 & \\
\hline $1136+383$ & 113933.9 & $\begin{array}{lll}38 & 03 & 41\end{array}$ & 550 & 49 & 1010 & 41 & 840 & 39 & 462 & 14 & 174 & 4 & 84 & 2 & \\
\hline $1136+390$ & 113909.5 & $\begin{array}{llll}38 & 47 & 18\end{array}$ & 900 & 60 & 620 & 25 & 490 & 33 & 202 & 6 & 70 & 2 & 32 & 1 & \\
\hline $1136+420$ & 113859.1 & 414840 & 1900 & 103 & 1240 & 50 & 1090 & 44 & 463 & 14 & 174 & 4 & 76 & 2 & $\mathrm{G}$ \\
\hline $1137+396$ & 114025.0 & 392200 & 220 & 41 & 130 & 7 & 110 & 30 & 34 & 2 & 15 & 1 & 7 & 1 & $\mathrm{~g}$ \\
\hline $1140+394$ & $1143 \quad 14.5$ & 390911 & 870 & 59 & 440 & 18 & 320 & 31 & 98 & 3 & 25 & 1 & 8 & 1 & \\
\hline $1140+399$ & $1143 \quad 19.4$ & 393826 & 330 & 43 & 180 & 9 & 140 & 30 & 52 & 3 & 15 & 1 & 8 & 1 & \\
\hline $1141+374$ & 114426.9 & $\begin{array}{lll}37 & 08 & 40\end{array}$ & 9010 & 452 & 6020 & 241 & 4750 & 146 & 2140 & 64 & 797 & 16 & 442 & 9 & $\mathrm{~g}$ \\
\hline $1141+392$ & 114348.8 & 385842 & 1460 & 83 & 1040 & 47 & 600 & 35 & 211 & 6 & 63 & 2 & 29 & 1 & \\
\hline $1141+400$ & 114432.9 & $\begin{array}{lll}39 & 4323\end{array}$ & 860 & 59 & 510 & 21 & 410 & 32 & 157 & 5 & 59 & 2 & 27 & 1 & Q \\
\hline $1141+466$ & 114339.6 & 462120 & 5460 & 276 & 3270 & 131 & 2600 & 84 & 870 & 26 & 208 & 4 & 60 & 2 & $\mathrm{~g}$ \\
\hline $1142+392$ & 114534.0 & 385656 & 1090 & 68 & 700 & 28 & 570 & 35 & 231 & 7 & 79 & 2 & 32 & 1 & $\mathrm{Q}$ \\
\hline $1143+405$ & 114627.4 & 401458 & 1240 & 74 & 610 & 25 & 490 & 33 & 150 & 5 & 36 & 1 & 13 & 1 & \\
\hline $1143+456$ & 114615.2 & $45 \quad 2037$ & 4500 & 229 & 2940 & 118 & 2260 & 74 & 716 & 21 & 138 & 3 & 43 & 1 & G \\
\hline $1144+398$ & $\begin{array}{lll}11 & 47 & 17.2\end{array}$ & 393652 & 240 & 42 & 140 & 7 & 120 & 30 & 38 & 1 & 13 & 1 & 9 & 1 & \\
\hline $1144+402$ & 114658.3 & 395833 & 670 & 52 & 620 & 25 & 930 & 41 & 358 & 11 & 1029 & 21 & 918 & 18 & $\mathrm{Q}$ \\
\hline $1144+404$ & 114642.9 & 400801 & 960 & 62 & 610 & 25 & 510 & 34 & 188 & 6 & 60 & 2 & 35 & 1 & \\
\hline $1148+387$ & 115129.3 & 382552 & 3830 & 196 & 2310 & 93 & 1830 & 63 & 655 & 20 & 228 & 5 & 140 & 3 & $\mathrm{Q}$ \\
\hline $1148+477$ & $\begin{array}{lll}11 & 51 & 09.3\end{array}$ & 472855 & 5170 & 262 & 2890 & 116 & 2290 & 75 & 706 & 21 & 156 & 3 & 52 & 1 & Q \\
\hline $1149+390$ & 115137.8 & 384614 & 800 & 57 & 440 & 18 & 330 & 32 & 99 & 3 & 27 & 1 & 7 & 1 & \\
\hline $1149+398$ & 115144.7 & 393414 & 610 & 50 & 390 & 43 & 210 & 31 & 100 & 3 & 28 & 1 & 15 & 1 & \\
\hline $1150+388$ & 115245.1 & 383145 & 1140 & 70 & 780 & 32 & 660 & 36 & 257 & 8 & 80 & 2 & 34 & 1 & \\
\hline $1150+401$ & $\begin{array}{lll}11 & 53 & 01.2\end{array}$ & 395352 & 1240 & 74 & 710 & 29 & 550 & 34 & 164 & 5 & 38 & 1 & 17 & 1 & \\
\hline $1150+438$ & 115242.4 & $43 \quad 3619$ & 2060 & 110 & 1250 & 50 & 960 & 42 & 331 & 10 & 83 & 2 & 31 & 1 & \\
\hline $1151+383$ & 115401.3 & $\begin{array}{lll}38 & 0507\end{array}$ & & & 1760 & 71 & 1420 & 52 & 523 & 16 & 167 & 4 & 68 & 2 & $\mathrm{~g}$ \\
\hline $1151+384 \mathrm{~B}$ & 115352.8 & 381145 & 4880 & 247 & 3160 & 126 & 2470 & 80 & 1041 & 31 & 342 & 7 & 222 & 5 & $\mathrm{~g}$ \\
\hline $1151+408$ & 115354.6 & 403652 & 1280 & 75 & 1080 & 43 & 880 & 40 & 1142 & 34 & 500 & 10 & 397 & 8 & Q \\
\hline $1151+456$ & 115420.6 & $45 \quad 23 \quad 37$ & 4970 & 252 & 2990 & 122 & 2280 & 75 & 958 & 29 & 353 & 7 & 203 & 4 & $\mathrm{~g}$ \\
\hline $1153+407 \mathrm{~B}$ & 115551.6 & 403012 & 2540 & 133 & 1330 & 53 & 900 & 40 & 288 & 9 & 97 & 14 & 30 & 1 & \\
\hline $1153+451$ & 115608.9 & 445015 & 3360 & 173 & 2220 & 89 & 1790 & 62 & 731 & 22 & 227 & 5 & 106 & 2 & \\
\hline $1154+397$ & 115647.5 & $\begin{array}{lll}39 & 28 & 18\end{array}$ & 310 & 43 & 170 & 8 & 140 & 30 & 81 & 4 & 25 & 1 & 16 & 1 & $\mathrm{~g}$ \\
\hline $1154+398$ & 115729.5 & 393606 & 260 & 42 & 170 & 8 & 150 & 30 & 50 & 2 & 16 & 1 & 5 & 1 & \\
\hline $1156+389$ & $\begin{array}{lll}11 & 59 & 04.5\end{array}$ & $\begin{array}{lll}38 & 40 & 09\end{array}$ & 680 & 52 & 400 & 17 & 310 & 31 & 109 & 3 & 31 & 4 & 18 & 1 & $\mathrm{G}$ \\
\hline $1157+396$ & 115951.4 & 392418 & 500 & 47 & 200 & 9 & 160 & 30 & 50 & 2 & 10 & 1 & 3 & 1 & \\
\hline $1157+460$ & $\begin{array}{lll}12 & 00 & 31.2\end{array}$ & $45 \quad 4842$ & 2680 & 140 & 3170 & 127 & 2880 & 91 & 1165 & 35 & 320 & 6 & 121 & 3 & G \\
\hline $1158+393$ & 120055.6 & $\begin{array}{lll}39 & 02 & 57\end{array}$ & 770 & 56 & 410 & 17 & 300 & 31 & 92 & 3 & 22 & 1 & 10 & 1 & \\
\hline $1159+395$ & $\begin{array}{lll}12 & 01 & 49.9\end{array}$ & $\begin{array}{lll}39 & 19 & 10\end{array}$ & 680 & 52 & 980 & 39 & 830 & 39 & 609 & 18 & 237 & 5 & 117 & 3 & G \\
\hline
\end{tabular}


Table 2. continued

\begin{tabular}{|c|c|c|c|c|c|c|c|c|c|c|c|c|c|c|c|}
\hline B3 name & \begin{tabular}{|c}
$\mathrm{RA}(\mathrm{J} 2000)$ \\
{$\left[\begin{array}{ccc}\mathrm{h} & \mathrm{m} & \mathrm{s}\end{array}\right]$}
\end{tabular} & $\begin{array}{c}\mathrm{DEC}(\mathrm{J} 2000) \\
{\left[\begin{array}{lll} & 1 & \end{array}\right]}\end{array}$ & $\begin{array}{r}S_{151} \\
{[\mathrm{mJy}]}\end{array}$ & $\begin{array}{r}S_{151} \\
{[\mathrm{mJy}]} \\
\end{array}$ & $\begin{array}{r}S_{327} \\
{[\mathrm{mJy}]} \\
\end{array}$ & $\begin{array}{r}S_{327} \\
{[\mathrm{mJy}]} \\
\end{array}$ & $\begin{array}{r}S_{408} \\
{[\mathrm{mJy}]}\end{array}$ & $\begin{array}{l}S_{408} \\
{[\mathrm{mJy}]}\end{array}$ & $\begin{array}{l}S_{1400} \\
{[\mathrm{mJy}]}\end{array}$ & $\begin{array}{r}\Delta S_{1400} \\
{[\mathrm{mJy}]} \\
\end{array}$ & $\begin{array}{l}S_{4850} \\
{[\mathrm{mJy}]}\end{array}$ & $\begin{array}{r}S_{4850} \\
{[\mathrm{mJy}]} \\
\end{array}$ & $\begin{array}{r}S_{10550} \\
{[\mathrm{mJy}]}\end{array}$ & $\begin{array}{r}S_{10550} \\
{[\mathrm{mJy}]}\end{array}$ & Id. \\
\hline $1200+393$ & 120300.2 & 390546 & 560 & 49 & 320 & 14 & 250 & 31 & 80 & 2 & 24 & 1 & 8 & 1 & \\
\hline $1201+394$ & $\begin{array}{llll}12 & 04 & 06.8\end{array}$ & $\begin{array}{lll}39 & 12 \quad 18\end{array}$ & 1910 & 104 & 1360 & 55 & 1110 & 45 & 486 & 15 & 167 & 4 & 74 & 2 & $\mathrm{~g}$ \\
\hline $1201+396$ & $\begin{array}{lll}12 & 03 & 42.6\end{array}$ & 392255 & 310 & 43 & 230 & 10 & 160 & 30 & 63 & 2 & 18 & 1 & 6 & 1 & \\
\hline $1202+388$ & $\mid \begin{array}{lll}12 & 05 & 31.2\end{array}$ & 383403 & 680 & 52 & 400 & 17 & 300 & 31 & 114 & 3 & 37 & 5 & 24 & 1 & \\
\hline $1202+397$ & $\begin{array}{llll}12 & 04 & 48.8\end{array}$ & 392947 & & & 150 & 8 & 110 & 30 & 58 & 2 & 18 & 1 & 7 & 1 & \\
\hline $1203+384$ & $\mid \begin{array}{llll}12 & 06 & 17.1\end{array}$ & $38 \quad 1233$ & 1440 & 82 & 870 & 35 & 670 & 36 & 239 & 7 & 70 & 2 & 37 & 1 & Q \\
\hline $1204+399$ & $\mid \begin{array}{lll}12 & 06 & 37.1\end{array}$ & 394103 & 350 & 44 & 340 & 14 & 340 & 32 & 268 & 8 & 345 & 7 & 280 & 6 & Q \\
\hline $1204+401$ & $\mid \begin{array}{lll}12 & 07 & 06.2\end{array}$ & 395439 & 2390 & 126 & 1320 & 53 & 1000 & 42 & 262 & 8 & 60 & 2 & 22 & 1 & $\mathrm{G}$ \\
\hline $1205+390$ & $\mid \begin{array}{lll}12 & 07 & 57.4\end{array}$ & 384849 & & & 190 & 9 & 210 & 31 & 56 & 2 & 16 & 1 & 6 & 1 & \\
\hline $1205+392$ & $\begin{array}{llll}12 & 07 & 52.4\end{array}$ & 385558 & 3830 & 196 & 2330 & 93 & 1830 & 63 & 643 & 19 & 193 & 4 & 80 & 2 & $\mathrm{~g}$ \\
\hline $1206+399 \mathrm{~A}$ & $\begin{array}{lll}12 & 09 & 03.5\end{array}$ & $3940 \quad 16$ & & & 160 & 8 & & & 53 & 3 & 24 & 1 & 10 & 1 & $\mathrm{~g}$ \\
\hline $1206+399 B$ & 120908.5 & 393843 & & & 120 & 7 & & & 46 & 1 & 24 & 1 & 10 & 1 & $\mathrm{~g}$ \\
\hline $1206+439 \mathrm{~B}$ & 120913.5 & $43 \quad 3919$ & 12060 & 604 & 7220 & 289 & 5690 & 173 & 2005 & 60 & 602 & 12 & 262 & 5 & Q \\
\hline $1207+386$ & $12 \quad 1009.3$ & $38 \quad 21 \quad 14$ & 1850 & 101 & 1230 & 49 & 940 & 41 & 344 & 10 & 109 & 2 & 38 & 1 & G \\
\hline $1208+396$ & $\begin{array}{lll}12 & 10 & 32.5\end{array}$ & 392421 & 1550 & 87 & 1010 & 41 & 760 & 38 & 359 & 11 & 135 & 3 & 69 & 2 & $\mathrm{~g}$ \\
\hline $1209+396$ & $\mid \begin{array}{lll}12 & 12 & 27.8\end{array}$ & 392300 & 1720 & 95 & 1100 & 44 & 720 & 37 & 273 & 8 & 75 & 2 & 32 & 1 & \\
\hline $1209+399$ & $\begin{array}{llll}12 & 11 & 55.5\end{array}$ & 394249 & 400 & 45 & 260 & 11 & 190 & 31 & 68 & 2 & 19 & 1 & 7 & 1 & \\
\hline $1211+388$ & 121430.2 & $\begin{array}{llll}38 & 35 & 09\end{array}$ & 810 & 57 & 430 & 18 & 340 & 32 & 93 & 3 & 21 & 1 & 9 & 1 & \\
\hline $1212+380$ & $\mid \begin{array}{lll}12 & 14 & 56.7\end{array}$ & 374851 & 2140 & 114 & 1390 & 56 & 1080 & 44 & 302 & 9 & 57 & 2 & 23 & 1 & \\
\hline $1212+389$ & $\begin{array}{lll}12 & 15 & 13.7\end{array}$ & 383923 & 570 & 49 & 410 & 26 & 300 & 31 & 105 & 3 & 31 & 4 & 20 & 1 & Q \\
\hline $1213+389$ & $\mid \begin{array}{lll}12 & 16 & 23.4\end{array}$ & $\begin{array}{llll}38 & 38 & 17\end{array}$ & 650 & 52 & 440 & 18 & 270 & 31 & 105 & 3 & 33 & 1 & 14 & 1 & \\
\hline $1216+400$ & $\mid \begin{array}{lll}12 & 19 & 04.2\end{array}$ & 394751 & 1170 & 71 & 660 & 27 & 490 & 33 & 146 & 4 & 41 & 1 & 14 & 1 & \\
\hline $1216+402$ & $\begin{array}{llll}12 & 18 & 37.0\end{array}$ & 400046 & 1850 & 101 & 1230 & 49 & 1000 & 42 & 367 & 11 & 104 & 2 & 51 & 1 & G \\
\hline $1217+427$ & $\mid \begin{array}{lll}12 & 19 & 53.9\end{array}$ & 422950 & 1820 & 99 & 1130 & 45 & 870 & 40 & 305 & 9 & 88 & 2 & 37 & 1 & \\
\hline $1218+395$ & $\mid \begin{array}{lll}12 & 21 & 11.5\end{array}$ & $39 \quad 1846$ & 1010 & 64 & 500 & 21 & 330 & 32 & 104 & 3 & 27 & 1 & 11 & 1 & \\
\hline $1218+398$ & $12 \quad 2053.8$ & $3937 \quad 12$ & 230 & 42 & 110 & 6 & 110 & 30 & 31 & 1 & 9 & 1 & 4 & 1 & \\
\hline $1218+421$ & $\mid \begin{array}{lll}12 & 21 & 12.6\end{array}$ & 415218 & 2000 & 108 & 1310 & 53 & 1080 & 44 & 501 & 15 & 193 & 4 & 101 & 2 & $\mathrm{~g}$ \\
\hline $1219+382$ & $\begin{array}{lll}12 & 22 & 11.4\end{array}$ & 375914 & 1510 & 85 & 830 & 34 & 580 & 35 & 175 & 5 & 30 & 1 & 9 & 1 & \\
\hline $1220+393$ & 122322.5 & 390423 & 740 & 54 & 410 & 17 & 290 & 31 & 103 & 3 & 27 & 1 & 12 & 1 & \\
\hline $1220+408$ & $12 \quad 2235.2$ & 403621 & 3340 & 172 & 1960 & 79 & 1500 & 54 & 468 & 14 & 110 & 2 & 47 & 1 & \\
\hline $1221+394$ & 122410.0 & 390850 & 680 & 52 & 370 & 15 & 280 & 31 & 97 & 3 & 28 & 1 & 12 & 1 & \\
\hline $1221+397$ & $\begin{array}{lll}12 & 24 & 07.8\end{array}$ & 392956 & 410 & 45 & 220 & 10 & 120 & 30 & 65 & 2 & 24 & 1 & 11 & 1 & \\
\hline $1221+398$ & 122347.7 & 393244 & & & 210 & 10 & 130 & 30 & 76 & 2 & 25 & 1 & 14 & 1 & $\mathrm{~g}$ \\
\hline $1222+390$ & $12 \quad 2521.3$ & 384830 & 560 & 49 & 280 & 12 & 210 & 31 & 75 & 2 & 24 & 1 & 14 & 1 & \\
\hline $1222+398$ & 122431.4 & $\begin{array}{lll}39 & 32 & 17\end{array}$ & 270 & 42 & 170 & 8 & 160 & 30 & 47 & 1 & 9 & 1 & $<4$ & & \\
\hline $1222+423$ & 122428.2 & $42 \quad 0632$ & 8710 & 437 & 5130 & 205 & 4020 & 124 & 1366 & 41 & 366 & 7 & 148 & 3 & G \\
\hline $1223+395$ & 122550.5 & 391423 & 480 & 47 & 450 & 19 & 510 & 34 & 649 & 19 & 491 & 10 & 331 & 7 & G \\
\hline $1225+403$ & $\mid \begin{array}{lll}12 & 28 & 13.0\end{array}$ & $40 \quad 0413$ & 1120 & 69 & 600 & 24 & 470 & 33 & 125 & 4 & 29 & 1 & 9 & 1 & \\
\hline $1225+442$ & $12 \quad 2742.0$ & 440041 & 1680 & 93 & 1210 & 49 & 930 & 41 & 387 & 12 & 108 & 2 & 37 & 1 & $\mathrm{~g}$ \\
\hline $1226+395$ & $\mid \begin{array}{lll}12 & 29 & 12.0\end{array}$ & $39 \quad 1551$ & & & 100 & 6 & 100 & 30 & 39 & 1 & 18 & 1 & 9 & 1 & \\
\hline $1228+397$ & $\mid \begin{array}{lll}12 & 30 & 52.8\end{array}$ & 392958 & 1250 & 74 & 800 & 32 & 600 & 35 & 220 & 7 & 62 & 2 & 26 & 1 & Q \\
\hline $1228+419 \mathrm{~A}$ & 123034.6 & 413854 & 3200 & 165 & 2390 & 104 & 1770 & 61 & 838 & 25 & 295 & 6 & 155 & 3 & $\mathrm{~g}$ \\
\hline $1229+397$ & 123158.8 & 393040 & 1060 & 66 & 610 & 25 & 400 & 32 & 220 & 7 & 40 & 1 & 16 & 1 & \\
\hline $1229+405$ & $\begin{array}{llll}12 & 31 & 40.4\end{array}$ & 401732 & 1920 & 104 & 1160 & 47 & 910 & 41 & 346 & 10 & 121 & 3 & 58 & 2 & Q \\
\hline $1230+398$ & 123243.5 & 393652 & 240 & 42 & 180 & 9 & 140 & 30 & 57 & 2 & 19 & 1 & 9 & 1 & \\
\hline $1231+394$ & 123423.2 & $\begin{array}{lll}39 & 08 & 49\end{array}$ & 1330 & 78 & 650 & 26 & 470 & 33 & 133 & 4 & 33 & 1 & 15 & 1 & \\
\hline $1231+432$ & 123418.5 & 425712 & 1570 & 88 & 1000 & 40 & 820 & 39 & 311 & 9 & 94 & 2 & 41 & 1 & \\
\hline $1232+394$ & $\mid \begin{array}{lll}12 & 35 & 11.4\end{array}$ & 391059 & 830 & 58 & 450 & 19 & 300 & 31 & 93 & 3 & 21 & 1 & 11 & 1 & \\
\hline $1232+397 \mathrm{~A}$ & 123429.6 & 393035 & 1240 & 74 & 690 & 28 & 460 & 33 & 153 & 5 & 42 & 1 & 21 & 1 & G \\
\hline $1232+397 \mathrm{~B}$ & 123504.6 & 392539 & 3270 & 168 & 1630 & 65 & 1130 & 45 & 257 & 8 & 41 & 1 & 16 & 1 & G \\
\hline $1232+399$ & 123450.4 & 393844 & & & 130 & 21 & 130 & 30 & 64 & 2 & 32 & 4 & 16 & 1 & \\
\hline $1232+414 \mathrm{~A}$ & 123430.3 & 410934 & 3570 & 183 & 2660 & 108 & 1810 & 62 & 728 & 22 & 237 & 26 & 93 & 2 & $\mathrm{~g}$ \\
\hline $1233+399$ & 123615.5 & 394003 & 230 & 42 & 140 & 7 & 110 & 30 & 34 & 2 & 11 & 1 & 5 & 1 & \\
\hline $1233+418$ & $\begin{array}{lll}12 & 35 & 35.6\end{array}$ & 413707 & 2540 & 133 & 1810 & 73 & 1470 & 53 & 695 & 21 & 265 & 5 & 131 & 3 & g \\
\hline $1234+396$ & $\mid \begin{array}{lll}12 & 36 & 51.4\end{array}$ & $\begin{array}{lll}39 & 2028\end{array}$ & 740 & 54 & 620 & 25 & 540 & 34 & 348 & 10 & 234 & 5 & 171 & 4 & \\
\hline $1236+444 \mathrm{~A}$ & 123833.7 & 441343 & & & 470 & 19 & 450 & 33 & 242 & 7 & 119 & 3 & 89 & 2 & \\
\hline $1236+444 \mathrm{~B}$ & 123847.2 & 440946 & 2340 & 124 & 810 & 52 & & & 235 & 8 & 47 & 5 & 39 & 1 & $\mathrm{~g}$ \\
\hline $1239+382$ & $\mid \begin{array}{lll}12 & 42 & 12.2\end{array}$ & 375856 & 880 & 59 & 540 & 22 & 440 & 33 & 200 & 6 & 69 & 2 & 35 & 1 & $\mathrm{~g}$ \\
\hline $1239+390$ & 124133.4 & 384839 & 1810 & 99 & 970 & 39 & 750 & 37 & 249 & 7 & 69 & 2 & 28 & 1 & \\
\hline $1239+396$ & $\begin{array}{lll}12 & 41 & 47.9\end{array}$ & 392048 & 660 & 52 & 440 & 18 & 310 & 31 & 158 & 5 & 65 & 2 & 36 & 1 & $\mathrm{~g}$ \\
\hline $1239+442 \mathrm{~B}$ & $1242 \quad 19.5$ & 435611 & 2600 & 136 & 1580 & 63 & 1210 & 47 & 484 & 15 & 171 & 4 & 91 & 2 & Q \\
\hline $1240+381$ & $\begin{array}{lll}12 & 42 & 51.3\end{array}$ & 375059 & 420 & 45 & 410 & 17 & 410 & 32 & 554 & 17 & 711 & 14 & 341 & 7 & Q \\
\hline $1240+395$ & $\mid \begin{array}{lll}12 & 42 & 52.8\end{array}$ & 391546 & 1240 & 74 & 550 & 22 & 360 & 32 & 77 & 2 & 17 & 1 & 8 & 1 & \\
\hline $1241+411$ & 124420.0 & 405136 & 1340 & 78 & 900 & 36 & 800 & 38 & 370 & 11 & 145 & 3 & 74 & 2 & $\mathrm{~g}$ \\
\hline $1242+391$ & 124430.3 & 385357 & 800 & 57 & 330 & 14 & 250 & 31 & 61 & 2 & 13 & 1 & 7 & 1 & \\
\hline $1242+410$ & $\mid \begin{array}{lll}12 & 44 & 49.2\end{array}$ & 404805 & 1410 & 81 & 2140 & 86 & 2010 & 67 & 1360 & 41 & 692 & 14 & 340 & 7 & Q \\
\hline $1244+389$ & $1246 \quad 46.0$ & 384140 & 5000 & 253 & 2650 & 106 & 2170 & 72 & 599 & 18 & 143 & 3 & 52 & 1 & \\
\hline $1244+397$ & $\begin{array}{llll}12 & 47 & 19.7\end{array}$ & 392748 & 380 & 44 & 210 & 10 & 160 & 30 & 62 & 2 & 16 & 1 & 7 & 1 & \\
\hline $1245+389$ & $\mid \begin{array}{lll}12 & 48 & 20.4\end{array}$ & 384215 & 760 & 55 & 480 & 20 & 380 & 32 & 161 & 5 & 60 & 2 & 33 & 1 & \\
\hline $1245+396$ & $\begin{array}{lll}12 & 48 & 03.6\end{array}$ & 392205 & 780 & 56 & 410 & 17 & 330 & 32 & 103 & 3 & 24 & 4 & 11 & 1 & \\
\hline $1245+399$ & 12 4749.4 & 394024 & 630 & 51 & 380 & 16 & 300 & 31 & 111 & 3 & 31 & 1 & 14 & 1 & \\
\hline $1246+385 \mathrm{C}$ & 124913.5 & $38 \quad 1656$ & 1610 & 90 & 940 & 38 & 700 & 37 & 293 & 9 & 88 & 2 & 38 & 1 & \\
\hline $1247+450 \mathrm{~A}$ & 124923.2 & 444449 & 3650 & 187 & 2240 & 90 & 1680 & 59 & 689 & 21 & 216 & 4 & 97 & 2 & Q \\
\hline $1249+393$ & 125145.5 & $\begin{array}{lll}39 & 03 & 10\end{array}$ & 400 & 45 & 290 & 12 & 260 & 31 & 119 & 4 & 52 & 6 & 31 & 1 & \\
\hline $1249+432$ & 125201.0 & 425743 & 2840 & 148 & 1770 & 71 & 1400 & 52 & 562 & 17 & 169 & 4 & 69 & 2 & \\
\hline $1249+475$ & $1252 \quad 16.5$ & $47 \quad 1538$ & 8950 & 449 & 4380 & 175 & 3290 & 103 & 984 & 30 & 228 & 5 & 88 & 2 & \\
\hline $1250+384$ & 125231.6 & $\begin{array}{llll}38 & 10 & 43\end{array}$ & 1330 & 78 & 770 & 31 & 610 & 35 & 201 & 6 & 57 & 2 & 21 & 1 & $\mathrm{~g}$ \\
\hline $1250+390$ & 125249.5 & 384936 & 1110 & 68 & 600 & 24 & 460 & 33 & 154 & 5 & 40 & 1 & 14 & 1 & \\
\hline $1251+398$ & $1254 \quad 10.5$ & 393323 & 490 & 47 & 230 & 10 & 150 & 30 & 56 & 2 & 14 & 1 & 7 & 1 & Q \\
\hline
\end{tabular}


Table 2. continued

\begin{tabular}{|c|c|c|c|c|c|c|c|c|c|c|c|c|c|c|c|}
\hline B3 name & $\begin{array}{c}\mathrm{RA}(\mathrm{J} 2000) \\
{\left[\begin{array}{ccc}\mathrm{h} & \mathrm{m} & \mathrm{s}\end{array}\right]}\end{array}$ & 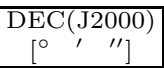 & $\begin{array}{r}S_{151} \\
{[\mathrm{mJy}]}\end{array}$ & $\begin{array}{l}\Delta S_{151} \\
{[\mathrm{mJy}]}\end{array}$ & $\begin{array}{r}S_{327} \\
{[\mathrm{mJy}]}\end{array}$ & $\begin{array}{r}S_{327} \\
{[\mathrm{mJy}]}\end{array}$ & $\begin{array}{r}S_{408} \\
{[\mathrm{mJy}]}\end{array}$ & $\begin{array}{l}\Delta S_{408} \\
{[\mathrm{mJy}]}\end{array}$ & $\begin{array}{l}S_{1400} \\
{[\mathrm{mJy}]}\end{array}$ & $\begin{array}{r}\Delta S_{1400} \\
{[\mathrm{mJy}]}\end{array}$ & $\begin{array}{l}S_{4850} \\
{[\mathrm{mJy}]}\end{array}$ & $\begin{array}{r}\Delta S_{4850} \\
{[\mathrm{mJy}]}\end{array}$ & $\begin{array}{l}S_{10550} \\
{[\mathrm{mJy}]}\end{array}$ & $\begin{array}{r}\Delta S_{10550} \\
{[\mathrm{mJy}]}\end{array}$ & Id. \\
\hline \begin{tabular}{|l|}
$1253+374$ \\
\end{tabular} & \begin{tabular}{|llll}
12 & 56 & 17.5 \\
\end{tabular} & $\begin{array}{llll}37 & 13 & 41\end{array}$ & 5260 & 266 & 2920 & 117 & 2270 & 74 & 711 & 21 & 177 & 4 & 73 & 2 & \\
\hline $1253+432$ & 125543.5 & 425825 & 3680 & 188 & 1830 & 73 & 1420 & 52 & 433 & 13 & 118 & 3 & 59 & 2 & G \\
\hline $1254+476$ & 125657.1 & 472020 & 29880 & 1495 & 16890 & 676 & 13710 & 412 & 5011 & 150 & 1674 & 33 & 727 & 15 & $\mathrm{G}$ \\
\hline $1255+448$ & $\begin{array}{llll}12 & 58 & 01.4\end{array}$ & 443522 & 7230 & 364 & 3700 & 148 & 2900 & 92 & 921 & 28 & 292 & 6 & 149 & 3 & \\
\hline $1256+392$ & 125902.4 & 390020 & 1610 & 90 & 970 & 39 & 800 & 38 & 291 & 9 & 92 & 2 & 43 & 1 & $\mathrm{Q}$ \\
\hline $1257+383$ & $\begin{array}{lll}13 & 00 & 13.7\end{array}$ & 380430 & 4650 & 236 & 2650 & 106 & 2070 & 69 & 707 & 21 & 192 & 4 & 68 & 2 & \\
\hline $1257+399$ & 125945.4 & 394037 & 300 & 43 & 130 & 7 & 110 & 30 & 32 & 1 & 10 & 1 & 4 & 1 & \\
\hline $1258+395$ & 130043.6 & $39 \quad 1904$ & 520 & 48 & 240 & 11 & 180 & 30 & 69 & 2 & 21 & 1 & 12 & 1 & \\
\hline $1258+404$ & $\begin{array}{lll}13 & 00 & 33.0\end{array}$ & 400907 & 10920 & 547 & 5690 & 228 & 4510 & 139 & 1370 & 41 & 354 & 7 & 140 & 3 & $\mathrm{Q}$ \\
\hline $1259+395$ & $\begin{array}{lll}13 & 02 & 00.6\end{array}$ & 391522 & & & 90 & 6 & 100 & 30 & 39 & 2 & 20 & 1 & 16 & 1 & $\mathrm{Q}$ \\
\hline $1300+397$ & $\begin{array}{lll}13 & 02 & 48.2\end{array}$ & 392954 & 590 & 50 & 310 & 13 & 270 & 31 & 101 & 3 & 36 & 5 & 22 & 1 & $\mathrm{Q}$ \\
\hline $1301+382$ & $\begin{array}{lll}13 & 0344.0\end{array}$ & 375609 & 3460 & 178 & 2050 & 82 & 1650 & 58 & 583 & 17 & 183 & 4 & 80 & 2 & $\mathrm{~g}$ \\
\hline $1301+393$ & $\begin{array}{lll}13 & 03 & 20.7\end{array}$ & $\begin{array}{lll}39 & 03 & 18\end{array}$ & 660 & 52 & 400 & 17 & 320 & 31 & 108 & 3 & 28 & 1 & 13 & 1 & \\
\hline $1302+388$ & 130457.9 & $3832 \quad 29$ & 4610 & 234 & 2460 & 99 & 1970 & 66 & 528 & 16 & 106 & 2 & 32 & 1 & G \\
\hline $1305+393$ & $\begin{array}{llll}13 & 07 & 37.2\end{array}$ & 390443 & 750 & 55 & 410 & 17 & 350 & 32 & 119 & 4 & 38 & 1 & 17 & 1 & \\
\hline $1306+396$ & $\begin{array}{lll}13 & 08 & 28.3\end{array}$ & 392602 & 840 & 58 & 420 & 17 & 350 & 32 & 83 & 3 & 18 & 1 & 6 & 1 & \\
\hline $1308+392$ & $\begin{array}{lll}13 & 10 & 38.2\end{array}$ & 385701 & 540 & 48 & 420 & 17 & 360 & 32 & 186 & 6 & 76 & 2 & 36 & 1 & \\
\hline $1309+412 \mathrm{~A}$ & $\begin{array}{lll}13 & 11 & 43.2\end{array}$ & 405858 & 4150 & 211 & 1880 & 96 & 1170 & 39 & 591 & 19 & 217 & 6 & 117 & 3 & $\mathrm{~g}$ \\
\hline $1311+419$ & 131404.6 & 414035 & 1970 & 106 & 1020 & 41 & 850 & 51 & 275 & 8 & 82 & 2 & 33 & 1 & \\
\hline $1312+393$ & 131506.0 & 390344 & 1430 & 82 & 840 & 34 & 710 & 37 & 249 & 7 & 84 & 2 & 40 & 1 & Q \\
\hline $1313+387$ & $\begin{array}{lll}13 & 15 & 24.2\end{array}$ & 383030 & 2950 & 153 & 1200 & 48 & 1050 & 44 & 338 & 11 & 118 & 15 & 46 & 1 & \\
\hline $1313+392$ & 131541.6 & 385833 & 420 & 45 & 320 & 14 & 280 & 31 & 151 & 5 & 68 & 2 & 29 & 1 & $\mathrm{~g}$ \\
\hline $1314+453 \mathrm{~A}$ & $\begin{array}{lll}13 & 16 & 11.9\end{array}$ & 450429 & 3160 & 163 & 1890 & 76 & 1750 & 60 & 689 & 21 & 237 & 5 & 94 & 2 & $\mathrm{G}$ \\
\hline $1315+395$ & $13 \quad 1750.2$ & $\begin{array}{lll}39 & 15 & 19\end{array}$ & 1480 & 84 & 890 & 36 & 690 & 36 & 246 & 7 & 78 & 2 & 31 & 1 & \\
\hline $1315+396$ & $13 \quad 17 \quad 18.6$ & $\begin{array}{lll}39 & 2527\end{array}$ & 2310 & 122 & 1440 & 58 & 1160 & 46 & 635 & 19 & 364 & 7 & 285 & 6 & Q \\
\hline $1317+380$ & 131952.4 & 374724 & 1570 & 88 & 900 & 36 & 750 & 37 & 276 & 8 & 101 & 2 & 47 & 1 & Q \\
\hline $1317+389$ & 132000.6 & 384022 & 210 & 41 & 280 & 12 & 310 & 31 & 287 & 9 & 139 & 13 & 81 & 2 & \\
\hline $1317+393$ & 131943.5 & 390251 & 610 & 50 & 350 & 15 & 280 & 31 & 101 & 3 & 29 & 4 & 14 & 1 & $\mathrm{G}$ \\
\hline $1318+398$ & 132027.6 & 393253 & 370 & 44 & 240 & 11 & 200 & 31 & 72 & 2 & 27 & 4 & 10 & 1 & \\
\hline $1319+388$ & 132120.1 & 383532 & 610 & 50 & 340 & 14 & 290 & 31 & 104 & 3 & 30 & 1 & 15 & 1 & \\
\hline $1319+397$ & $\begin{array}{lll}13 & 22 & 12.2\end{array}$ & 393049 & 290 & 43 & 200 & 9 & 160 & 30 & 63 & 2 & 22 & 1 & 8 & 1 & \\
\hline $1319+398$ & 132148.5 & $3938 \quad 14$ & 540 & 48 & 400 & 17 & 310 & 31 & 119 & 4 & 39 & 1 & 13 & 1 & \\
\hline $1318+428 \mathrm{C}$ & 132118.0 & 423506 & 10060 & 505 & 6190 & 248 & 4870 & 149 & 2091 & 63 & 775 & 78 & 418 & 8 & $\mathrm{~g}$ \\
\hline $1320+389$ & 132221.5 & 384404 & 560 & 49 & 260 & 11 & 200 & 31 & 64 & 2 & 23 & 1 & 10 & 1 & \\
\hline $1321+415$ & 132324.1 & $41 \quad 15 \quad 14$ & 4160 & 212 & 2290 & 92 & 1720 & 60 & 616 & 18 & 211 & 4 & 89 & 2 & $\mathrm{~g}$ \\
\hline $1322+398$ & 132444.9 & 393343 & 590 & 50 & 260 & 11 & 190 & 31 & 61 & 2 & 17 & 1 & 6 & 1 & \\
\hline $1324+390$ & 132701.8 & 384951 & 560 & 49 & 290 & 12 & 240 & 31 & 83 & 3 & 20 & 1 & 11 & 1 & \\
\hline $1324+431$ & $\begin{array}{lll}13 & 27 & 02.0\end{array}$ & 425431 & 2760 & 144 & 1410 & 57 & 1080 & 44 & 307 & 9 & 70 & 2 & 26 & 1 & \\
\hline $1327+390$ & 133004.9 & 384528 & 860 & 59 & 510 & 21 & 380 & 32 & 118 & 4 & 26 & 1 & 11 & 1 & \\
\hline $1327+391$ & 132928.6 & $3854 \quad 15$ & 550 & 49 & 290 & 12 & 230 & 31 & 65 & 2 & 16 & 1 & 7 & 1 & \\
\hline $1327+398$ & 133006.8 & $\begin{array}{lll}39 & 37 & 19\end{array}$ & 250 & 42 & 120 & 7 & 120 & 30 & 37 & 1 & 11 & 1 & 7 & 1 & \\
\hline $1327+474 \mathrm{C}$ & 132952.7 & $47 \quad 1141$ & 6900 & 347 & 3610 & 156 & 3070 & 97 & 846 & 26 & 425 & 44 & 235 & 5 & $\mathrm{~g}$ \\
\hline $1328+388$ & 133111.5 & 383500 & 430 & 45 & 470 & 19 & 420 & 33 & 253 & 8 & 89 & 2 & 33 & 1 & \\
\hline $1328+396$ & 133058.4 & $3921 \quad 17$ & 500 & 47 & 390 & 16 & 340 & 32 & 167 & 5 & 97 & 9 & 58 & 2 & \\
\hline $1330+380$ & 133249.9 & $\begin{array}{lll}37 & 45 & 13\end{array}$ & 1440 & 82 & 690 & 28 & 470 & 33 & 201 & 7 & 63 & 12 & 32 & 1 & \\
\hline $1330+389$ & 133227.0 & 384010 & 530 & 48 & 250 & 11 & 200 & 31 & 57 & 2 & 21 & 4 & 6 & 1 & \\
\hline $1330+406$ & 133255.6 & 402423 & 1590 & 89 & 890 & 36 & 690 & 36 & 228 & 7 & 54 & 2 & 19 & 1 & \\
\hline $1331+381$ & 133328.9 & 375559 & 1060 & 66 & 590 & 24 & 490 & 33 & 154 & 5 & 47 & 1 & 17 & 1 & \\
\hline $1332+385$ & 133425.3 & $\begin{array}{llll}38 & 17 & 57\end{array}$ & 880 & 59 & 560 & 23 & 510 & 34 & 245 & 7 & 102 & 2 & 54 & 1 & $\mathrm{~g}$ \\
\hline $1333+392$ & 133550.6 & 385955 & 920 & 61 & 450 & 19 & 370 & 32 & 120 & 4 & 38 & 1 & 15 & 1 & \\
\hline $1333+412$ & 133519.9 & $4100 \quad 04$ & 7460 & 375 & 3840 & 154 & 2980 & 94 & 852 & 26 & 256 & 5 & 95 & 2 & $\mathrm{~g}$ \\
\hline $1334+417$ & 133626.4 & 413112 & 1440 & 82 & 1050 & 42 & 890 & 40 & 449 & 13 & 171 & 4 & 62 & 2 & $\mathrm{G}$ \\
\hline $1336+391 \mathrm{~A}$ & 133849.7 & $3851 \quad 12$ & 20270 & 1014 & 12510 & 500 & 10000 & 301 & 3377 & 101 & 958 & 19 & 363 & 7 & $\mathrm{~g}$ \\
\hline $1336+393$ & 133815.3 & 390557 & 980 & 63 & 460 & 19 & 400 & 32 & 113 & 3 & 45 & 1 & 16 & 1 & \\
\hline $1336+396 \mathrm{C}$ & 133908.7 & 392622 & 2840 & 148 & 1540 & 62 & 1180 & 46 & 415 & 12 & 135 & 3 & 58 & 2 & \\
\hline $1336+397 \mathrm{~A}$ & 133816.3 & 392832 & & & 200 & 9 & 160 & 30 & 97 & 3 & 49 & 5 & 48 & 1 & \\
\hline $1336+397 \mathrm{~B}$ & 133855.1 & 392955 & & & 120 & 7 & & & 25 & 1 & 9 & 1 & 3 & 1 & \\
\hline $1337+385$ & 133925.1 & $\begin{array}{lll}38 & 15 & 09\end{array}$ & 900 & 60 & 510 & 21 & 440 & 33 & 111 & 3 & 34 & 1 & 8 & 1 & \\
\hline $1338+394$ & 134108.4 & $39 \quad 1451$ & 390 & 45 & 240 & 11 & 180 & 30 & 89 & 3 & 39 & 1 & 16 & 1 & Q \\
\hline $1339+438$ & 134153.4 & $43 \quad 3519$ & 2870 & 149 & 1460 & 59 & 1100 & 45 & 345 & 10 & 92 & 2 & 40 & 1 & $\mathrm{G}$ \\
\hline $1339+472$ & 134145.0 & 465716 & 4950 & 251 & 2680 & 107 & 2210 & 73 & 716 & 21 & 213 & 4 & 86 & 2 & Q \\
\hline $1340+439$ & 134306.0 & 434323 & 840 & 58 & 1340 & 54 & 1200 & 47 & 551 & 17 & 130 & 3 & 41 & 1 & \\
\hline $1341+392$ & 134320.4 & 385835 & 2980 & 154 & 1590 & 64 & 1220 & 47 & 420 & 13 & 147 & 3 & 71 & 2 & Q \\
\hline $1342+389 \mathrm{~A}$ & 134425.1 & 384129 & 2130 & 114 & 1120 & 45 & 860 & 40 & 288 & 9 & 79 & 2 & 44 & 1 & Q \\
\hline $1342+389 \mathrm{~B}$ & 134448.1 & 384436 & & & 580 & 24 & 460 & 33 & 197 & 6 & 73 & 2 & 25 & 1 & $\mathrm{~g}$ \\
\hline $1343+386$ & 134536.9 & $\begin{array}{lll}38 & 23 & 12\end{array}$ & 1340 & 78 & 1810 & 73 & 1520 & 55 & 899 & 27 & 396 & 8 & 195 & 4 & $\mathrm{Q}$ \\
\hline $1343+430$ & 134533.1 & 425016 & 4790 & 243 & 3240 & 130 & 2720 & 87 & 1154 & 35 & 403 & 8 & 160 & 3 & G \\
\hline $1344+397$ & 134704.3 & 392858 & 390 & 45 & 260 & 11 & 160 & 30 & 104 & 3 & 78 & 2 & 77 & 2 & \\
\hline $1345+398$ & $1347 \quad 13.5$ & 393558 & 400 & 45 & 250 & 11 & 180 & 30 & 65 & 2 & 20 & 1 & 7 & 1 & \\
\hline $1346+392$ & 134856.9 & $\begin{array}{lll}39 & 00 & 43\end{array}$ & 440 & 46 & 270 & 12 & 220 & 31 & 85 & 3 & 28 & 1 & 14 & 1 & \\
\hline $1347+391$ & 134925.7 & 385142 & 580 & 49 & 470 & 19 & 440 & 33 & 180 & 5 & 64 & 2 & 32 & 1 & \\
\hline $1347+396$ & 134909.2 & 392306 & 540 & 48 & 450 & 19 & 380 & 32 & 179 & 5 & 69 & 2 & 29 & 1 & \\
\hline $1347+398$ & 134915.9 & 393647 & 930 & 61 & 520 & 21 & 390 & 32 & 148 & 4 & 48 & 1 & 22 & 1 & \\
\hline $1347+403$ & 134952.1 & 400621 & 810 & 57 & 510 & 21 & 430 & 33 & 130 & 4 & 35 & 1 & 13 & 1 & \\
\hline $1348+392$ & 135032.4 & 385922 & 1320 & 77 & 670 & 27 & 500 & 34 & 169 & 5 & 86 & 2 & 101 & 2 & Q \\
\hline $1348+396$ & 135039.8 & 392125 & 510 & 47 & 300 & 13 & 220 & 31 & 101 & 3 & 32 & 4 & 27 & 1 & \\
\hline $1349+394$ & 135149.6 & 391344 & & & 100 & 6 & 100 & 30 & 60 & 4 & 7 & 2 & 12 & 1 & \\
\hline $1349+388$ & 135119.8 & $38 \quad 3814$ & 300 & 43 & 220 & 10 & 220 & 31 & 251 & 8 & 164 & 3 & 116 & 3 & $\mathrm{Q}$ \\
\hline $1350+395$ & $\begin{array}{llll}13 & 52 & 29.4 \\
\end{array}$ & $\begin{array}{llll}39 & 18 & 36 \\
\end{array}$ & 850 & 58 & 410 & 17 & 290 & 31 & 84 & 3 & 21 & 4 & 7 & 1 & \\
\hline
\end{tabular}


Table 2. continued

\begin{tabular}{|c|c|c|c|c|c|c|c|c|c|c|c|c|c|c|c|}
\hline B3 name & $\begin{array}{l}\mathrm{RA}(\mathrm{J} 2000) \\
{\left[\begin{array}{ccc}\mathrm{h} & \mathrm{m} & \mathrm{s}\end{array}\right]} \\
\end{array}$ & 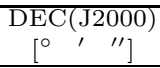 & $\begin{array}{r}S_{151} \\
{[\mathrm{mJy}]}\end{array}$ & $\begin{array}{r}\Delta S_{151} \\
{[\mathrm{mJy}]}\end{array}$ & $\begin{array}{r}S_{327} \\
{[\mathrm{mJy}]}\end{array}$ & $\begin{array}{r}S_{327} \\
{[\mathrm{mJy}]}\end{array}$ & $\begin{array}{r}S_{408} \\
{[\mathrm{mJy}]}\end{array}$ & $\begin{array}{l}\Delta S_{408} \\
{[\mathrm{mJy}]}\end{array}$ & $\begin{array}{l}S_{1400} \\
{[\mathrm{mJy}]}\end{array}$ & $\begin{array}{r}\Delta S_{1400} \\
{[\mathrm{mJy}]}\end{array}$ & $\begin{array}{l}S_{4850} \\
{[\mathrm{mJy}]}\end{array}$ & $\begin{array}{r}S_{4850} \\
{[\mathrm{mJy}]}\end{array}$ & $\begin{array}{r}S_{10550} \\
{[\mathrm{mJy}]}\end{array}$ & $\begin{array}{r}\Delta S_{10550} \\
{[\mathrm{mJy}]}\end{array}$ & Id. \\
\hline $1350+432$ & $1352 \quad 28.5$ & 425923 & 2980 & 154 & 1210 & 49 & 830 & 39 & 170 & $\frac{5}{5}$ & 33 & 1 & 10 & 1 & $\mathrm{G}$ \\
\hline $1352+383$ & $\begin{array}{lll}13 & 55 & 03.2\end{array}$ & 380454 & 1070 & 67 & 590 & 24 & 440 & 33 & 145 & 4 & 36 & 1 & 18 & 1 & \\
\hline $1352+397$ & 135411.3 & 392756 & 920 & 61 & 460 & 19 & 320 & 31 & 125 & 4 & 29 & 4 & 16 & 1 & \\
\hline $1352+403$ & $1354 \quad 07.3$ & 400556 & 1070 & 67 & 580 & 24 & 470 & 33 & 160 & 5 & 58 & 2 & 25 & 1 & \\
\hline $1353+380$ & 135553.7 & 374524 & 990 & 64 & 640 & 26 & 490 & 33 & 180 & 5 & 60 & 2 & 24 & 1 & \\
\hline $1353+397$ & $\begin{array}{lll}13 & 56 & 03.4\end{array}$ & $\begin{array}{lll}39 & 33 & 23\end{array}$ & & & 740 & 30 & 530 & 34 & 182 & 5 & 39 & 1 & 14 & 1 & \\
\hline $1354+397$ & 135615.9 & 392924 & 3500 & 180 & 1440 & 58 & 960 & 42 & 226 & 7 & 35 & 1 & 12 & 1 & \\
\hline $1355+380$ & 135739.9 & 374942 & 1260 & 75 & 720 & 29 & 600 & 35 & 217 & 7 & 85 & 2 & 71 & 2 & Q \\
\hline $1356+393$ & 135847.8 & 390402 & 1260 & 75 & 900 & 36 & 740 & 37 & 300 & 9 & 93 & 2 & 41 & 1 & \\
\hline $1356+397$ & $1358 \quad 15.5$ & 393247 & & & 130 & 7 & 120 & 30 & 37 & 1 & 15 & 1 & 9 & 1 & $\mathrm{Q}$ \\
\hline $1357+392$ & 135912.3 & 390033 & 570 & 49 & 290 & 12 & 200 & 31 & 61 & 2 & 19 & 1 & 7 & 1 & $\mathrm{~b}$ \\
\hline $1357+394 \mathrm{~A}$ & 135955.4 & $39 \quad 1304$ & & & 150 & 8 & 210 & 31 & 185 & 6 & 39 & 1 & 14 & 1 & \\
\hline $1357+394 B$ & $\begin{array}{lll}14 & 00 & 03.1\end{array}$ & 391053 & & & 520 & 21 & 410 & 32 & 146 & 4 & 54 & 2 & 23 & 1 & Q \\
\hline $1358+433$ & $\begin{array}{llll}14 & 00 & 32.1\end{array}$ & 430403 & 3380 & 174 & 1800 & 72 & 1370 & 51 & 431 & 13 & 119 & 3 & 42 & 1 & \\
\hline $1359+419$ & 140133.8 & 414215 & 2690 & 140 & 1520 & 61 & 1160 & 46 & 390 & 12 & 111 & 2 & 49 & 1 & G \\
\hline $1401+387$ & $\begin{array}{llll}14 & 03 & 11.8\end{array}$ & 382751 & 3330 & 171 & 2090 & 84 & 1640 & 58 & 670 & 20 & 211 & 4 & 114 & 2 & \\
\hline $1401+395$ & $\begin{array}{lll}14 & 04 & 04.9\end{array}$ & $\begin{array}{lll}39 & 2109\end{array}$ & 450 & 46 & 250 & 11 & 170 & 30 & 58 & 2 & 19 & 1 & 6 & 1 & \\
\hline $1402+382$ & 140428.0 & $\begin{array}{lll}38 & 00 & 29\end{array}$ & 1700 & 94 & 850 & 34 & 640 & 36 & 201 & 6 & 58 & 2 & 20 & 1 & \\
\hline $1403+395$ & $\begin{array}{llll}14 & 05 & 12.1\end{array}$ & $\begin{array}{llll}39 & 17 & 22\end{array}$ & 620 & 51 & 320 & 14 & 240 & 31 & 72 & 2 & 21 & 1 & 8 & 1 & \\
\hline $1406+397$ & 140825.0 & 393131 & 810 & 57 & 440 & 18 & 320 & 31 & 112 & 3 & 31 & 4 & 18 & 1 & \\
\hline $1407+388$ & $\begin{array}{lll}14 & 09 & 27.0\end{array}$ & $\begin{array}{llll}38 & 38 & 01\end{array}$ & 520 & 48 & 320 & 14 & 260 & 31 & 117 & 4 & 44 & 1 & 23 & 1 & $\mathrm{~g}$ \\
\hline $1408+398$ & $14 \quad 1041.3$ & 393436 & 1030 & 65 & 540 & 22 & 420 & 33 & 151 & 5 & 47 & 1 & 16 & 1 & \\
\hline $1408+399$ & $\begin{array}{llll}14 & 10 & 17.6\end{array}$ & 394525 & 370 & 44 & 190 & 9 & 170 & 30 & 63 & 3 & 17 & 1 & 4 & 1 & \\
\hline $1409+390$ & $\begin{array}{lll}14 & 11 & 42.4\end{array}$ & $\begin{array}{lll}38 & 46 & 19\end{array}$ & 880 & 59 & 550 & 22 & 460 & 33 & 191 & 6 & 62 & 2 & 29 & 1 & \\
\hline $1409+394$ & $\begin{array}{lll}14 & 11 & 26.9\end{array}$ & $\begin{array}{llll}39 & 14 & 17\end{array}$ & 660 & 52 & 380 & 16 & 290 & 31 & 124 & 4 & 43 & 1 & 15 & 1 & \\
\hline $1410+438$ & $\begin{array}{lll}14 & 12 & 17.4\end{array}$ & 433752 & 3390 & 174 & 1770 & 71 & 1340 & 50 & 474 & 14 & 123 & 3 & 56 & 1 & \\
\hline $1411+391 \mathrm{~A}$ & $\begin{array}{lll}14 & 13 & 52.9\end{array}$ & 385328 & & & 230 & 10 & 220 & 31 & 93 & 3 & 42 & 1 & 19 & 1 & \\
\hline $1411+391 B$ & $\begin{array}{lll}14 & 14 & 00.2\end{array}$ & 385526 & & & 520 & 21 & 390 & 32 & 190 & 6 & 103 & 2 & 38 & 1 & \\
\hline $1411+397$ & $\begin{array}{lll}14 & 13 & 12.9\end{array}$ & 393056 & 390 & 45 & 190 & 9 & 130 & 30 & 76 & 2 & 33 & 1 & 14 & 1 & $\mathrm{~g}$ \\
\hline $1411+427$ & 141340.8 & 422923 & 2240 & 119 & 1270 & 51 & 960 & 42 & 330 & 10 & 96 & 2 & 38 & 1 & \\
\hline $1412+392$ & $\begin{array}{lll}14 & 15 & 03.3\end{array}$ & $\begin{array}{lll}38 & 59 & 16\end{array}$ & 780 & 56 & 600 & 24 & 470 & 33 & 234 & 7 & 94 & 2 & 54 & 1 & \\
\hline $1412+397$ & 141450.0 & 393005 & 390 & 45 & 200 & 9 & 170 & 30 & 47 & 2 & & & 6 & 1 & \\
\hline $1413+398$ & 141515.9 & 393742 & 450 & 46 & 170 & 8 & 130 & 30 & 35 & 2 & 9 & 1 & 4 & 1 & \\
\hline $1414+398$ & 141632.8 & 393634 & 950 & 62 & 370 & 15 & 240 & 31 & 102 & 3 & 29 & 4 & 11 & 1 & $\mathrm{~g}$ \\
\hline $1415+391$ & $1417 \quad 54.5$ & 385803 & 400 & 45 & 250 & 11 & 210 & 31 & 82 & 4 & 25 & 1 & 10 & 1 & \\
\hline $1416+400$ & $14 \quad 18 \quad 58.8$ & 394639 & 1550 & 87 & 840 & 34 & 670 & 36 & 256 & 8 & 115 & 3 & 74 & 2 & Q \\
\hline $1417+383$ & 141955.4 & 380625 & 1040 & 66 & 690 & 28 & 540 & 34 & 209 & 6 & 66 & 2 & 30 & 1 & \\
\hline $1417+385$ & 141946.5 & 382148 & 410 & 45 & 600 & 24 & 470 & 33 & 624 & 19 & 1371 & 27 & 899 & 18 & Q \\
\hline $1417+397$ & 141948.8 & $3932 \quad 20$ & 250 & 42 & 150 & 8 & 120 & 30 & 52 & 2 & 15 & 1 & 7 & 1 & \\
\hline $1418+388$ & 142101.7 & 383544 & 1240 & 74 & 700 & 28 & 550 & 34 & 144 & 4 & 31 & 1 & 10 & 1 & \\
\hline $1419+397$ & 142123.5 & $3933 \quad 31$ & 480 & 47 & 740 & 30 & 570 & 35 & 395 & 12 & 187 & 4 & 98 & 2 & $\mathrm{~g}$ \\
\hline $1419+399$ & 142125.6 & 394328 & 290 & 43 & 180 & 9 & 140 & 30 & 91 & 4 & 57 & 6 & 56 & 2 & Q \\
\hline $1419+419$ & 142105.8 & 414449 & 15820 & 792 & 9890 & 396 & 8170 & 247 & 3097 & 93 & 917 & 18 & 372 & 8 & $\mathrm{~g}$ \\
\hline $1420+386$ & 142213.6 & 382654 & 760 & 55 & 510 & 21 & 410 & 32 & 169 & 5 & 60 & 2 & 26 & 1 & \\
\hline $1422+395$ & 142422.4 & 392144 & 970 & 63 & 510 & 21 & & & 172 & 6 & 58 & 12 & 33 & 1 & $\mathrm{~g}$ \\
\hline $1422+401 B$ & 142428.8 & $\begin{array}{lll}39 & 5319\end{array}$ & 1530 & 86 & 920 & 37 & 740 & 37 & 287 & 9 & 90 & 2 & 45 & 1 & \\
\hline $1424+380$ & 142605.9 & 374858 & 5570 & 281 & 1480 & 59 & 780 & 38 & 48 & 3 & 5 & 1 & $<3$ & & \\
\hline $1426+394$ & 142813.9 & $\begin{array}{lll}39 & 12 & 19\end{array}$ & 900 & 60 & 530 & 22 & 440 & 33 & 272 & 8 & 143 & 3 & 155 & 3 & \\
\hline $1426+398$ & 142845.7 & 393838 & 990 & 64 & 420 & 17 & 270 & 31 & 90 & 5 & 9 & 2 & 6 & 1 & \\
\hline $1427+404$ & 142956.9 & 401117 & 830 & 58 & 480 & 20 & 410 & 32 & 178 & 5 & 54 & 2 & 29 & 1 & $\mathrm{~g}$ \\
\hline $1428+380$ & 143034.4 & 375010 & 1050 & 66 & 650 & 26 & 520 & 34 & 208 & 6 & 77 & 2 & 40 & 1 & $\mathrm{~g}$ \\
\hline $1428+385$ & 143055.2 & 381936 & 2010 & 108 & 1180 & 47 & 820 & 39 & 300 & 9 & 85 & 2 & 36 & 1 & \\
\hline $1429+392$ & $\begin{array}{lll}14 & 31 & 36.7\end{array}$ & 385935 & 510 & 47 & 410 & 17 & 310 & 31 & 108 & 3 & 20 & 1 & 8 & 1 & \\
\hline $1429+395$ & 143142.5 & 391905 & 930 & 61 & 550 & 22 & 380 & 32 & 157 & 5 & 51 & 1 & 19 & 1 & \\
\hline $1430+399$ & $1432 \quad 27.0$ & 394450 & & & 120 & 7 & 140 & 30 & 45 & 2 & 9 & 1 & 7 & 1 & \\
\hline $1432+382$ & 143457.5 & 380450 & 2650 & 138 & 1750 & 70 & 1090 & 44 & 463 & 14 & 166 & 3 & 72 & 2 & $\mathrm{~g}$ \\
\hline $1432+389$ & $1434 \quad 14.4$ & 384257 & 490 & 47 & 280 & 12 & 220 & 31 & 71 & 2 & 28 & 4 & 9 & 1 & \\
\hline $1432+397 \mathrm{~A}$ & 143419.3 & 392933 & & & 100 & 6 & 100 & 30 & 52 & 2 & 10 & 1 & $<3$ & & \\
\hline $1432+397 \mathrm{~B}$ & 143456.4 & 393409 & 300 & 43 & 200 & 9 & 150 & 30 & 73 & 2 & 29 & 1 & 16 & 1 & $\mathrm{~g}$ \\
\hline $1432+428 \mathrm{~B}$ & 143427.9 & $42 \quad 36 \quad 19$ & 1030 & 65 & 940 & 38 & 930 & 41 & 897 & 27 & 356 & 7 & 183 & 4 & \\
\hline $1435+383$ & 143733.4 & 380745 & 1130 & 69 & 710 & 29 & 540 & 34 & 217 & 7 & 69 & 2 & 48 & 1 & Q \\
\hline $1435+429$ & 143653.8 & $4244 \quad 16$ & 2560 & 134 & 1540 & 62 & 920 & 41 & 272 & 8 & 107 & 2 & 26 & 1 & \\
\hline $1436+399$ & 143839.6 & 394259 & 450 & 46 & 220 & 10 & 130 & 30 & 46 & 1 & 10 & 1 & 7 & 1 & \\
\hline $1437+397$ & 143910.6 & $3933 \quad 35$ & 480 & 47 & 350 & 15 & 240 & 31 & 96 & 4 & 59 & 2 & 16 & 1 & \\
\hline $1437+427$ & 143946.2 & 423427 & 5580 & 282 & 2560 & 103 & 1910 & 65 & 528 & 16 & 128 & 3 & 56 & 2 & G \\
\hline $1438+382$ & $\begin{array}{lll}14 & 40 & 11.7\end{array}$ & 380156 & 1250 & 74 & 850 & 34 & 670 & 36 & 216 & 7 & 44 & 1 & 14 & 1 & \\
\hline $1438+385$ & 144022.2 & $\begin{array}{lll}38 & 20 & 16\end{array}$ & 1230 & 73 & 1130 & 45 & 960 & 42 & 1077 & 32 & 789 & 16 & 640 & 13 & Q \\
\hline $1438+406$ & 144028.0 & 402828 & 1050 & 66 & 580 & 24 & 400 & 32 & 146 & 4 & 50 & 1 & 18 & 1 & $\mathrm{~g}$ \\
\hline $1441+409$ & 144259.4 & 404428 & 1330 & 78 & 1740 & 70 & 1680 & 59 & 985 & 30 & 323 & 7 & 144 & 3 & \\
\hline $1442+383$ & $1444 \quad 17.1$ & $3808 \quad 34$ & 720 & 54 & 520 & 21 & 420 & 33 & 175 & 5 & 64 & 2 & 26 & 1 & \\
\hline $1442+384$ & 144439.5 & $\begin{array}{llll}38 & 17 & 17\end{array}$ & 1180 & 71 & 650 & 26 & 460 & 33 & 152 & 5 & 35 & 1 & 23 & 1 & \\
\hline $1442+441$ & 144449.5 & 435742 & 3370 & 173 & 1710 & 69 & 1240 & 48 & 397 & 12 & 99 & 2 & 38 & 1 & \\
\hline $1444+395$ & 144627.5 & 392100 & 360 & 44 & 210 & 10 & 150 & 30 & 63 & 2 & 26 & 1 & 10 & 1 & \\
\hline $1444+417 \mathrm{~A}$ & 144627.2 & 413319 & 3930 & 201 & 2130 & 85 & 1580 & 56 & 535 & 16 & 131 & 16 & 72 & 2 & Q \\
\hline $1445+410$ & $\begin{array}{lll}14 & 47 & 12.7\end{array}$ & 404745 & 1820 & 99 & 1130 & 45 & 980 & 42 & 412 & 12 & 165 & 3 & 102 & 2 & $\mathrm{~g}$ \\
\hline $1446+399$ & $\begin{array}{lll}14 & 48 & 07.4\end{array}$ & 394420 & 750 & 55 & 440 & 18 & & & 103 & 5 & 10 & 2 & 11 & 1 & \\
\hline $1446+440$ & 144832.0 & 435235 & 3910 & 200 & 2050 & 82 & 1420 & 52 & 543 & 16 & 154 & 3 & 70 & 2 & \\
\hline $1447+380$ & $\begin{array}{lll}14 & 49 & 17.0\end{array}$ & $37 \quad 48 \quad 42$ & 1260 & 75 & 660 & 27 & 420 & 33 & 114 & 3 & 27 & 1 & 10 & 1 & \\
\hline
\end{tabular}


Table 2. continued

\begin{tabular}{|c|c|c|c|c|c|c|c|c|c|c|c|c|c|c|c|}
\hline B3 name & \begin{tabular}{|c|c|}
$\mathrm{RA}(\mathrm{J} 2000)$ \\
{$\left[\begin{array}{ccc}\mathrm{h} & \mathrm{m} & \mathrm{s}\end{array}\right]$}
\end{tabular} & 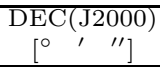 & $\begin{array}{r}S_{151} \\
{[\mathrm{mJy}]} \\
\end{array}$ & $\begin{array}{r}\Delta S_{151} \\
{[\mathrm{mJy}]}\end{array}$ & $\begin{array}{r}S_{327} \\
{[\mathrm{mJy}]}\end{array}$ & $\begin{array}{r}S_{327} \\
{[\mathrm{mJy}]}\end{array}$ & $\begin{array}{r}S_{408} \\
{[\mathrm{mJy}]}\end{array}$ & $\begin{array}{l}\Delta S_{408} \\
{[\mathrm{mJy}]}\end{array}$ & $\begin{array}{l}S_{1400} \\
{[\mathrm{mJy}]}\end{array}$ & $\begin{array}{r}\Delta S_{1400} \\
{[\mathrm{mJy}]}\end{array}$ & $\begin{array}{l}S_{4850} \\
{[\mathrm{mJy}]}\end{array}$ & $\begin{array}{r}S_{4850} \\
{[\mathrm{mJy}]}\end{array}$ & $\begin{array}{r}S_{10550} \\
{[\mathrm{mJy}]}\end{array}$ & $\begin{array}{r}\Delta S_{10550} \\
{[\mathrm{mJy}]}\end{array}$ & Id. \\
\hline \begin{tabular}{|l|}
$1447+400$ \\
\end{tabular} & $\begin{array}{|llll|}14 & 49 & 29.6 \\
\end{array}$ & $3948 \quad 23$ & 2310 & 122 & 1330 & 53 & 970 & 42 & 322 & 10 & 102 & 2 & 50 & 1 & \\
\hline $1447+402$ & 144902.3 & 400021 & 2300 & 122 & 1730 & 73 & 1120 & 45 & 501 & 15 & 178 & 4 & 104 & 2 & $\mathrm{~g}$ \\
\hline $1449+380$ & 145059.8 & 375131 & 1070 & 67 & 690 & 28 & 520 & 34 & 193 & 6 & 51 & 1 & 17 & 1 & \\
\hline $1449+421$ & 145107.3 & 415442 & 1010 & 64 & 2580 & 103 & 2370 & 77 & 815 & 24 & 138 & 3 & 41 & 1 & \\
\hline $1450+391 B$ & 145205.8 & $\begin{array}{lll}38 & 58 & 19\end{array}$ & 3390 & 174 & 2320 & 101 & 1680 & 59 & 568 & 17 & 136 & 17 & 72 & 2 & $\mathrm{~g}$ \\
\hline $1450+396$ & 145203.7 & 392552 & 350 & 44 & 180 & 9 & 110 & 30 & 45 & 1 & 18 & 1 & 7 & 1 & \\
\hline $1451+396$ & 145349.3 & 392549 & 710 & 53 & 430 & 18 & 320 & 31 & 107 & 3 & 28 & 4 & 8 & 1 & \\
\hline $1452+394$ & 145455.5 & 391151 & 410 & 45 & 310 & 13 & 240 & 31 & 117 & 4 & 46 & 5 & 24 & 1 & $\mathrm{~g}$ \\
\hline $1453+397$ & 145530.0 & 393358 & 750 & 55 & 420 & 17 & 290 & 31 & 87 & 3 & 21 & 1 & 9 & 1 & \\
\hline $1454+394$ & 145620.0 & 391445 & 570 & 49 & 350 & 15 & 260 & 31 & 86 & 3 & 24 & 1 & 12 & 1 & G \\
\hline $1455+399$ & 145747.0 & 394560 & & & 100 & 6 & 100 & 30 & 23 & 1 & 7 & 1 & 2 & 1 & \\
\hline $1455+421$ & $1457 \quad 40.7$ & 415850 & 2920 & 151 & 1680 & 67 & 1390 & 51 & 517 & 16 & 203 & 4 & 115 & 3 & G \\
\hline $1457+388 \mathrm{~A}$ & 145911.0 & 383637 & 1180 & 71 & 660 & 27 & 510 & 34 & 135 & 4 & 36 & 1 & 12 & 1 & \\
\hline $1458+433$ & $\begin{array}{llll}15 & 00 & 29.9\end{array}$ & 430951 & 2010 & 108 & 1270 & 51 & 1080 & 44 & 440 & 13 & 143 & 3 & 62 & 2 & G \\
\hline $1459+399$ & $\begin{array}{llll}15 & 01 & 09.4\end{array}$ & 394241 & 730 & 54 & 400 & 17 & & & 118 & 4 & 38 & 8 & 19 & 1 & \\
\hline $2300+382$ & $2303 \quad 15.0$ & 382838 & 1760 & 97 & 830 & 34 & 610 & 35 & 229 & 7 & 83 & 2 & 34 & 1 & $\mathrm{~g}$ \\
\hline $2301+394 \mathrm{~A}$ & 230348.5 & 394124 & & & 560 & 23 & & & 162 & 8 & 91 & 2 & 25 & 1 & \\
\hline $2301+394 \mathrm{~B}$ & 230356.8 & $\begin{array}{lll}39 & 4307\end{array}$ & & & 390 & 16 & 330 & 32 & 125 & 4 & 43 & 1 & 19 & 1 & \\
\hline $2301+398$ & $\mid \begin{array}{lll}23 & 03 & 26.7\end{array}$ & 400614 & 650 & 52 & 390 & 16 & 370 & 32 & 90 & 4 & 33 & 1 & 9 & 1 & G \\
\hline $2301+430$ & $2303 \quad 47.3$ & 431660 & 2910 & 151 & 1290 & 52 & 1010 & 43 & 274 & 8 & 67 & 2 & 36 & 1 & \\
\hline $2301+443$ & $\begin{array}{lll}23 & 03 & 45.3\end{array}$ & 443906 & 7280 & 366 & 4520 & 181 & 4040 & 125 & 1236 & 37 & 261 & 5 & 61 & 2 & \\
\hline $2302+402$ & 230454.8 & 402853 & 3740 & 191 & 2790 & 112 & 2530 & 82 & 1183 & 35 & 408 & 8 & 160 & 3 & \\
\hline $2303+391 \mathrm{~A}$ & 230604.7 & 392708 & 4140 & 211 & 2240 & 90 & 1850 & 63 & 777 & 23 & 244 & 26 & 151 & 3 & $\mathrm{~g}$ \\
\hline $2304+377$ & $\begin{array}{llll}23 & 07 & 01.0\end{array}$ & $\begin{array}{lll}38 & 02 & 42\end{array}$ & 2720 & 142 & 2670 & 107 & 2600 & 84 & 1496 & 45 & 519 & 10 & 234 & 5 & $\mathrm{~g}$ \\
\hline $2304+398$ & $\begin{array}{llll}23 & 07 & 03.2\end{array}$ & 400959 & 380 & 44 & 220 & 10 & 200 & 31 & 87 & 4 & 28 & 4 & 13 & 1 & \\
\hline $2304+429$ & $\begin{array}{llll}23 & 06 & 32.0\end{array}$ & $43 \quad 1047$ & 2080 & 111 & 1100 & 44 & 840 & 39 & 315 & 9 & 97 & 2 & 36 & 1 & \\
\hline $2305+404$ & \begin{tabular}{|llll}
23 & 07 & 53.7
\end{tabular} & 404149 & 3180 & 164 & 1360 & 55 & 990 & 42 & 247 & 7 & 58 & 2 & 16 & 1 & G \\
\hline $2306+392$ & 230856.0 & 393335 & 600 & 50 & 310 & 13 & 220 & 31 & 75 & 2 & 28 & 1 & 11 & 1 & \\
\hline $2308+393$ & $\begin{array}{llll}23 & 10 & 57.2\end{array}$ & 393839 & 810 & 57 & 320 & 14 & 240 & 31 & 107 & 3 & 32 & 4 & 15 & 1 & \\
\hline $2308+395$ & $\begin{array}{llll}23 & 10 & 41.0\end{array}$ & 395142 & & & 100 & 6 & 110 & 30 & 37 & 1 & 15 & 1 & 7 & 1 & \\
\hline $2308+400$ & $23 \quad 10 \quad 54.2$ & 402009 & 1640 & 91 & 780 & 32 & 630 & 35 & 188 & 6 & 49 & 1 & 17 & 1 & \\
\hline $2311+387$ & 231400.3 & 390150 & 680 & 52 & 620 & 25 & 500 & 34 & 251 & 8 & 98 & 2 & 32 & 1 & \\
\hline $2311+396 \mathrm{~A}$ & $23 \quad 1350.3$ & 395303 & & & 130 & 7 & 120 & 30 & 32 & 2 & 11 & 1 & $<3$ & & $\mathrm{~F}$ \\
\hline $2311+396 B$ & $\begin{array}{llll}23 & 14 & 16.3\end{array}$ & 395512 & & & 130 & 7 & 130 & 30 & 39 & 1 & 10 & 1 & 4 & 1 & \\
\hline $2311+469$ & $23 \quad 1348.2$ & $47 \quad 12 \quad 16$ & 9330 & 468 & 5210 & 208 & 4340 & 134 & 1942 & 58 & 716 & 14 & 244 & 5 & $\mathrm{Q}$ \\
\hline $2313+406$ & \begin{tabular}{|lll}
23 & 16 & 09.7
\end{tabular} & 405517 & 1750 & 96 & 870 & 35 & 680 & 36 & 218 & 7 & 66 & 2 & 26 & 1 & \\
\hline $2315+396$ & $\begin{array}{llll}23 & 17 & 48.4\end{array}$ & 395237 & 5680 & 287 & 3280 & 131 & 2500 & 81 & 1052 & 32 & 341 & 7 & 212 & 4 & $\mathrm{~g}$ \\
\hline $2316+398$ & $\begin{array}{llll}23 & 19 & 20.9\end{array}$ & 401008 & 920 & 61 & 350 & 15 & 290 & 31 & 109 & 3 & 40 & 5 & 22 & 1 & $\mathrm{Q}$ \\
\hline $2318+389$ & $\begin{array}{llll}23 & 21 & 19.2\end{array}$ & 391215 & 560 & 49 & 300 & 13 & 220 & 31 & 81 & 2 & 20 & 1 & 10 & 1 & \\
\hline $2320+396$ & $\begin{array}{lll}23 & 22 & 44.4\end{array}$ & 395801 & 560 & 49 & 330 & 14 & 260 & 31 & 107 & 3 & 52 & 6 & 27 & 1 & \\
\hline $2320+416 B$ & $23 \quad 2245.3$ & 415839 & 2480 & 130 & 1250 & 50 & 1070 & 44 & 407 & 13 & 141 & 17 & 68 & 2 & $\mathrm{~g}$ \\
\hline $2321+423$ & 232354.2 & 423548 & 4130 & 210 & 2610 & 105 & 2070 & 69 & 899 & 27 & 299 & 6 & 96 & 2 & G \\
\hline $2322+396$ & $\begin{array}{llll}23 & 25 & 17.9\end{array}$ & 395736 & & & 150 & 8 & 120 & 30 & 129 & 4 & 95 & 2 & 117 & 3 & $\mathrm{~F}$ \\
\hline $2322+403$ & 232448.5 & 404021 & 1700 & 94 & 1060 & 43 & 850 & 39 & 341 & 10 & 95 & 2 & 33 & 1 & \\
\hline $2323+388$ & $\begin{array}{lll}23 & 25 & 40.2\end{array}$ & 390555 & 1200 & 72 & 640 & 26 & 480 & 33 & 202 & 6 & 67 & 2 & 31 & 1 & \\
\hline $2323+398$ & $2325 \quad 31.3$ & $40 \quad 08 \quad 19$ & 590 & 50 & 220 & 10 & 170 & 30 & 47 & 2 & 11 & 1 & 5 & 1 & \\
\hline $2323+435 \mathrm{~A}$ & 232542.3 & 434658 & 5520 & 279 & 3280 & 131 & 2900 & 92 & 1754 & 53 & 944 & 19 & 368 & 7 & $\mathrm{~g}$ \\
\hline $2324+394 B$ & 232708.2 & $3943 \quad 19$ & 480 & 47 & 210 & 10 & & & 55 & 2 & 26 & 4 & 10 & 1 & \\
\hline $2324+405$ & 232655.7 & $4048 \quad 07$ & 10490 & 526 & 6640 & 266 & 5520 & 168 & 2434 & 73 & 983 & 20 & 442 & 9 & \\
\hline $2325+396$ & $\begin{array}{lll}23 & 27 & 58.9\end{array}$ & 395542 & 780 & 56 & 370 & 15 & 280 & 31 & 118 & 4 & 40 & 5 & 15 & 1 & \\
\hline $2326+395$ & $23 \quad 2850.9$ & 394757 & 1240 & 74 & 730 & 30 & 510 & 34 & 208 & 6 & 66 & 2 & 22 & 1 & \\
\hline $2326+422$ & $2328 \quad 58.2$ & 423203 & 2220 & 118 & 1190 & 48 & 1020 & 43 & 450 & 14 & 176 & 4 & 96 & 2 & $\mathrm{~g}$ \\
\hline $2327+391$ & $\begin{array}{llll}23 & 30 & 24.9\end{array}$ & $\begin{array}{lll}39 & 27 & 12\end{array}$ & 530 & 48 & 450 & 19 & 360 & 32 & 100 & 3 & 32 & 1 & 14 & 1 & $\mathrm{G}$ \\
\hline $2327+407$ & 233008.6 & 410425 & 960 & 62 & 570 & 23 & 480 & 33 & 227 & 7 & 168 & 4 & 83 & 2 & Q \\
\hline $2327+422$ & 233005.0 & 423346 & 3190 & 164 & 1550 & 62 & 1120 & 45 & 378 & 11 & 101 & 2 & 46 & 1 & \\
\hline $2328+397$ & 233126.5 & 400332 & & & 70 & 5 & 110 & 30 & 19 & 1 & 5 & 1 & 5 & 1 & \\
\hline $2329+398$ & $2332 \quad 13.7$ & $\begin{array}{lll}40 & 07 & 19\end{array}$ & & & 220 & 10 & 180 & 30 & 63 & 2 & 18 & 1 & 12 & 1 & $\mathrm{Q}$ \\
\hline $2330+387$ & 233302.6 & 390113 & 1350 & 78 & 1450 & 58 & 1120 & 45 & 915 & 27 & 457 & 9 & 252 & 5 & $\mathrm{~g}$ \\
\hline $2330+389$ & $2332 \quad 28.3$ & 391502 & 830 & 58 & 380 & 16 & 300 & 31 & 90 & 3 & 27 & 5 & 10 & 1 & \\
\hline $2330+397$ & 233256.2 & 400155 & & & 140 & 7 & 180 & 30 & 69 & 2 & 29 & 1 & 13 & 1 & \\
\hline $2330+402$ & 233253.0 & 403036 & 2020 & 109 & 2190 & 88 & 1780 & 61 & 825 & 25 & 338 & 7 & 150 & 3 & \\
\hline $2330+435$ & 233322.3 & $4346 \quad 47$ & 3320 & 171 & 1640 & 66 & 1150 & 46 & 309 & 9 & 56 & 2 & 16 & 1 & $\mathrm{~g}$ \\
\hline $2331+399$ & 233344.2 & 401228 & 1500 & 85 & 1010 & 41 & 830 & 39 & 293 & 9 & 93 & 2 & 42 & 1 & $\mathrm{G}$ \\
\hline $2332+388$ & 233502.4 & 390610 & 1100 & 68 & 540 & 22 & 420 & 33 & 136 & 4 & 46 & 1 & 19 & 1 & Q \\
\hline $2332+399$ & 233434.3 & 401322 & 430 & 45 & 460 & 19 & 390 & 32 & 166 & 5 & 62 & 2 & 23 & 1 & \\
\hline $2333+397$ & $23 \quad 35 \quad 28.9$ & $4000 \quad 09$ & & & 140 & 7 & 160 & 30 & 51 & 3 & 17 & 1 & 4 & 1 & \\
\hline $2333+399$ & 233533.7 & 401143 & & & 140 & 7 & 120 & 30 & 51 & 2 & 15 & 1 & 11 & 1 & \\
\hline $2334+398$ & 233655.7 & 400545 & 2340 & 124 & 1280 & 51 & 1010 & 43 & 480 & 14 & 160 & 3 & 45 & 1 & $\mathrm{~g}$ \\
\hline $2335+392$ & $23 \quad 38 \quad 11.3$ & 393333 & 1520 & 86 & 780 & 32 & 560 & 34 & 201 & 6 & 90 & 2 & 32 & 1 & $\stackrel{\circ}{Q}$ \\
\hline $2336+381$ & 233853.5 & 382324 & 2420 & 127 & 1240 & 50 & 900 & 40 & 327 & 10 & 103 & 2 & 42 & 1 & \\
\hline $2337+398$ & 233943.5 & $4008 \quad 45$ & 380 & 44 & 290 & 12 & 240 & 31 & 100 & 3 & 44 & 5 & 26 & 1 & $\mathrm{~g}$ \\
\hline $2338+390$ & $2341 \quad 13.9$ & 391829 & 1380 & 80 & 750 & 30 & 620 & 35 & 215 & 6 & 80 & 2 & 40 & 1 & $\mathrm{Q}$ \\
\hline $2338+393$ & 234121.0 & 393604 & 480 & 47 & 380 & 16 & 230 & 31 & 159 & 5 & 46 & 5 & 35 & 1 & \\
\hline $2340+386$ & $23 \quad 42 \quad 29.6$ & 385446 & 2510 & 132 & 1180 & 47 & 900 & 40 & 257 & 8 & 65 & 2 & 20 & 1 & \\
\hline $2341+396 \mathrm{~A}$ & 234407.1 & 395354 & & & 300 & 13 & & & 123 & 4 & 54 & 2 & 20 & 1 & $\mathrm{~g}$ \\
\hline $2341+396 \mathrm{~B}$ & $2344 \quad 13.8$ & 395205 & & & 260 & 11 & & & 98 & 4 & & & 23 & 1 & $\mathrm{~g}$ \\
\hline $2341+399$ & 234424.1 & 401251 & 890 & 60 & 440 & 18 & 350 & 32 & 139 & 4 & 55 & 2 & 34 & 1 & \\
\hline $2342+394$ & 234459.0 & 394626 & 400 & 45 & 150 & 8 & 110 & 30 & 29 & 2 & 7 & 1 & 2 & 1 & \\
\hline
\end{tabular}


Table 2. continued

\begin{tabular}{|c|c|c|c|c|c|c|c|c|c|c|c|c|c|c|c|}
\hline B3 name & $\begin{array}{c}\mathrm{RA}(\mathrm{J} 2000) \\
{\left[\begin{array}{ccc}\mathrm{h} & \mathrm{m} & \mathrm{s}\end{array}\right]}\end{array}$ & 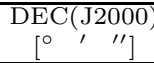 & $\begin{array}{r}S_{151} \\
{[\mathrm{mJy}]}\end{array}$ & $\begin{array}{r}\Delta S_{151} \\
{[\mathrm{mJy}]}\end{array}$ & $\begin{array}{r}S_{327} \\
{[\mathrm{mJy}]}\end{array}$ & $\begin{array}{r}S_{327} \\
{[\mathrm{mJy}]}\end{array}$ & $\begin{array}{r}S_{408} \\
{[\mathrm{mJy}]}\end{array}$ & $\begin{array}{r}\Delta S_{408} \\
{[\mathrm{mJy}]}\end{array}$ & $\begin{array}{l}S_{1400} \\
{[\mathrm{mJy}]}\end{array}$ & $\begin{array}{r}\Delta S_{1400} \\
{[\mathrm{mJy}]}\end{array}$ & $\begin{array}{l}S_{4850} \\
{[\mathrm{mJy}]}\end{array}$ & $\begin{array}{r}\Delta S_{4850} \\
{[\mathrm{mJy}]}\end{array}$ & $\begin{array}{r}S_{10550} \\
{[\mathrm{mJy}]}\end{array}$ & $\begin{array}{r}\Delta S_{10550} \\
{[\mathrm{mJy}]}\end{array}$ & Id. \\
\hline $2344+429$ & \begin{tabular}{|llll}
23 & 47 & 22.9
\end{tabular} & 431052 & 1820 & 99 & 1220 & 49 & 920 & 41 & 567 & 17 & 284 & 6 & 254 & 5 & $\mathrm{Q}$ \\
\hline $2347+397$ & $23 \quad 5025.2$ & 400202 & & & 120 & 7 & 120 & 30 & 31 & 1 & 8 & 1 & 4 & 1 & \\
\hline $2348+387$ & $2350 \quad 34.4$ & 390421 & 1090 & 68 & 530 & 22 & 430 & 33 & 164 & 5 & 43 & 1 & 21 & 1 & \\
\hline $2348+450$ & 235127.9 & $4518 \quad 30$ & 3280 & 169 & 1910 & 77 & 1800 & 62 & 744 & 22 & 226 & 5 & 88 & 2 & G \\
\hline $2349+396$ & 235203.3 & 395502 & 940 & 62 & 640 & 26 & 510 & 34 & 177 & 5 & 54 & 2 & 22 & 1 & \\
\hline $2349+410$ & 235152.9 & 412114 & 3200 & 165 & 1740 & 70 & 1380 & 51 & 454 & 14 & 115 & 3 & 48 & 1 & $\mathrm{Q}$ \\
\hline $2350+395$ & 235248.9 & 394754 & & & 280 & 12 & 150 & 30 & 199 & 6 & 197 & 4 & 262 & 5 & \\
\hline $2351+394$ & 235341.2 & 394323 & 1500 & 85 & 780 & 32 & 590 & 35 & 186 & 6 & 55 & 2 & 20 & 1 & \\
\hline $2351+398$ & 235341.0 & 400542 & & & 90 & 6 & 110 & 30 & 27 & 1 & 5 & 1 & 2 & 1 & \\
\hline $2351+400 B$ & 235357.2 & 401755 & 4350 & 221 & 2730 & 109 & 1980 & 67 & 828 & 25 & 268 & 29 & 158 & 3 & \\
\hline $2351+456$ & 235421.7 & 455304 & 2360 & 125 & 2230 & 89 & 2210 & 73 & 1878 & 56 & 1281 & 26 & 727 & 15 & $\mathrm{Q}$ \\
\hline $2352+385$ & 235438.0 & 384735 & 850 & 58 & 570 & 23 & 470 & 33 & 187 & 6 & 74 & 2 & 33 & 1 & \\
\hline $2354+397$ & 235707.3 & 400302 & 480 & 47 & 270 & 12 & 200 & 31 & 78 & 2 & 23 & 4 & 10 & 1 & \\
\hline $2354+471$ & 235730.6 & 472606 & 7660 & 385 & 5150 & 206 & 4400 & 135 & 1811 & 54 & 664 & 67 & 384 & 8 & $\mathrm{~g}$ \\
\hline $2355+397$ & 235811.7 & 400153 & & & 380 & 16 & 330 & 32 & 107 & 3 & 36 & 5 & 13 & 1 & \\
\hline $2355+398$ & 235801.0 & 400629 & 1180 & 71 & 660 & 27 & 560 & 34 & 199 & 6 & 65 & 2 & 27 & 1 & \\
\hline $2356+390$ & 235859.7 & $\begin{array}{lll}39 & 22 & 29\end{array}$ & 2040 & 110 & 1210 & 49 & 930 & 41 & 424 & 13 & 259 & 5 & 241 & 5 & Q \\
\hline $2356+437$ & 235836.0 & 440448 & 11700 & 586 & 6900 & 276 & 5360 & 164 & 1984 & 60 & 474 & 10 & 186 & 4 & G \\
\hline $2357+398$ & 235938.7 & 400636 & 710 & 53 & 380 & 16 & 320 & 31 & 91 & 3 & 40 & 5 & 11 & 1 & $\mathrm{~g}$ \\
\hline $2358+390$ & $\begin{array}{llll}00 & 00 & 41.5\end{array}$ & 391804 & 340 & 43 & 440 & 18 & 370 & 32 & 211 & 6 & 145 & 3 & 110 & 2 & \\
\hline $2358+406$ & $\begin{array}{llll}00 & 00 & 53.1\end{array}$ & 405401 & 1700 & 94 & 2060 & 83 & 2020 & 68 & 1306 & 39 & 517 & 10 & 260 & 5 & \\
\hline $2358+416$ & $\begin{array}{llll}00 & 01 & 19.6\end{array}$ & $\begin{array}{lll}4 & 5307\end{array}$ & 6610 & 333 & 2940 & 118 & 2210 & 73 & 554 & 17 & 103 & 10 & 31 & 1 & $\mathrm{G}$ \\
\hline $2359+394$ & $\begin{array}{lll}00 & 01 & 43.2\end{array}$ & 394621 & 2400 & 126 & 1320 & 53 & 960 & 42 & 366 & 11 & 104 & 2 & 43 & 1 & \\
\hline
\end{tabular}

